Portland State University

PDXScholar

Spring 6-1-2016

\title{
An Examination of School Readiness: How Is the Construct Defined for Children Who Are Blind?
}

Lisa Joann McConachie

Portland State University

Follow this and additional works at: https://pdxscholar.library.pdx.edu/open_access_etds

Part of the Educational Leadership Commons, and the Special Education and Teaching Commons Let us know how access to this document benefits you.

\section{Recommended Citation}

McConachie, Lisa Joann, "An Examination of School Readiness: How Is the Construct Defined for Children Who Are Blind?" (2016). Dissertations and Theses. Paper 2948.

https://doi.org/10.15760/etd.2943

This Dissertation is brought to you for free and open access. It has been accepted for inclusion in Dissertations and Theses by an authorized administrator of PDXScholar. Please contact us if we can make this document more accessible: pdxscholar@pdx.edu. 
An Examination of School Readiness:

How Is the Construct Defined for Children Who Are Blind?

by

Lisa Joann McConachie

A dissertation submitted in partial fulfillment of the requirements for the degree of

Doctor of Education

in

Educational Leadership: Special and Counselor Education

Dissertation Committee:

Sheldon Loman, Chair

Micki M. Caskey

Ruth Falco

Andrew Mashburn

Portland State University

2016 
C 2016 Lisa Joann McConachie 


\begin{abstract}
An increased focus on school readiness exists as students enter public school for the first time. Given unique challenges for students who are blind and use braille, school readiness in the transition to and preparation for kindergarten is even more critical to their future academic success. If children who are blind and use braille as their primary learning medium have the necessary school readiness skills entering kindergarten, they will be better prepared to participate in the academic classroom. There is a need for additional research to illuminate the skills needed for school readiness for children who are blind and use braille so programs can tailor their resources to provide appropriate instruction and intervention. The purpose of this research is to describe and explain how Teachers of the Visually Impaired (TVI) and primary caregivers construct the idea of school readiness for children who are blind and use braille as their primary learning medium. This research examined current practice in relation to the Head Start Early Learning Outcomes Framework and the Expanded Core Curriculum. Grounded in the Ecological and Dynamic Model of Transition the research situated the whole child within natural contexts to identify supports for successful transition to public school. Qualitative research design used permanent product review, including Individual Family Service Plans and Individual Education Plans to identify themes of current practice. Identified themes were verified through semi-structured interviews with key stakeholders to better understand school readiness for children who are blind.
\end{abstract}




\section{Dedication}

This work is dedicated to my loving family Todd, Grant and Ian McConachie. I am forever grateful for their unwavering support, love and encouragement. 


\section{Acknowledgements}

I would like to acknowledge the members of my committee for their time and support. I would also like to acknowledge Dr. Micki Caskey and Dr. Dannelle Stevens for their mentorship as cohort leaders and guides in the writing process. Finally, I would like to acknowledge Kate Panaccione and Vivian Garrison, I am forever grateful for their encouragement and friendship. 


\section{Table of Contents}

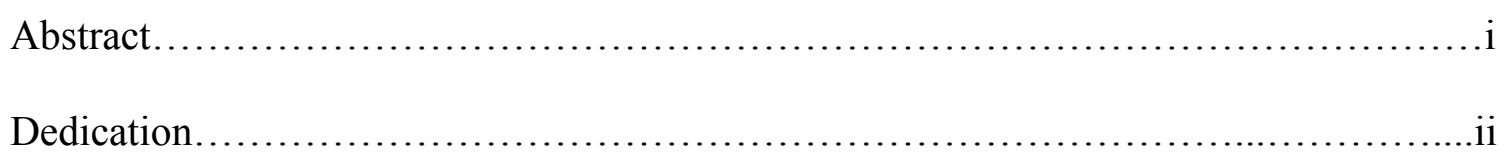

Acknowledgements............................................................

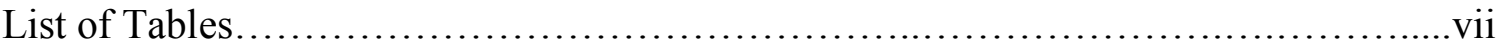

List of Figures...................................................................

Chapter 1 Problem Statement.....................................................

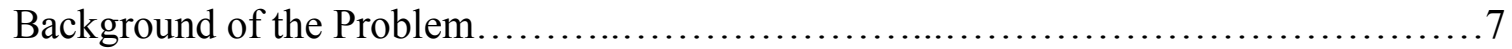

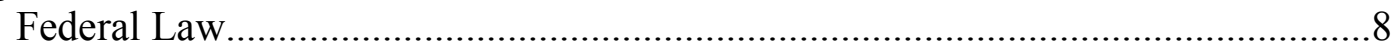

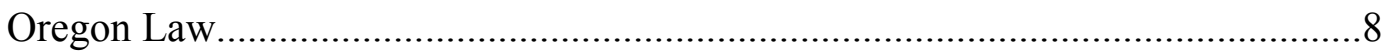

Oregon Low Incidence Disabilities..............................................................

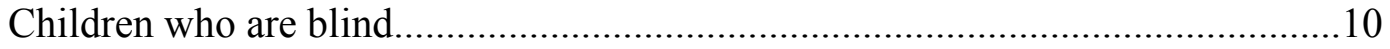

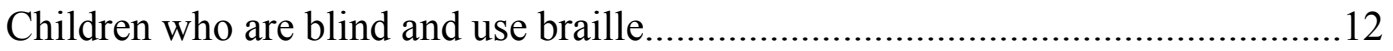

Special education services for children who are blind............................................13

Head Start Early Learning Outcomes Framework (USDHHS, 2015) .................14

Expanded Core Curriculum for blind and visually impaired.................................17

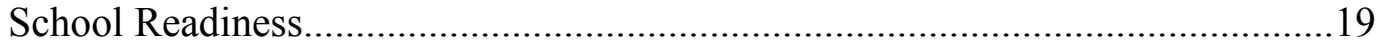

Statement of the Research Problem....................................................21

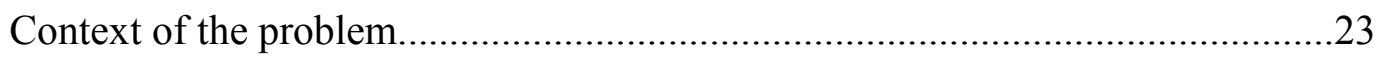

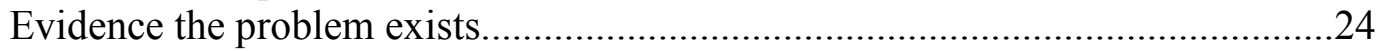

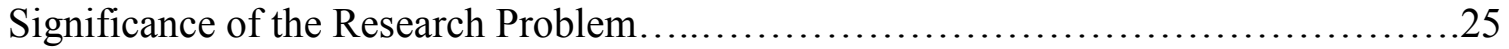

Presentation of Methods and Research Questions....................................26

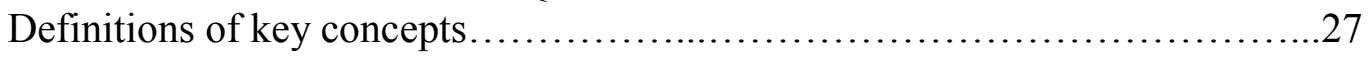

Chapter 2 Literature Review..................................................... 33

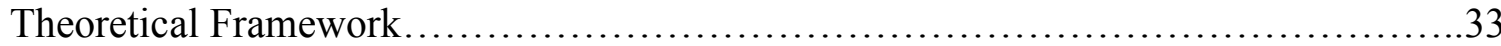

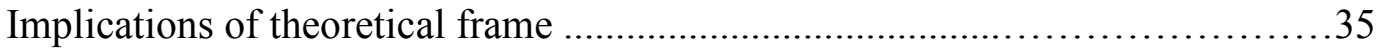

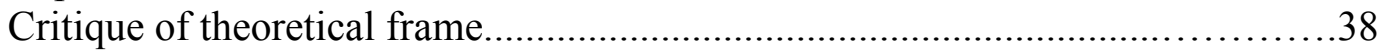

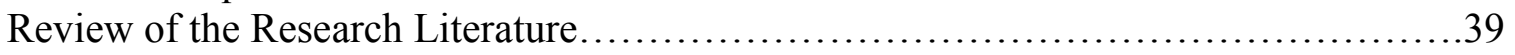

Developmental profile of children who are blind ..............................40

Historical perspective of school readiness..................................... 47

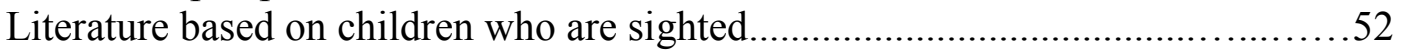


Ecological and Dynamic Model of Transition and readiness..........................58

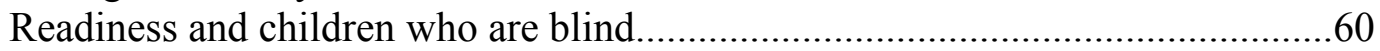

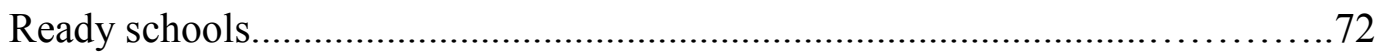

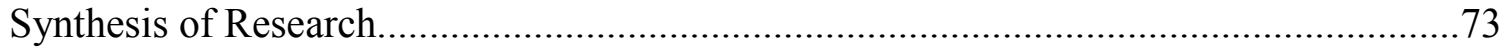

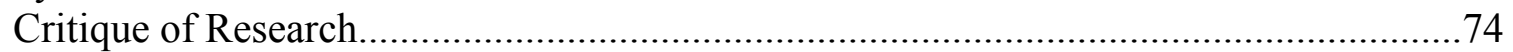

Review of the Methodological Literature............................................75

Justification of selection of methods........................................................

Summary of Research Literature and Application to Study..............................................77

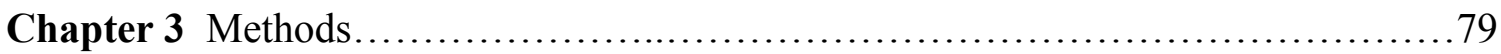

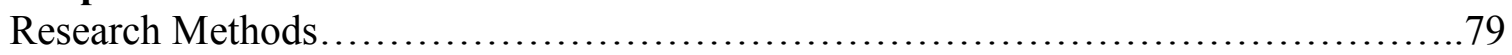

Type of research design and rationale ..................................... 81

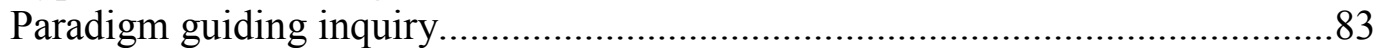

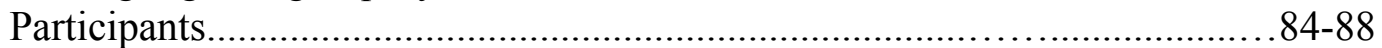

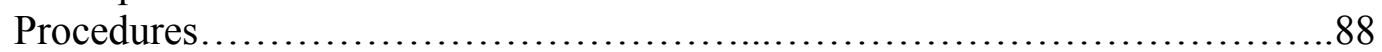

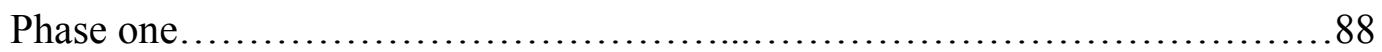

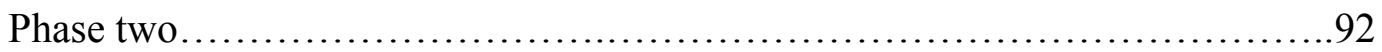

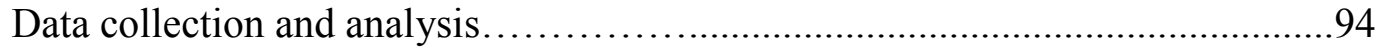

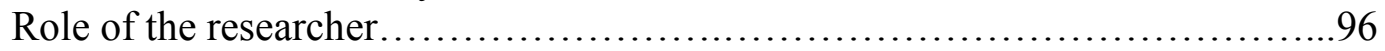

Chapter 4 Results/Analysis..................................................... 98

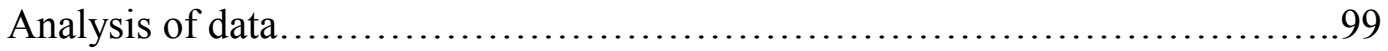

Presentation of results .................................................. 100

Interpretation of findings............................................... 125

Limitations of study ...................................................

Chapter 5 Discussion/Conclusion................................................. 134

Synthesis of findings..................................................

Situated in larger context............................................. 138

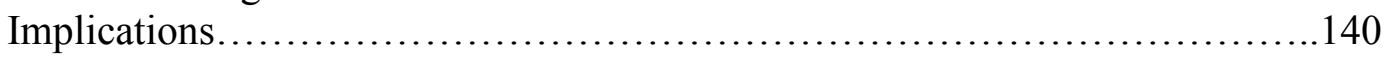

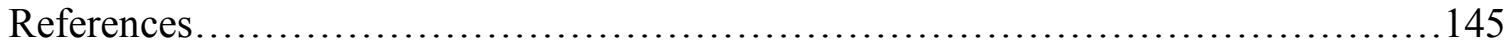

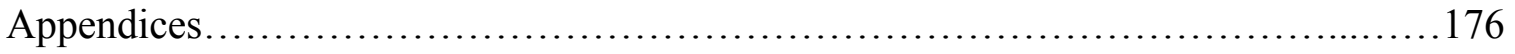

Appendix A. Request for documents..............................................................176 
Appendix B. Teachers of the visually impaired consent form................177

Appendix C. Primary caregivers consent form........................... 178

Appendix D. Sample questions for teachers of the visually impaired.......... 179

Appendix E. Sample questions for primary caregivers..................... 180

Appendix F. Student data analysis................................181-184 


\section{List of Tables}

Table

Page

1.1 Head Start Early Learning Outcomes Framework (USDHHS, 2015). $16-17$

1.2 Expanded Core Curriculum for Blind and Visually Impaired

(Sapp and Hatlen, 2010).

$18-19$

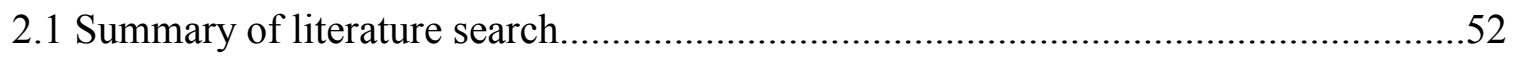

3.1 Summary of student participants .................................... $85-86$

3.2 Summary inter-observer reliability agreement: Head Start Early Learning Outcomes Framework

(USDHH2015). ...

3.3 Summary inter-observer reliability agreement: Expanded Core Curriculum (Hatlen, 1996).................................................91

3.4 Sample student data...................................................95

4.1 Alignment of IFSP and IEP goals and objectives with Head Start Early Learner Outcomes Framework (USDHHS, 2015) primary domains $\quad . . \ldots \ldots \ldots \ldots \ldots . . . \ldots 102$

4.2 Primary domain: Approaches to Learning with sub domains..................... 103

4.3 Primary domain: Social and Emotional Development with sub domains........... 104

4.4 Primary domain: Language and Communication with sub domains...............106

4.5 Primary domain: Literacy with sub domains .............................. 107

4.6 Primary domain: Mathematics with sub domains........................... 109

4.7 Primary domain: Scientific Reasoning with sub domains......................110

4.8 Primary domain: Perceptual, Motor, and Physical Development with sub domains......................................................... 111

4.9 Alignment of IFSP and IEP goals and objectives with Expanded Core Curriculum (Hatlen, 1996).................................................... 113 


\section{List of Figures}

Figure

Page

2.1 The Ecological and Dynamic Model of Transition

(Rimm-Kaufman \& Pianta, 2000).

4.1 Teacher of the Visually Impaired Service Time: Minutes per week...............115

4.2 Orientation \& Mobility Service Time: Minutes per week......................116 


\section{Chapter 1: Problem Statement}

Entering kindergarten is a milestone for families and children. This event marks

the beginning of a journey through $\mathrm{K}-12$ public schools. Beginning kindergarten is also a milestone for schools as they welcome a new incoming class, new students, and new families. "Kindergarten marks a child's entry into formal schooling, and performance in kindergarten paves the way for future academic success or failure" (Schulting, Malone, \& Dodge, 2005, p. 1). Kindergarten is a critical milestone for all students including those with identified disabilities, specifically blindness. Given the unique challenges for students who are blind and their families in K-12 educational agencies, school readiness in the transition to and preparation for kindergarten is even more critical to their future academic success as well as to the successful relationship between the family and public school (Daley, Munk, \& Carlson, 2011).

For all children, there is an increased focus on school readiness as children enter kindergarten; school readiness is broadly defined to include literacy, mathematical and cognitive skills, social, emotional, behavioral and physical development (Workman, Griffith, \& Atchison, 2014; Duncan et al., 2007; Farran, 2011; Kagan \& Rigby, 2003; Konold \& Pianta, 2005). School readiness, sometimes identified as kindergarten readiness, is a significant component of the transition to kindergarten. There is much debate regarding the definition of school readiness; however, what is agreed upon is the importance of children entering kindergarten with the skills to prepare them for public school and the academic environment (Duncan et al., 2007; Farran, 2011; Kagan \& Rigby, 2003; Kern \& Friedman, 2009; Konold \& Pianta, 2005; Raver \& Knitzer, 2002; Weiland \& Yoshikawa, 2013; Workman et al., 2014; Xue \& Meisels, 2004). School 
readiness is a term which generally focuses on "social and academic competencies that children are presumed to need to start school ready to learn" (Mashburn \& Pianta, 2006, p. 1). The concept of school readiness is significant in the field of disabilities as this measure historically was used to delay entry for students with disabilities or used as a reason for an alternative placement (Blyth \& Milner, 1994; Carlton \& Winsler, 1999; Gredler, 1992; May \& Campbell, 1981). In today's schools, chronological age is used as a requirement for school entry for children with and without disabilities. As age is the determinant factor for school entry, however, the national conversation of school readiness continues to focus on skill level. There is increased accountability and academic standards being pushed down toward preschool; school readiness is a critical aspect of a child's entry into kindergarten regardless of ability.

It is important that children who are blind and the significant adults in their educational experience are cognizant of the expectations for all children as they enter kindergarten. Children who are blind should have the same expectations for academic readiness and outcomes as their sighted peers. Children who are blind often lag behind their more typical peers with respect to cognitive and social-emotional skills even with services provided in early childhood special education (Bigelow, 1987; Erickson \& Hatton, 2007; Fraiberg, 1977; Preisler, 1995; Stratton \& Wright, 1991; Wormsley \& D’Andrea, 1997). Therefore, children who are blind entering kindergarten have additional and more complex needs than their sighted peers. Children who are blind entering kindergarten may not be prepared for the unfamiliar academic, behavioral and social demands and my be unsuccessful (Magnuson, Meyers, Ruhm \& Waldfogel, 2005; Rimm-Kaufman, Pianta, \& Cox, 2000; Schulting et al., 2005; Shore, 1998; Stormont, 
Beckner, Mitchell, \& Richter, 2005). Some researchers suggested lack of readiness is attributed in part to the dramatic differences in parent involvement, classroom organization, pedagogical approaches, and expectations of behavior and academic demands between early childhood settings and public school kindergarten classrooms (Early, Pianta, Taylor, \& Cox, 2001; Johnson, Gallagher, Cook, \& Wong, 1995; O’Brien, 1991; Schulting et al., 2005; Stormont et al., 2005). This is the case for children who are blind in addition to the complexity of concepts, academic and behavioral expectations and demands increase dramatically as they enter public school.

Educators report school readiness skills, a construct within the transition to kindergarten, as necessary for children to demonstrate as they enter school age services (Lin, Lawrence, \& Gorrell, 2003). School readiness is not a set of required skills for attendance in kindergarten. Readiness is not a way to prevent children with disabilities from attending general education; rather, readiness, involves a set of skills identified through research that support a child's successful transition into public school and creates a foundation for their later school success. However, when the classroom teacher, school administrators or primary caregivers perceive students who are blind as unprepared for kindergarten expectations, academic or behavioral, they are often placed in special education classrooms outside of the general education classroom away from typical peers and sometimes in residential schools for the blind if available in the state. This is a problem for school districts due to the high cost of self-contained classrooms and residential placements (Odom, Buysse, \& Soukakou, 2011) as well as the societal cost of isolating students with disabilities from their typical peers (Fitch, 2003). This is particularly a problem for children who are blind placed away from typical peers and 
needed language models (Andersen, Dunlea, \& Kekelis, 1984; Bigelow, 1987; Celeste, 2006; Warren, 1984).

The term "school readiness" is a legacy of the efforts of the National Education Goals Panel (NEGP) to improve the outcomes of our education system with the first goal being "All children in America will start school ready to learn" (National Education Goals Panel, 1995). There is evidence of the continuing focus on accountability and outcomes for students in preschool and their skills entering kindergarten. "The accumulation of convincing evidence from research [is] that young children are more capable learners than current practices reflect and that good educational experiences in the preschool years can have a positive impact on school learning" (Burns, Donovan, \& Bowman, 2000, p. 2). Early learning standards have been put into place to better define expectations for what children should know and be able to do prior to kindergarten (Scott-Little, Lesko, Martella, \& Milburn, 2007). In 2002, federal reforms and initiatives such as Good Start, Grow Smart, federal guidelines for childcare development and reauthorization of Head Start in 1998 have emphasized greater accountability for child outcomes (Scott-Little et al., 2007). Nationally, Race to the Top Grants for early intervention and early childhood services are increasing and States instituting universal preschool and increasing pre-kindergarten funding are on the rise (Workman et al., 2014). In Oregon, former Governor Kitzhaber's PreK-20 school initiative had a focus on the education system from preschool to grade three (Hammond, 2012; Johnson, 2015). In 2011, the Oregon Legislature under the direction of the former Governor called for a unified education system from preschool through college (Hammond, 2012; Johnson, 2015). Specific initiatives include the Kindergarten Partnership and Innovation grants 
and Early Literacy grants which included goals of increasing school readiness and early literacy skills, as measured in part by the Kindergarten Readiness Assessment. As part of the Governor's plan, the Early Learning Council was established to oversee the Early Learning System in the State. The Council was created in 2011 to guide efforts to streamline state programs and policy recommendations for early learning.

To address early learning for children with disabilities and receiving Early Intervention/Early Childhood Special Education (EI/ECSE) services, the Individuals with Disabilities Education Improvement Act (IDEIA) required State Interagency Coordinating Councils to advise EI/ECSE programs. "The State Interagency Coordinating Council (SICC) was established to ensure interagency coordination and to support the ongoing development of quality statewide services for young children and their families (By Authority of IDEA and ORS 343.499)" (Oregon House Bill 4165 Joint Workgroup, 2012). Through this partnership between the SICC and the Governor's appointed Early Learning Council, several recommendations were made regarding early childhood education services for children with identified disabilities. One of the key recommendations was to strengthen joint efforts through collaborative agreements between EI/ECSE providers and schools during a child's transition to kindergarten in addition to the creation of the Oregon Kindergarten Readiness Assessment (KRA) (Oregon House Bill 4165 Joint Workgroup, 2012). The KRA was developed to obtain information regarding early learning experiences for all children and to better understand the needs of students related to social-emotional development, self-regulation, and early literacy and math skills. 
While there is general agreement that school readiness is defined as social and academic competencies for all children, for children who are blind the definition is ambiguous. Researchers suggest that children who are blind lag behind their sighted peers in the areas of literacy, mathematical concepts, language and conceptual development (Bigelow, 1987; Erickson \& Hatton, 2007; Fraiberg, 1977; Preisler, 1995; Stratton \& Wright, 1991; Wormsley \& D'Andrea, 1997). Researchers have identified braille readiness checklists and strategies but there is no comprehensive school readiness research specifically for children who are blind (Lamb, 1996; MacComiskey, 1996). A defined and comprehensive set of readiness skills is needed to guide educators, so that children who are blind and entering kindergarten develop the necessary skills and attributes to access and participate in the general education curriculum. The purpose of this research is to describe and explain how teachers of the visually impaired (TVI) and primary caregivers construct the idea of kindergarten readiness for children who are blind, identified tactile learners, and use braille as their primary learning medium.

My hypothesis is the type and intensity of education services for children who are blind do not align with the current construct of school readiness for sighted children. Children who are blind are sometimes perceived by general education teachers as needing extensive supports to participate in general education and therefore, the general education classroom may not be seen as an appropriate education placement. If children who are blind have the necessary school readiness skills including academic and social skills when entering kindergarten, then they will be better prepared to participate fully in the academic classroom and placement in the general education classroom will be identified as the least restrictive placement. Children who are blind entering kindergarten have 
additional and more complex needs than their sighted peers. This study will explain how TVIs and primary caregivers are preparing children who are blind for kindergarten in general education classrooms.

\section{Background of the Problem}

To understand the importance and significance of research in the area of school readiness for children who are blind, it is important for the reader to understand the complex and extensive background of education of children who are blind. I review the federal and state law that guides special education for children with disabilities, both ages three to five and in public school. I discuss the unique educational service delivery model for students with low incidence disabilities including blindness in Oregon. I highlight the unique characteristics of children who are blind and specifically children who are blind and use braille as their primary learning medium. I include an overview of early learner standards, expanded core curriculum for children who are blind, and The Oregon Project for Visually Impaired and Blind Preschool Children: Skills Inventory (Brown, Simmons, \& Methvin, 1978), a commonly used skills inventory for preschool children who are blind. Lastly, I define kindergarten readiness, as I interpret the construct for the purposes of this research as well as what I mean when I refer to a child as blind.

When a child is blind and enters kindergarten, they have already been identified as a child with a disability and have received services under IDEIA through Early Childhood Special Education (ECSE). The following provides a summary of federal and state laws under which children receive services. 


\section{Federal law}

The IDEIA is the federal law that governs how public school districts and state agencies provide special education to students with disabilities. IDEIA is a reauthorization of original legislation entitled Education for All Handicapped Children Act, passed in 1975. IDEIA mandates that all public school districts receiving federal dollars be required to provide students with disabilities "a free and appropriate public education that emphasizes special education and related services designed to meet their unique needs and prepare them for further education, employment, and independent living" (USDOE, 2004). IDEIA defines a child with a disability as a child with one of the following eligibilities: Intellectual disability, hearing impairment (including deafness), speech or language impairments, visual impairments (including blindness), emotional disturbance, orthopedic impairments, autism, traumatic brain injury, other health impairments, or specific learning disabilities. Eligibility for special education requires identification in one of the disabilities areas and the need for specially designed instruction and related services. IDEIA is written in three parts: Part A includes the provisions of the law, Part B addresses children from age three through age 2, and Part C addresses children, birth through age two. This research will focus on IDEIA Part B, specifically children who are legally blind and tactile learners, age three to five receiving Early Childhood Special Education (ECSE) and their entry into K-12 public school.

\section{Oregon law}

In Oregon, early intervention/early childhood special education (EI/ECSE) programs have been established by Oregon Administrative Rules to close developmental delays due to the impact of a child's disability. Early intervention (EI) serves children, 
birth through age two; ECSE services children, age three through age five. Services are provided only to children with identified disabilities defined by IDEIA and/or significant delays and children who are born with a condition likely to result in a developmental dely. Once a child is identified as meeting the criteria of eligibility, each child receives an individualized service plan called an Individual Family Service Plan (IFSP) based on the child and family's unique needs. IFSP plans are intended to summarize all services needed by the family and child to meet the child's identified goals. ECSE services may include therapies, specialized educational supports and parent training provided in a variety of settings including through home visits, childcare programs, community preschools, and in specialized classrooms (Oregon House Bill 4165 Joint Workgroup, 2012). Currently the Oregon Department of Education (ODE) contracts with nine contractors to coordinate EI/ECSE programs throughout the state. Approximately 12,000 children received EI/ECSE services in Oregon, 177 of which were identified as being eligible under vision impairment (Oregon House Bill 4165 Joint Workgroup, 2012).

\section{Oregon low incidence disabilities}

In Oregon, in addition to EI/ECSE services and special education services provided by school districts, regional programs provide services for specific disabilities identified as low incidence serving students birth through age 21 (Special education provided by state through local, county or regional program, ORS 343.236, 2015). In 1985, the Oregon legislature created regional programs to provide equity of access to specialized services and staff to educate students with specific low incidence disabilities including autism spectrum disorder, vision impairment including blindness, deaf or hard of hearing, deaf-blindness, severe orthopedic impairments and traumatic brain injury. 
Regional programs serve multiple districts and EI/ECSE programs and are operated either by an Education Service District or school district. The primary responsibility for a child's education remains with the child's district of residence. Regional programs were designed to provide districts with services for students with specific disabilities that occur at such a low rate in the general population that it may be difficult for districts to hire specialized staff or have the unique resources to meet their needs. There are currently eight regional programs located in Oregon. In 2007, ODE asked American Institutes for Research to conduct an independent assessment and analysis of the fiscal and operational efficiency of the EI/ECSE and Regional Low Incidence Programs (Parrish \& Harr, 2007). The findings of the independent evaluation highlight the unique services in Oregon among school districts, regional programs and EI/ECSE providers for students who have low incidence disabilities including those who are blind.

\section{Children who are blind}

Blindness is a relatively rare, low incidence disability compared to the identified categories of IDEIA. Ed Data Express, the U.S. Department of Education website indicates that $12.9 \%$ of K-12 students have an identified disability (U.S. Department of Education, National Center for Education Statistics, 2015); identification of vision impairment was $2.4 \%$ of the total (Erickson \& Shrader, 2013). Under IDEIA, visual impairment is defined as, "Visual impairment including blindness means an impairment in vision, that even with correction, adversely affects a child's educational performance. The term includes both partial sight and blindness" (USDOE, 2004). The Oregon Administrative Rules for Special Education (2013) defined vision impairments as the following: 
The student's acuity is $20 / 70$ or less in the better eye with correction; or the student's visual field is restricted to 20 degrees or less in the better eye; or the student has an eye pathology or a progressive eye disease which is expected to reduce either acuity or visual field to either an acuity level of 20/70 in the better eye or a visual field of 20 degrees or less in the better eye; or the assessment results of a licensed ophthalmologist or optometrist are inconclusive, and the student demonstrates inadequate use of vision.

This research will focus on students who meet the eligibility criteria in Oregon and receive their educations services through regional programs.

In the United States, students identified as vision impaired under IDEIA are $2.4 \%$ of the total special education population (Erickson \& Shrader, 2013). In 1990, data on legal blindness indicated approximately 2,600 children under five years of age and approximately 51,000 between the ages of 5-19 were legally blind (Chiang, Bassi, \& Javitt, 1992). The American Printing House for the Blind (APH) collects data from every state to identify the population of legally blind individuals birth through age 21 . Based on the APH Annual Report 2014: Distribution of Eligible Students Based on the Federal Quota Census of January 7, 2013, there were a total of 10,167 children under five years of age and approximately 50,226 between the ages of 5-21 were legally blind (APH Annual Report, 2014). In January of 2014, 412 children birth through kindergarten age in the United States were identified as tactile learners with a primary learning medium of braille. The number of braille readers steadily increased from 177 to 220 in first grade to 293 in second grade. From third grade to eleventh grade, the number of braille readers ranged 287 to 340 which suggests that the learning medium may be difficult to determine 
until a child is older and exposed to more instruction. A significant increase in braille readers to 405 in Grade 12 is not addressed in this paper.

This research will focus on Teachers of the Visually Impaired (TVIs) working with students entering kindergarten who meet the Oregon eligibility criteria under vision impairment and whose vision loss is such that a determination has been made that the student is a tactile learner and braille will be the student's primary learning medium. By referring to a child who is blind I mean they have met the Oregon definition of blindness which is an acuity of 20/200 or less in the better eye with a correction or a field loss such that there is less than 20 degrees of vision, or a progressive eye disease that leads to either of these conditions in the future. In addition to legal blindness, the IFSP or IEP team has determined that the student is a tactile learner and braille is the primary learning medium for the student.

\section{Children who are blind and use braille}

IDEIA requires all IEP teams to annually consider special factors that may impact a child's participation and progress in general education. One of these considerations is for braille and states:

In the case of a child who is blind or visually impaired, provide for instruction in braille and the use of braille unless the IEP Team determines, after an evaluation of the child's reading and writing skills, needs, and appropriate reading and writing media (including an evaluation of the child's future needs for instruction in braille or the use of braille), that instruction in braille or the use of braille is not appropriate for the child. (USDOE, 2004, Sec. 300.324(a)(2)(iii)) 
The assessment most often used to determine the need for braille is the Learning Media Assessment (Koenig \& Holbrook, 1995). The Learning Media Assessment (LMA) is an accepted tool created by the educator based on professional guidelines but is not a research-based, valid and reliable assessment. For the purposes of this research a LMA is a tool to determine the student's primary learning medium or media and primary literary medium or media. The LMA addresses the efficiency with which the student gathers information from various sensory channels (visual, tactile, auditory), the types and general learning media (e.g. braille, print, enlarged print) the student uses or will use to accomplish learning tasks, and the literacy media the student will use for reading and writing. The LMA is important in this study as the LMA identifies how a student with vision impairment will access the general education curriculum. If the team determines the child is a tactile learner and braille is the primary learning medium, it is imperative the TVI, special and general education teachers, and parents begin to plan for the unique needs of a student who uses braille in the classroom. The APH Annual Report captures results of LMAs for all students birth through age 21 nationally: $8.5 \%$ of students were identified as braille readers, $29.2 \%$ print, $9.2 \%$ auditory, $34.8 \%$ non-readers, and $18.3 \%$ pre-readers (APH Annual Report, 2014).

\section{Special education services for children who are blind}

In the United States, the nature and intensity of special education for children who are blind varies from state to state. There are currently 33 residential schools in the United States for students who are blind serving about $8.5 \%$ of the visually impaired population (APH Annual Report, 2014). The majority of students (83\%) who have vision 
impairment or are blind receive educational services through a combination of their public schools and regionalized services (APH Annual Report, 2014).

In 2010, the Oregon legislature closed the School for the Blind due to low enrollment (at the time of the closure there were 28 students ages 14 to 21). In Oregon, TVIs and/or Orientation and Mobility Specialists depending on the needs of the student provide special education services. Orientation and Mobility Specialists are educators who teach individual with vision impairment and blindness to travel independently and safely in their school environment, home, and community. Specially designed instruction, including type and intensity, is determined by the IEP team and reviewed on an annual basis. Students in Oregon are served in their local school districts and supported through the low incidence regional programs previously described.

Each State in the United States designs their unique services for students who are blind. Oregon established eight regional programs to serve students. Licensure requirements vary by state, however, Oregon requires teachers be certified as TVIs, including Orientation and Mobility Specialists (they must be dual-certified in both areas). Services are provided consistently throughout the state due to the collaborative nature of the regional programs and services. Unique to Oregon is that children, age three to five, are provided services though early childhood service providers not local school districts. Services are determined by the IFSP team and designed around the family needs as well as unique needs of the child. All services for students, age 3-21, are funded through IDEA Part B funds and include both federal and state funding.

\section{The Head Start Early Learning Outcomes Framework}


Standards-based education in the areas of literacy, language, and mathematics in pre-kindergarten is a critical part of a state's system of education service delivery (Neuman \& Roskos, 2005). States are identifying the need for children to have foundational knowledge as they enter kindergarten that aligns with common core K-12 standards; all 50 states have identified early learning standards for children, age three to five. Burns, Donovan, and Bowman (2001) suggested "The accumulation of convincing evidence from research [is] that young children are more capable learners than current practices reflect and that good educational experiences in the preschool years can have a positive impact on school learning” (p. 2). Researchers suggest young children are capable of learning more in the area of academic skills than many have thought previously and children's experiences before they start school are vitally important in shaping how successful children are later in their academic careers (Burns et al., 2001; Carlton \& Winsler, 1999; Daley et al., 2011; Duncan et al., 2007; Farran, 2011; Johnson et al., 1995; Kagan \& Rigby, 2003; Kern \& Friedman, 2009; Schulting et al., 2005; Weiland \& Yoshikawa, 2013). Early childhood educators are increasingly held accountable for measurable outcomes in domains that include academic, social emotional and behavior expectations. Early learning standards have been established, in part, to better define expectations for what children should know and be able to do prior to kindergarten (Neuman \& Roskos, 2005; Scott-Little, Kagan, \& Frelow, 2006; Scott-Little et al., 2007).

Oregon has adopted the Head Start Early Learning Outcomes Framework (U.S. Department of Health and Human Services (USDHHS, 2015) as the early learning standards for children, age three to five. The framework is intended to guide Head Start 
programs serving three to five-year old children on key elements of school readiness.

Additionally, the intention is to facilitate shared understanding of expectations across

persons educating young children including early childhood teachers, caregivers and

kindergarten teachers. Shared understanding can contribute to a more coherent approach

to educating young children and increasing a child's preparedness and success in early

elementary school and beyond (Hyson \& Biggar, 2006; Scott-Little et al., 2006). Table

1.1 is a summary of the Head Start Early Learning Outcomes Framework (USDHHS, 2015).

Table 1.1

Head Start Early Learning Outcomes Framework (USDHHS, 2015)

\begin{tabular}{|c|c|}
\hline $\begin{array}{c}\text { Primary Domains: Sub-domains } \\
\text { (preschool) }\end{array}$ & Description \\
\hline $\begin{array}{l}\text { Approaches to learning: } \\
\text { Emotional \& behavioral self-regulation } \\
\text { Cognitive self-regulation (executive } \\
\text { functioning) } \\
\text { Initiative \& curiosity } \\
\text { Creativity }\end{array}$ & $\begin{array}{l}\text { Incorporates emotional, behavioral, and cognitive } \\
\text { self-regulation. Includes initiative, curiosity, and } \\
\text { creativity. Navigate learning experiences including } \\
\text { challenges, frustration or those that take time to } \\
\text { accomplish. Includes self-regulation known as } \\
\text { executive functioning. Includes sustained attention, } \\
\text { impulse control, and flexibility in thinking. Related } \\
\text { skills are working memory, the ability to hold } \\
\text { information and manipulate it to perform tasks. }\end{array}$ \\
\hline $\begin{array}{l}\text { Social \& emotional development: } \\
\text { Relationship with adults } \\
\text { Relationship with other children } \\
\text { Emotional functioning } \\
\text { Sense of identity and belonging }\end{array}$ & $\begin{array}{l}\text { Ability to create and sustain meaningful } \\
\text { relationships with adults and children including } \\
\text { problem solving skills, forming relationships with } \\
\text { peers. Critical social skills, such as compromise, } \\
\text { cooperation, and sharing. To express, recognize, } \\
\text { and manage their own emotions and respond to } \\
\text { others' emotions. }\end{array}$ \\
\hline
\end{tabular}

(Continued) 
(Continued)

\begin{tabular}{ll}
\hline $\begin{array}{l}\text { Primary Domains: Sub-domains } \\
\text { (preschool) }\end{array}$ & Description \\
\hline Literacy: & $\begin{array}{l}\text { Refers to beginning to understand how the written } \\
\text { language is structured into sounds and symbols. }\end{array}$ \\
Phonological awareness & Understand rhyming and learn names of letters and \\
Print and alphabet knowledge & associated sounds. Recognizing name and practice \\
Comprehension and text structure & writing. Beginning to understand conventions and \\
Writing & functions of print (books, grocery lists, etc.). Begin \\
& to understand storybooks and retell or enact events \\
& understanding sequence, character development and \\
& casual relationships.
\end{tabular}

Cognition: Mathematics development:

Counting and cardinality

Operations and algebraic thinking

Measurement

Geometry and spatial sense

Cognition: Scientific reasoning:

Scientific inquiry

Reasoning and problem-solving
Refers to understanding numbers and quantities, their relationships and operations including add and subtracting quantities. Also includes knowledge of shapes, measurement, reasoning, classification and patterns.

Refers to the ability to develop knowledge about natural and physical world, learning scientific methods, reasoning, and problem solving skills. Included is the process of learning how things work, to use measurement, observation and tools. Includes learning vocabulary, fostering a sense of curiosity and motivation to learn.

Represents four elements: perception, gross motor, fine motor and health, safety, and nutrition.
Perceptual, Motor, and Physical development:

Gross motor

Fine motor

Health, safety, and nutrition

Expanded core curriculum. The notion that children who are blind have additional skills and knowledge needs beyond the core curriculum has been a topic discussed by professionals for many years (Hatlen, 1996). The concept has been identified by many names such as specialized curriculum, disability specific skills, nonacademic curriculum and most recently the Expanded Core Curriculum (ECC) (Hatlen, 1996). The ECC does not replace the traditional core curriculum; it identifies needed skills and knowledge in addition to the core curriculum unique to children who are blind. There are nine areas identified in the ECC intended to identify the skills and 
knowledge a blind child needs to learn to access and succeed in the core curriculum. The ECC is identified as a curriculum taught by TVIs who are trained in these unique skills and instructional strategies. The ECC is necessary for children who are blind due to the unique nature of their disability. Many skills included in the ECC are skills and knowledge that children who are sighted learn incidentally by observing others, understanding and interpreting nonverbal information and modeling adults and/or peers in the typical environment. Table 1.2 is a summary of the nine areas of the ECC.

\section{Table 1.2}

Expanded Core Curriculum for Blind and Visually Impaired (Sapp \& Hatlen, 2010)

\begin{tabular}{|c|c|}
\hline Expanded Core Curriculum Area & Definition \\
\hline Compensatory or access skills & $\begin{array}{l}\text { Refers to concept development, skills in } \\
\text { organizational, speaking and listening, and } \\
\text { accommodations including braille, optical devices, } \\
\text { digital access and tactile symbols }\end{array}$ \\
\hline Career Education & $\begin{array}{l}\text { Children who are sighted learn vocational } \\
\text { opportunities and work habits through visual } \\
\text { observation; those who are blind do not and require } \\
\text { specialized and direct instruction. }\end{array}$ \\
\hline Independent Living Skills & $\begin{array}{l}\text { Includes personal hygiene, food preparation, } \\
\text { financial management and organizational skills. }\end{array}$ \\
\hline Orientation and Mobility & $\begin{array}{l}\text { A systematic method to teach blind and visually } \\
\text { impaired children to travel in their environments } \\
\text { including school, home, neighborhood and } \\
\text { community. }\end{array}$ \\
\hline Recreation and Leisure & $\begin{array}{l}\text { Includes specific sports and activities designed for } \\
\text { blind individuals as well as learning skills of typical } \\
\text { sports and activities. }\end{array}$ \\
\hline Social Interaction Skills & $\begin{array}{l}\text { Observing peers or adults involved in social } \\
\text { interactions within natural environments supports } \\
\text { the learning of social skills. Children who are blind } \\
\text { are unable to access incidental learning of social } \\
\text { skills and norms. Nuanced social skills require } \\
\text { direct instruction. }\end{array}$ \\
\hline
\end{tabular}


(Continued)

Expanded Core Curriculum Area

Self Determination

Assistive Technology

Sensory Efficiency
Definition

Refers to the process by which a person controls their own life, makes their own decisions and choices without undue influences.

Refers to technology to support learning and access to the general education curriculum. Includes universal technology such as computers, tablets and mobile devices as well as specialized technology including optical and magnification devices, braille displays and embossers and specialized mobility devices.

Refers to the use of residual vision as well as using other senses to gain information from the environment or to access curriculum.

School readiness. Researchers agree children enter kindergarten at different ability levels and levels of preparedness due to varying early childhood experiences, unique developmental patterns and childhood experiences prior to the kindergarten year (Hatcher, Nuner, \& Paulsel, 2012; Johnson et al., 1995; Lin et al., 2003; Stormont et al., 2005). Framing the challenges of students who are blind in the construct of school readiness, allows educators to situate the child's needs in a context similar to their sighted peers. This research will focus on the construct of school readiness as defined in the literature for sighted children. While this research will also highlight the ECC and area of needs, research to highlight the challenges children who are blind face as they enter typical kindergartens will provide resources and guidance so programs can tailor their instruction and supports to increase a blind child's success in the typical kindergarten classroom.

The definitions of school readiness have evolved over time. As previously stated, initially readiness skills were initially determined to include the following: physical and 
motor development, social and emotional development, approaches toward learning (i.e., creativity, initiative, attitudes, toward learning, task mastery), language, cognition and, general knowledge (Kagan, Moore, \& Bredekamp, 1995; Meisels, 1999). During the last two decades focus on readiness skills began to increasingly incorporate academic-focused skills (Neuman \& Roskos, 2005). During this period, there was a national policy shift to accountability systems for learning early literacy, language, and numeracy for early childhood educators including Head Start (Neuman \& Roskos, 2005). Emphasis on academic readiness represented a significant departure for early childhood programs that historically focused on the belief that being healthy and well-adjusted was as important to early development as learning to read (Meisels, 1999). The national conversation shifted from children starting school ready to learn to children entering kindergarten with measurable academic skills in the area of literacy and numeracy in addition to social emotional and behavioral expectations (Hatcher et al., 2012).

The shift in federal and state education policies to emphasize children's academic readiness was driven in part from research suggesting many children with and without identified disabilities enter kindergarten ill-prepared for academic and behavioral requirements expected in elementary school. In a national survey of more than 3,000 kindergarten teachers, Rimm-Kaufman, Pianta, and Cox (2000) found that 46\% of teachers reported that about half or more of their class were unable to follow directions when they entered school. Following directions was a primary concern for kindergarten teachers in addition to other difficulties including children's lack of academic skills, a disorganized home environment, difficulty in working independently, lack of any formal preschool experience, and difficulty in working as part of a group, among others (Rimm- 
Kaufman et al., 2000). Research to align school readiness for children who are blind to the current national conversation is needed to ensure children who are blind are receiving the appropriate amount of instruction focused on those skills that will prepare them for public school.

In this research, school readiness is defined as a child who is blind and a tactile learner and, has progress in all areas of the child development and early learning as outlined by the Head Start Early Learning Outcomes Framework (USDHHS, 2015). The framework is intended to outline essential areas of development and learning to "establish school readiness goals for children, monitor children's progress, align curricula, and conduct program planning" (USDHHS, 2015, p. 2). Children who are ready for school, demonstrate skills and development in the following domains: Approaches to Learning, Social and Emotional Development, Language and Communication, Literacy, Cognition including Mathematics Development and Scientific Reasoning, and Perceptual, Motor, and Physical Development. Skill development and process should be reflected in the present levels of development, goals and objectives identified by the IFSP team between the child's ages three to five. School readiness is not a set of required skills for attendance in kindergarten. Readiness is not a way to prevent children with disabilities from attending general education, rather, readiness involves a set of skills identified through research that support a child's successful transition into public school and creates a foundation for their later school success

\section{Statement of the Research Problem}

Children who are blind and entering kindergarten have additional and more complex needs than their sighted peers (Fraiberg, 1977; Lowenfeld, 1964; Warren, 1984). 
Given the current emphasis on kindergarten readiness for all children, outlining skills that will help make children who are blind "ready" for kindergarten is essential. The purpose of this research is to describe and explain how TVIs and primary caregivers understand and define the construct of school readiness for children who are blind, identified tactile learners and use braille as their primary learning medium. My hypothesis is that the types and intensity of preschool education services, for children who are blind, do not align with the current idea of school readiness for sighted children.

Children who are blind may be perceived by general education teachers as needing extensive supports to participate in general education; and, therefore, the general education classroom may not be seen as an appropriate education placement. If children who are blind have the necessary school readiness skills, including academic and social skills when entering kindergarten, then they will be better prepared to participate fully in the academic classroom and placement in the general education classroom. Children who are blind and entering kindergarten have additional and more complex needs than their sighted peers. To test the hypothesis that the type and frequency of instruction may not align with expectations for sighted children, I developed a primary research question and supporting questions. Using the Head Start Early Learning Outcomes Framework (USDHHS, 2015) and the Expanded Core Curriculum (Hatlen, 1996) as foundations for school readiness, this research will describe and explain how TVIs and primary caregivers construct the idea of school readiness for children who are blind and use braille as their primary learning medium. The primary research question guiding this study is: How is the construct of school readiness defined for children who are blind and use braille as their primary medium? Additional questions examined include: (a) What is 
the current focus of instruction for children who are blind in preschool to prepare them for kindergarten? (b) What are the roles of the TVI and primary caregiver in preparing students for kindergarten? (c) What do TVIs and primary caregivers identify as the barriers to school readiness for children who are blind? (d) What do TVIs and primary caregivers see as essential to preparing children who are blind for kindergarten?

Children who are blind often lag behind typical peers with respect to cognitive and social-emotional skills even with services provided in ECSE (Bigelow, 1987; Erickson \& Hatton, 2007; Fraiberg, 1977; Preisler, 1995; Stratton \& Wright, 1991; Wormsley \& D'Andrea, 1997). Early gains for students with disabilities, including those who are blind, who have been schooled prior to kindergarten, especially in the area of pre-academic skills, fade as students transition to kindergarten and move through the early grades (Magnuson et al., 2005; Shore, 1998). Children who are blind entering kindergarten are often not prepared and can experience challenges when they encounter unfamiliar academic demands, rules, routines, atmosphere or relationships that differ dramatically between preschool settings and general education classrooms (Magnuson et al., 2005; Rimm-Kaufman \& Pianta, 2000; Schulting et al., 2005; Shore, 1998; Stormont et al., 2005). Therefore, there is a need for additional research to illuminate the skills needed for school readiness so that programs can tailor their resources to provide appropriate instruction and intervention.

\section{Context of the Problem}

This research will focus on TVIs and primary caregivers of children who are blind, identified as tactile learners, and use braille as their primary learning medium. Participants will be TVIs and primary caregivers of preschool or school age children who 
SCHOOL READINESS: CHILDREN WHO ARE BLIND

are receiving their educational services in Oregon. Student data sets will include IFSP and IEPs developed by educational teams from preschool years through kindergarten.

\section{Evidence that Problem Exits}

In my experience, children who are blind and enter kindergarten can be a source of "fear of the unknown" for teachers, including both general and special education professionals. Due to the relative rarity of students who are blind, the assumption may be the child should attend a school for the blind or at a minimum a specialized classroom (U.S. Department of Education, National Center for Education Statistics, 2015). There can be a general fear of safety for the child and an uncertainty of how to teach a child with such specialized needs. Due to these unique circumstances, there can be significant concerns regarding how to meet a child's educational needs in the general education classroom.

As previously stated, school readiness skills are increasingly a topic of conversation among policy makers and educators in Oregon. Research suggests the skills students have as they enter kindergarten have an effect far into their elementary and secondary schooling (Bailey, 2014; Duncan et al., 2007; Farran, 2011; Goldstein, Warde, \& Peluso, 2013; Kagan \& Rigby, 2003; Kern \& Friedman, 2009; Konold \& Pianta, 2005; Magnuson, Ruhm, \& Waldfogel, 2007; Ramey \& Ramey, 2004). Children who are blind are typically developmentally behind their sighted peers (Warren, 1984; Warren \& Hatton, 2003). It is critical for a student who is blind that school readiness is a focus earlier in their education to mitigate delays and provide students with the necessary skills and concepts to participate with their sighted peers in the areas of social and academic domains. 
SCHOOL READINESS: CHILDREN WHO ARE BLIND

Significance of the Problem

In this section, I highlight the educational significance of this research. The purpose of this research is to describe and explain how TVIs and primary caregivers construct the idea of school readiness for children who are blind and use braille as their primary learning medium. I examine current research regarding school readiness for sighted children and children who are blind and identify for the reader how my research will add to the body of knowledge of instruction for children who are blind and use braille as their primary learning medium.

Students entering kindergarten with a primary learning medium of braille are relatively rare; however, their unique disability and resulting needs require intensive specially designed intervention to ensure academic progress in the general elementary school setting. The problem is children who are blind entering kindergarten have additional and more complex needs than their sighted peers. A lack of school readiness can significantly and negatively impact a child's academic transition and progress (Daley et al., 2011; Mashburn \& Pianta, 2006; Schulting et al., 2005). If TVIs have misconceptions or misidentify concepts and skills needed for school readiness, then adequate instruction may not be provided in preschool years. If the academic and behavior expectations differ dramatically from preschool to kindergarten, children who are blind are at risk of losing skills gained in the preschool setting with a potential for placement outside of the general education classroom.

Exploring the topic of school readiness for children who are blind, are tactile learners and use braille could potentially increase the effectiveness of instruction and services from TVIs. This research will seek to identify how TVIs and primary caregivers 
define the construct of school readiness skills for children who use braille as their primary medium. This research will potentially identify alignment between school readiness for all children and those who are identified braille readers. Results of this research could potentially inform current practice to align with common core state standards and the changing landscape of kindergarten curriculum. This research will add to the body of research regarding primary caregivers' understanding of blindness and the resulting impact on expectations for development.

\section{Presentation of Methods and Research Question}

In the following section, I identify methods I used to answer questions relevant to identification of school readiness skills for children who are blind. The problem is children who are blind entering kindergarten have additional and more complex needs than their sighted peers. The purpose of this research is to describe and explain how TVIs and primary caregivers define the construct of kindergarten readiness for children who are blind and use braille as their primary learning medium. The primary research question guiding this study is: How is the construct of school readiness defined for children who are blind and use braille as their primary medium? Additional research questions include: (a)What is the current focus of instruction for children who are blind in preschool to prepare them for kindergarten? (b) What is the role of the TVI and primary caregiver in preparing students for kindergarten? (c) What do TVIs and primary caregivers identify as the barriers to school readiness for children who are blind? (d) What do TVIs and primary caregivers see as essential to preparing children who are blind for kindergarten? 
Qualitative methods were used to gather data from TVIs and primary caregivers of children who are blind in Oregon. Participants in the study were selected from TVIs currently teaching in Oregon who were teaching students with vision impairments and using braille during ECSE services. I used a combination of interview and document review. Primary caregivers were selected on a voluntary basis through a referral from the TVI providing education services for their child. The qualitative method was selected to allowed me to use open-ended questions, obtain ideas and in depth verbatim statements. Participants were recruited through the Oregon TVI listserv.

In addition to interview data, an examination of documents was used to gather data regarding school readiness skills for preschool students who are blind and use braille as their primary medium. Data was gathered from IFSP and IEP documents identified goals and objectives prioritized by the TVI during the two years of preschool prior to entering public kindergarten and during the kindergarten year. Documents were analyzed for trends and alignment with the Head Start Early Learning Outcomes Framework (USDHHS, 2015) and analyzed for trends and alignment with the Expanded Core Curriculum (Hatlen, 1996).

\section{Definition of Key Constructs}

The following are key terms defined for the reader to assist in in understanding the key constructs used in this research.

\section{Individuals with Disabilities Education Improvement Act}

The Individuals with Disabilities Education Improvement Act (IDEIA) is federal law that governs how public school districts and state agencies provide special education to students with disabilities (USDOE, 2004). IDEIA is a reauthorization of original 
legislation entitled Education for All Handicapped Children Act, passed in 1975. IDEIA mandates all public schools districts receiving federal dollars to be required to provide students with disabilities "a free and appropriate public education that emphasizes special education and related services designed to meet their unique needs and prepare them for further education, employment, and independent living" (USDOE, 2004).

Education for All Handicapped Children was amended in 1986 and renamed in 1990 as the Individuals with Disabilities Education Act, reauthorized in 1997 and then again in 2004 (Skiba, Simmons, Ritter, Gibb, Rausch, ... \& Chung, 2008; Yell, Rogers, \& Rogers, 1998). In defining the purpose of special education, IDEIA 2004 clarifies Congress' intended outcome for each child with a disability:

Students must be provided a free appropriate public education (FAPE) that prepares them for further education, employment and independent living. Special education and related services should be designed to meet the unique learning needs of eligible children with disabilities, preschool through age 21 and students with disabilities should be prepared for further education, employment and independent living (USDOE, 2004).

This research works within the framework of IDEIA and focused on students who were eligible for special education based on IDEIA.

\section{Disability}

A legal designation under IDEIA defines a child with a disability as having one or more of the following eligibilities: intellectual disability, hearing impairment (including deafness), speech or language impairments, visual impairments (including blindness), emotional disturbance, orthopedic impairments, autism, traumatic brain injury, other 
SCHOOL READINESS: CHILDREN WHO ARE BLIND

health impairments, or specific learning disabilities. Eligibility for special education requires identification in one of the disabilities areas AND the need for specially designed instruction and related services (USDOE, 2004). For the purposes of this paper, disability was defined as meeting the requirements of IDEIA and Oregon.

\section{Early childhood special education (ECSE)}

In 1986, after years of evidence documenting the effectiveness of early intervention as well as advocacy efforts by disability rights groups, Congress passed an amendment to P.L. 94-142 (The Education of the Handicapped Act), which required states to begin providing expanded services to children age birth to five by the school year 1991-1992 (Peterson, 1987; Yell, Rogers, \& Rogers, 1998). This amendment, Public Law 99-457 has two parts: Part B outlines services for children ages three through age five, and Part $\mathrm{C}$ outlines children from birth through age three. For children and their family, services include components such as an assigned case manager, the development of an IFSP the development of a multidisciplinary, coordinated interagency model of service delivery, and procedural safeguards (Peterson, 1987). Additional components include identifying the family as the central focus of services and providing for a smooth transition as the family moves from one services/system to another (Odom, \& McLean, 1996; Peterson, 1987). In this paper, ECSE referred to children ages three to five who have identified disabilities who may or may not have been enrolled in formal preschool settings but have received services in accordance to Public Law 99-457.

\section{Individual Family Service Plan/Individual Education Plan}

An Individual Family Service Plan (IFSP) is a family centered plan developed by a multi-disciplinary team to identify specially designed instruction and supports to assist 
a child in gaining skills to address identified developmental delay (USDOE, 2004). IFSP teams meet initially to establish eligibility as a child with a disability under the identified categories of IDEIA (USDOE, 2004). The plan must include an assessment of the child's present level of development, identified goals, supplementary services that will support the child to achieve those goals and frequency of the intervention provided, by who and where the services will occur (McGonigel, 1991). IFSP teams meet every six months to update the information and develop new goals when needed (USDOE, 2004). In most states, an IFSP is developed only for children age birth through age three at which time the student moves to an Individualized Education Plan (IEP). In Oregon, children have an IFSP until they transition to school age services.

An Individualized Education Plan is a plan developed by the educational team including the primary caregiver to identify educational instruction including accommodations and modifications provided for a child with a disability. The IEP process includes a determination of the least restrictive educational placement (USDOE, 2004). In Oregon an IEP developed as a child transitions into kindergarten and begins to receive services in K-12 public school.

\section{Child who is blind}

For the purposes of this paper, a child who is blind meets the legal definition of legal blindness which is an acuity of 20/200 or less in the better eye with best correction or a field loss such that there is less than 20 degrees of vision, or a progressive eye disease that leads to either of these conditions in the future. In addition to legal blindness, the IFSP or IEP team has determined through a learning media assessment that the child is a tactile learner and braille is the primary learning medium. 


\section{School readiness}

In this research school readiness was defined as a child who is blind and using braille has skills in the areas of child development and early learning as outlined by the Head Start Early Learning Outcomes Framework (USDHHS, 2015). The framework was intended to outline essential areas of development and learning to "establish school readiness goals for children, monitor children's progress, align curricula, and conduct program planning" (USDHHS, 2015, p. 2). When a child is ready for school, they demonstrate skills and development in the following domains: Approaches to learning, social and emotional development, language and communication, literacy, cognition including mathematics development and scientific reasoning, and perceptual, motor, and physical development. Skill development and progress should be reflected in the present levels of development, goals and objectives identified by the IFSP team between the child's ages three to five.

School readiness was also examined through the framework of the Expanded Core Curriculum (ECC) (Hatlen, 1996). The ECC does not have skills identified by ages but does guide instructional focus for TVIs in the following areas: Compensatory or Functional Academic Skills, including Communication Modes, Orientation and Mobility, Social Interaction Skills, Independent Living Skills, Recreation and Leisure Skills, Career Education, use of Assistive Technology, and Sensory Efficiency Skills.

\section{Oregon kindergarten readiness assessment}

With an acknowledgement that all children enter kindergarten at different stages in learning and development, the Oregon Early Learning Council along with the ODE developed and implemented The Kindergarten Readiness Assessment (KRA). The KRA 
is administered to every kindergarten student who attends a public school in Oregon before they begin their kindergarten year. The assessment is administered one to one with the certified kindergarten teacher. The assessment takes up to 20 minutes and assesses knowledge in social-emotional development, self-regulation, and early literacy and math skills. The intent of the assessment is to get a clearer picture of early learning experiences across the state, to aid teachers in better understanding their students' skills and needs and to begin to address the equity gap among underserved and disadvantaged early learners (Oregon House Bill 4165 Joint Workgroup Report, 2012). 
SCHOOL READINESS: CHILDREN WHO ARE BLIND

\section{Chapter 2: Literature Review}

In the previous chapter, I discussed the background and unique educational implications for a child and their teacher when a child is blind and uses braille as their primary learning medium. Children who are blind and entering kindergarten have additional and more complex needs than their sighted peers. The purpose of this research was to describe and explain how Teachers of the Visually Impaired (TVI) and primary caregivers define the construct of kindergarten readiness for children who are blind, identified as tactile learners, and use braille as their primary learning medium

In this chapter, I review the literature related to school readiness for students who are typically developing, children with disabilities and research related to school readiness for students who are blind. This chapter focuses on the following areas: (a) theoretical framework of the research; (b) literature regarding students without disabilities and school readiness including nonacademic and academic components; (c) literature regarding students with disabilities and school readiness, including nonacademic and academic components; and (d) literature regarding students with disabilities, specifically students who are blind and school readiness, including nonacademic and academic components. At the conclusion of this chapter, I present a rationale for research and methodology for my study.

\section{Theoretical Frame}

In the following section I present the relevant theoretical framework for this research, as well as present a rationale, implications and critique of the framework.

\section{Ecological and Dynamic Model of Transition}


This research is grounded in Rimm-Kaufman and Pianta's (2000) Ecological and Dynamic Model of Transition. Within this theoretical framework, family, primary caregivers, and teachers are critical in both preschool and kindergarten and play key roles in the transition between the two systems. Neighbors and peers also play critical roles in the model, however, for the purposes of this study, teachers and primary caregivers will be the focus of research.

Rimm-Kaufman and Pianta (2000) cited as influences of their theoretical model the increasing demands of public education as a result of national educational goals including school readiness in the transition to kindergarten. Rimm-Kaufman \& Pianta (2000) stated, "The primary advantage of research based on the Ecological and Dynamic Model of Transition is that it presents a more comprehensive explanation of the factors that contribute to children's transition" (p. 505). The framework focuses on the interrelationships of child, home, and school. Peer and neighborhood factors create a network of relationships that influence a child's transition to kindergarten both directly and indirectly (Rimm-Kaufman \& Pianta, 2000). I propose this network of relationships influences both directly and indirectly how school readiness is defined for a child given their unique context. Rimm-Kaufman and Pianta (2000) stated:

This model posits that the transition to school takes place in an environment defined by many changing interactions among child, school, classroom, family, and community factors. Child characteristics and contexts interact through a transactional process. These interactions, over time, form patterns and relationships that can be described not only as influences on children's development, but also outcomes of their own right. (p. 499) 
This research will focus on child characteristics of school readiness in the context of preschool and the context of the kindergarten classroom from the perspective of family and teachers of the visually impaired.

\section{The Ecological and Dynamic Model of Transition}

Preschool

Kindergarten

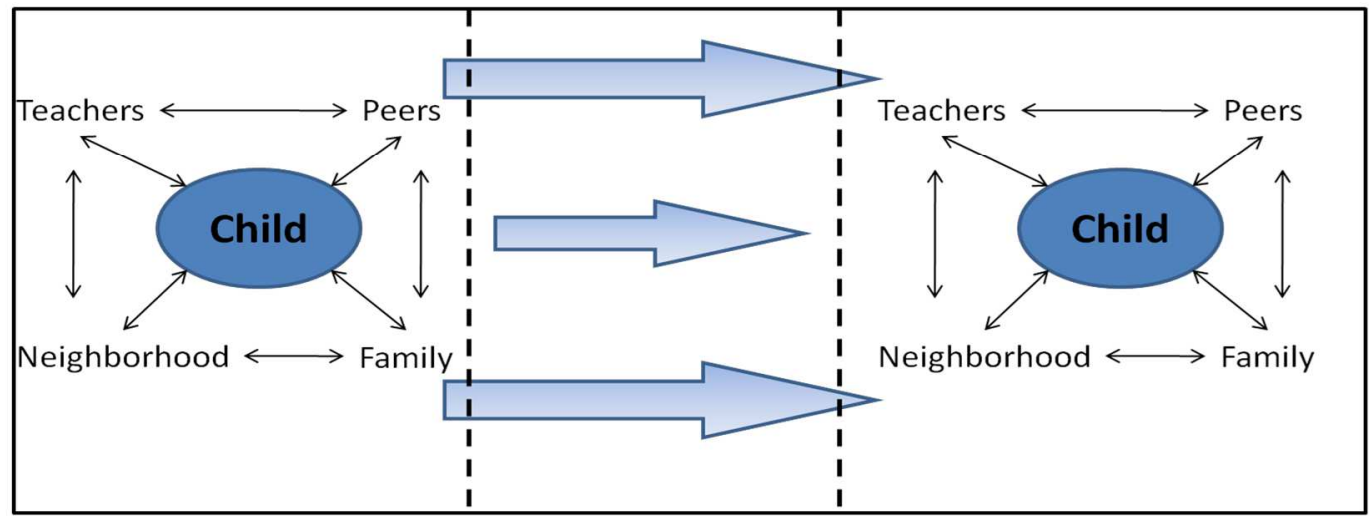

Figure 2.1 The Ecological and Dynamic Model of Transition. (Rimm-Kaufman \& Pianta, 2000, p. 497).

\section{Implications of The Ecological and Dynamic Model of Transition}

Key components of the Ecological and Dynamic Model of Transition (RimmKaufman \& Pianta, 2000) are grounded in critical components of the transition from preschool to kindergarten, which are critical to children who are blind. Rimm-Kaufman and Pianta (2000) noted the period roughly from age four to seven is identified as a period of change in the "developmental agenda" (p. 43). This period culturally in the United States marks a time when children are expected to begin to increase their independence and responsibility, their social networks begin to change from primarily adult-directed to peer-directed (Rimm-Kaufman \& Pianta, 2000; Edwards \& Whiting 1988). In addition, there is evidence of shifts in cognitive development including 
enhanced memory, new reasoning abilities, new strategies for recall in addition to physiological changes during this developmental time (Flavell, 1988; Nelson, 1996; Rimm-Kaufman \& Pianta, 2000; Stauder, Molenaar, \& van der Molen, 1993; Thatcher, 1994). The "developmental agenda" (Rimm-Kaufman\& Pianta, 2000, p.43), is based on research of sighted children. There are unique developmental implications for a child who is blind including reluctance from teachers and caregivers to allow for physical independence (Stuart, Lieberman, \& Hand, 2006), implications for peer relationships (Pérez-Pereira \& Conti-Ramsden, 2013) and unique cognitive implications (Fraiberg, 1977; Lowenfeld, 1981, Warren, 1984) based on a lack of foundations concepts due to vision loss (Erickson \& Hatton, 2007; Hatlen, 1996; Landau, Gleitman, \& Landau, 2009;; Perez-Pereira \& Conti-Ramsden, 2013; Preisler, 1995; Urwin, 1984a, Urwin, 1984b; Wormsley \& D'Andrea, 1997).

In addition to the internal changes within the child, the environment of kindergarten is different than either home or preschool. Goals, demands, and the nature of the classroom environment are different, as is the ecology surrounding this new environment (Rimm-Kaufman \& Pianta, 2000). Kindergarten typically has explicit goals for literacy, numeracy, and socialization not formally stated goals in preschool or home environments (Haines, Fowler, Schwartz, Kottwitz, \& Rosenhoetter, 1989; RimmKaufman \& Pianta, 2000). The concept of formal instruction with academic and social emotional expectations begins in earnest when a child enters formal schooling. Changing expectations in turn change the relationships between teachers and children, school and families. Contact with families becomes less frequent, more formalized and school directed. There is an increase in student-teacher ratio and changing expectations between 
teacher-child interactions including an increase demand for attention to teacher directed tasks. Rimm-Kaufman and Pianta (2000) noted the implications for social and emotional demands:

The new demands of kindergarten place stress on social and emotional competencies as well. Demands such as independence from adults, getting along with other children, recognition and adherence to routine, and being alert and active for longer periods of time can challenge the 5-year-old child. (p. 494) The new demands of kindergarten impact sighted children as well as children who are blind.

In kindergarten teacher expectations of children in kindergarten become more focused on academic skills, activities are more structured and teacher-directed with less unstructured student-directed time. Children spend more time in large groups with less individualized instruction, and "the emphasis on academic skills and the demands to interact with a wide range of children are reported to be the most difficult aspects of the transition to school" (Rimm-Kaufman \& Pianta, 2000, p. 494). There is also the complexity of a TVIs role as part of the transition. TVIs are part of a blind child's unique ecological context. They are highly specialized teachers who have expertise in the disability of vision impairment and blindness. There is often a close, unique relationship between the TVI, the child and family that may influence the teacher's assessment of the student's skills and abilities. In my experience, often the TVI is the consistent teacher from preschool to formal schooling.

Finally, Rimm-Kaufman and Pianta (2000) emphasized the importance of the first formal educational experience for children and their later academic success. Researchers 
suggested the first years of a child's education achievement and outcomes are predictors of later academic and school success in later grades (Bailey, 2014; Duncan et al., 2007; Entwisle \& Alexander, 1996; Farran, 2011; Goldstein et al., 2013; Kagan \& Rigby, 2003; Kern \& Friedman, 2009; Konold \& Pianta, 2005; Lloyd, Irwin, \& Hertzman, 2009; Magnuson et al., 2007; Pianta \& Walsh, 1996; Ramey \& Ramey, 2004; Rimm-Kaufman \& Pianta, 2000). Given children who are blind often begin their education behind their sighted peers, it is crucial that the time of transition and school readiness is a focus for TVI and primary caregivers. The work in preschool and transitions lays the groundwork for future success.

\section{Critique of the Ecological and Dynamic Model of Transition}

The Ecological and Dynamic Model of Transition (Rimm-Kaufman \& Pianta, 2000) captures the importance and breadth of this time in a child's life. In addition, Rimm-Kaufman and Pianta (2000) suggested this theoretical framework is best suited and substantiated by research focused on transitions for students with identified disabilities (Fowler, Schwartz, \& Atwater, 1991; Katims \& Pierce, 1995; Repetto \& Correa, 1996; Roberts, Akers, \& Behl, 1996; Rous, Hemmeter, \& Schuster, 1994). There is a focus on changing contexts and relationships over time for children with identified disabilities, transitioning from home to preschool and preschool to formal schooling. The focus of this theoretical construct is not solely a child-centered perspective identifying child competency but also includes the influence of multiple contexts, including home, school, peers and neighborhood. For a child who is blind, these contexts have a significant influence on access to academic, social and behavioral competencies. The 
SCHOOL READINESS: CHILDREN WHO ARE BLIND

final components critical for a child who is blind are the relationships among contexts including teachers, specialists, families and peers.

A critique of this theoretical framework would be the limited research and longitudinal studies of the framework over time. In addition, this framework is not specifically grounded in transition for students who are blind or visually impaired. An additional critique of this frame is the lack of specific guidance on development of school readiness skills. This framework can be seen as more suited to social emotional and relationship considerations rather than academic readiness and curriculum.

A final critique of this framework is the difficulty in isolating specific variables. It would be difficult to use this model consistently across students, as there is a continual change and influence across many contexts. This makes it difficult to recommend specific interventions that would consistently support a variety of children in the many contexts in which they experience the transition from preschool to kindergarten.

\section{Review of the Literature}

I began the review of literature by framing for the reader the developmental profile of children who are blind and provided information on the two most consistently used developmental assessments for preschool children who are blind. I then examined the literature regarding the construct of school readiness for typically developing children, children with disabilities and specifically children who are blind. I considered the historical perspective of children identified as "ready" for school. Then, I examined the literature for children who are sighted and for children who are blind. I explored this literature from the perspective of kindergarten and preschool teachers, teachers of the visually impaired, and primary caregivers of children who are blind. Using the 
Ecological and Dynamic Model of Transition (Rimm-Kaufman \& Pianta, 2000), these perspectives were interwoven and considered in relation to one another and wholly with the child squarely centered in the research.

\section{Developmental Profile of Children who are Blind}

Developmental milestones and profiles for children who are blind are unique to each individual child and dependent on the etiology of their vision loss, severity and age of impact and co-occurring disabilities (Hatton, Bailey, Burchinaland, \& Ferrell, 1997). For children with severe vision loss resulting in the use of braille, researchers have identified delays in global development when compared to sighted children (Ferrell, 1986; Fraiberg, 1977; Hatton et al., 1997; Reynell, 1978). Those areas include cognitive development, social and emotional development, language development, and gross and fine motor development (Hoben \& Lindstrom, 1980, MacCuspie, 1996; Warren, 1984). Some researchers suggest delays may be due in part to deficiencies in stimulation to all the senses or the lack of motivation, overprotection, or the fear of actual or perceived dangers (Rettig, 1994; Schneekloth, 1989). Research in these areas is summarized below.

Cognition. Researchers have suggested there is a possibility that cognitive abilities develop more slowly or in a different way for children who are blind (Fraiberg, 1977; Lowenfeld, 1964; Warren, 1984). Lowenfeld (1948) suggested lack of vision impacts cognitive development by restricting the range and variety of experiences, the ability to move in and around the environment, and control of the environment and self in relation to the environment. Foulke, Amster, Nolan, and Bixler (1962) noted the lack of vision creates restricted experiences for the child who is blind and that touch does not serve to mediate two-dimensional representations of three-dimensional objects 
(Warren, 1984). Children with significant vision loss do not reach for objects or move out into their environment until they understand objects exits. Hatton, Bailey, Burchinaland, and Ferrell (1997) have suggested cognitive and motor development is "inextricably linked in early development of children who are visually impaired" (p. 802). In addition, the child who is blind is more dependent on secondhand experience conveyed verbally from other people therefore they are more dependent on verbal development and facility to reach and achieve cognitive milestones (Perez-Pereira \& Conti-Ramsden, 2013; Warren, 1984).

Early researchers focused attention on sighted children understanding properties of the world including object permanence, classification and conservation (Piaget, 1952). For children who are blind, researchers have noted significant developmental delays in reaching these milestones (Fraiberg, 1977; Warren, 1984). This is not to say there are intellectual delays, but delays in reaching these milestones "because he cannot obtain the prerequisite data from his surroundings" (Higgins, 1973, p. 37). Of note is that touch for children who are blind does not serve the same function as sight does for the sighted child (Warren, 1984). Children who are blind are often delayed in reaching, crawling, and object exploration (Fraiberg, 1977; Landau, 1991; Norris, Spaulding, \& Brodie, 1957). Warren (1984) further notes that children who are blind often have developmental lags in several areas of development including motor, language, and cognitive skills.

Social and emotional development. When children are blind, they are dependent on familiar voices and experiences in interaction to understand themselves in relation to others which results in limited early social experiences and leads to long term difficulties in social understanding (Brown, Hobson, Lee, \& Stevenson, 1997). 
The emotional development of children who are blind may be at risk due to the constraints on the child's capacity to share and respond to the feelings of others (McAlpine \& Moore, 1995).

A review of literature suggests children who are blind have deficits in play and these deficits are related to delays in several social and emotional domains (D'Allura, 2002; Erwin, 1994; Fraiberg, 1977; Fraiberg, Smith, Adelson, 1969; Rogers \& Puchalski, 1984). Significant areas of delay are the areas of play and social interaction as well as development of self and self-awareness (Rettig, 1994). Schneekloth (1989) and Erwin (1993) observed children who are blind spend more time playing alone and more time in adult interactions versus peer interaction. Children who are blind tend to be egocentric and more interested in their own bodies than in their environment (Parsons, 1986). Warren (1984) suggested that self-centeredness or egocentrism observed in children who are blind is a result of limited social understanding. Children who are blind are much more likely to be the recipients, rather than initiators of interaction (D'Allura, 2002).

Early social emotional developmental milestones may be delayed for children who are blind including the development of theory of mind, the idea that people can make sense of others' behavior by hypothesizing about feelings, desires, and beliefs that motivate their actions (Hughes \& Leekam, 2004). Understanding the intentions, feelings, and actions of others and reacting accordingly helps people navigate social interactions (Dunn, 1988; Hughes \& Leekam, 2004). Sighted children begin to develop this understanding typically around age four (Hughes \& Leekam, 2004). Theory of mind has a foundation in early childhood development in perspective taking and joint attention, two developmental milestones that are significantly impacted by lack of vision (Hughes 
\& Leekam, 2004). Joint visual attention allows an infant to participate in shared reference and communication about a shared experience. By the end of the first year, the use of eye gaze with pointing to clarify an object of shared reference and experience is recognized as an important cognitive milestone (Bates, 1979; Hughes \& Leekam, 2004; Piaget, 1955). Shared reference is further delayed and restricted to topics that are often confined to the environment in close proximity of the child and are mostly child centered (Kekelis \& Anderson, 1984). Children who are blind may not develop the ability to recognize vocally expressed emotions to compensate for lack of access to visual cues such as facial expressions, gestures, or body postures, leading to delays in the development of social understanding and social relationships with peers and adults (Hughes \& Leekam, 2004; Minter, Hobson, \& Pring, 1991).

With regard to imaginative play and use of toys, children who are blind spend a significantly lower percentage of time engaged in functional toy use and engaged in significantly more stereotypical behavior during play (Parsons, 1986; Rettig, 1994). Children who are blind generally display less creativity and imagination in their play and are less interested in toys and play than their sighted peers (Rettig, 1994; Warren, 1984). Children who are blind do not generally reach out for toys and need extra time and support to discover what and where their toys are and what to do with them (Rettig, 1994).

Language development. Language has many functions including communication, social interaction categorization and organization of thought (Pring, 2005). Language development may be impacted immediately at birth if a child has no vision to establish eye contact with primary caregivers, a critical factor in attachment and 
socialization process (Fraiberg, 1977; Preisler, 1995; Rogers \& Puchalski, 1984; Troster \& Brambring, 1992). "Preverbal communication, particularly imitation and social reciprocity, is highly dependent on vision, as infants and parents learn to read and respond to each other, both socially and verbally, through visual observation and attention" (Hatton et al., 1997, p. 788). Researchers have suggested that although children with visual impairments acquire language within the same age range as sighted children, there are documented differences in the types of words acquired and in the use of words (Andersen, Dunlea, \& Kekelis, 1984; McConachie \& Moore, 1994). Researches have identified challenges with reciprocity, pragmatics and referentialism (Andersen et al., 1984; Bigelow, 1987; Dunlea, 1989; Hatton et al., 1997; McConachie \& Moore, 1994; Mills, 1988; Mulford, 1988; Preisler, 1995). Finally, researchers have reported delays in attainment of object concept by children with significant vision impairments (Bigelow, 1990; Fraiberg, 1977; Hatton et al., 1997; Rogers \& Puchalski, 1988).

There is a noted relationship between play and language development, particularly the relationship of symbolic play and the use of "I" and "no" (Rettig, 1994). Children who display symbolic play are more likely to use the word "no" and to use two word sentences (Rodgers \& Puchalski, 1984). Rodgers and Puchalski (1984) also point out that the use of the word "no" is a critical step in a child's sense of autonomy. Children who are blind do not show signs of imaginative play until much later than their sighted peers, delaying the use of pronouns.

Children who are blind ask more questions of adults than their sighted peers (Tait, 1972). Researchers suggest they ask more questions to further their understanding of the 
environment, to gain information or to gain assurance before attempting an action (Fraiberg \& Adelson, 1973; Rettig, 1994; Rogers \& Puchalski, 1984). Children who are blind also use the strategy of asking questions to maintain open lines of communication with adults (Rettig, 1994).

Gross and fine motor development. Delays in reaching motor milestones and qualitative differences in locomotion and fine motor skills have been documented by many researchers (Brown \& Bour, 1986; Ferrell, Trief, Deitz, \& Bonner, 1990; Fraiberg, 1977; Hatton et al., 1997; Norris et al., 1957; Troster \& Brambring, 1993). Children who are blind are typically less active than their sighted peers which some researchers attribute to fear of movement, spatial disorganization, hypotonic, and parental fear of harm and subsequent restrictions (Brown \& Bour, 1986; Hatton et al., 1997; Jan, Robinson, Scott, \& Kinnis, 1975; Sonksen, Levitt, \& Kitzinger, 1984). Delayed development in object concept has also been associated with delays in reaching and locomotion (Fraiberg, 1997; Hatton et al., 1997; Troster \& Brambring, 1993). In totality, these factors interact to delay motor development and also lead to restricted direct experiences with the environment that facilitate cognitive and language development (Hatton et al., 1997).

Assessments. To assess developmental and skill progression the most consistently used developmental assessment in Oregon for preschool children who are blind is The Oregon Project for Blind and Visually Impaired Preschoolers - Skills Inventory (Brown et al., 1978). Additional developmental assessments often used are the Assessment, Evaluation, and Programming System for Infants and Children (Bricker \& 
Squires, 1999) and the Ages and Stages Questionnaire (Squires, Bricker, \& Twombly, 2002).

The Oregon Project for Blind and Visually Impaired Preschoolers - Skills Inventory (The OR Project). The OR Project (Brown et al., 1978) is criterionreferenced and measures performance of individual skills based on observations of teachers and caregivers. The inventory is specifically for children from birth to age six and supports teachers, parents or staff to determine a performance level of a visually impaired or blind child. The inventory includes corresponding curriculum and activities to support development of specific skills. The skills inventory consists of over 800 behavioral statements organized in eight developmental areas: Cognitive, Language, Socialization, Vision, Compensatory, Self Help, Fine Motor, and Gross Motor. Each of the eight areas contains skills which have been developmentally sequenced and arranged in age categories: birth-1, 1-2, 2- 3, 3-4, 4-5, and 5-6 years. Skills are identified as needed by a visually impaired or blind child in preparation for kindergarten. The OR Project (Brown et al., 1978) is taught in the Portland State University Visually Impaired Learner program and is consistently implemented in Oregon by TVIs.

Assessment, Evaluation, and Programming System for Infants and Children (AEPS). The AEPS (Bricker \& Squires, 1999) is a criterion-referenced assessment often administered to children receiving services from early childhood special education providers in Oregon. According to the authors, the AEPS is designed to be used by qualified staff including early childhood special educators Bricker \& Squirs, 1999).

Ages and Stages Questionnaire (ASQ). The ASQ (Squires et al., 2002) is a questionnaire designed to be completed by the primary caregiver. The ASQ 
(Squires et al., 2002) is a first-level screening tool designed as an early identification means for children at risk for social or emotional difficulties. It is not a diagnostic tool but a tool to identify if children need further evaluation or specific interventions in these areas. The ASQ (Squires et al., 2002) has a series of eight questionnaires that correspond to age intervals from birth to age six. Parents or primary caregivers answer questions that are then scored to determine if the child's development appears to be progressing as expected.

\section{Historical Perspective of School Readiness}

When examining the concept of school readiness, it is necessary to look at the concept through a historical perspective. According to Kagan and Rigby (2003) the concepts of readiness have been noted in research as far back as 1898 when Pestalozzi investigated the concept as well as the work of Gagne and Piaget in examining their respective concepts of readiness and learning. Attention was also given to the importance of readiness by the International Kindergarten Union as early as 1920 (May \& Campbell, 1981). At that meeting a reading readiness committee was formed to examine a better understanding of the concept of readiness and its role in the reading processes. The current-day concept of school readiness has its' roots in the establishment of National Education Goals Panel (NEGP). In 1990, President George H. Bush, along with the nation's Governors, created the National Education Goals with a target date of achievement by the year 2000. The panel and goals were a result of A Nation at Risk (Gardner, 1983) a report of the then-failing school system in the United States. The first goal states "by the year 2000, all children in America will start school ready to learn" (National Education Goals Panel, 1995, p. XIV). Readiness in children was and still is a 
term that is defined broadly from many perspectives (Dockett \& Perry, 2002; Farran, 2011; Gill, Winters, \& Friedman, 2006; Kagan, 1990; Kagan \& Rigby, 2003; Konold \& Pianta, 2005; May \& Campbell, 1981; Raver \& Knitzer, 2002). The NEGP went beyond academically driven definitions of readiness to a broader definition that included physical, social, and emotional as well as cognitive readiness. Researchers still agree the term school readiness is multi-faceted and evolving based on cultural context, child specific factors and federal and state regulations regarding standards based education and academic outcomes (Farran, 2011; Kagan et al., 1995; Kagan \& Rigby, 2003; Konold \& Pianta, 2005; Raver \& Knitzer, 2002). The panel identified five components included physical well being and motor development, social and emotional development, approaches to learning, language development (including listening, speaking and vocabulary, emerging literacy, print awareness, story sense and phonemic awareness), cognition and general knowledge (including letter sounds, shapes, spatial relations and number concepts) (Kagan et al., 1995).

There is no question that school readiness is a priority in our nation and in Oregon (Hammond, 2012; Johnson, 2015, Kagan \& Rigby, 2003). Goal One of NEGP is for all children to enter school ready to learn. Goal one of the Oregon Investment Education Board (OIEB) is "more children ready for school," defined as children entering kindergarten knowing letters and sounds and how to count (Oregon House Bill 4165 Joint Workgroup Report, 2012). The measure of a child's school readiness in Oregon is the Kindergarten Readiness Assessment (KRA), a measure of cognitive, social, emotional and behavior skills.

The difficulty with the concept of school readiness is a lack of agreement of the 
definition and scope of readiness skills (Dockett \& Perry, 2002; Kagan \& Rigby, 2003). In addition, the concept of readiness in relation to students with disabilities has a history of being a concept used to keep children out of typical classrooms (Kagan \& Rigby, 2003). The concept of readiness at one point was seen as a set of skills a student needed before they were "ready" for the classroom; if the student did not demonstrate these skills, they were kept out of school. Now, however, states have revised their kindergarten entry based on a student's chronological age rather than a set of readiness skills. Yet, even with this revision, the concept of readiness has implications for a student's experience in the transition to kindergarten as well as their future academic experiences and success.

Goal One of NEGP began a national dialogue of the concept of readiness (Kagan, 1990; Scott-Little et al., 2006). "The challenge of conceptualizing and articulating the notion of readiness is not new and is a complex and multi-faceted issue" (Scott-Little et al., 2006, p. 154). There are historical references to readiness (May \& Campbell, 1981; Pestalozzi \& Cooke, 1898; Scott-Little et al., 2006), however the concept of readiness for learning and readiness for school have seen specific focus since the inception of the National Goals (Kagan, 1990). The concept of "readiness for learning" has generally been conceptualized as a developmental progression that includes a maturational view of readiness for school (Kagan, 1990). "Readiness for school" has generally been viewed as a fixed or prerequisite set of physical, intellectual and/or social skills needed for children to be able to fulfill the requirements of the school environment (Kagan, 1990). "The conceptualization of school readiness as something that exists within the child focuses on readiness as a determined set of skills and knowledge that are prerequisites for 
later success in school" (Scott-Little et al., 2006, p. 155). This necessitates the need to define what the specific set of skills and knowledge are that children need as they enter kindergarten.

Researchers suggest if children enter kindergarten at a disadvantage, lack of skills and understanding academic and social emotional concepts and expectations tend to be sustained and even widen over time (Aunola, Leskinen, Lerkkanen, \& Nurmi, 2004; Linder, Ramey, Zambak, 2013). Research on teacher and parent belief of readiness skills suggests that adults' views of readiness vary based on social and cultural contexts (Graue, 1993; Meisels, 1999; Scott-Little et al., 2006; Smith \& Shepard, 1988). There is agreement among teachers (both preschool and kindergarten) of the importance for children to be healthy, socially competent, able to communicate effectively and able to follow a teacher's directions as skills necessary to be ready for kindergarten (Linder et al., 2013; Scott-Little et al., 2006; Wesley \& Buysse, 2003). While social emotional and physical aspects of readiness are widely accepted, there are differences in the priority and importance of academically related skills (Haines et al., 1989; Harradine \& Clifford, 1996; Scott-Little et al., 2006; West, Jausken, \& Collins, 1993). The work of the NEGP conceptualized a broader definition of readiness to include a multi-faceted construct that includes families, preschool and early education programs, culture and community as well as the child's unique characteristics and the capacity of the school to effectively educate the child once they begin kindergarten (Graue, 1993; Hyson \& Biggar, 2006; Kagan, 1990; Kagan et al., 1995; Kagan \& Rigby, 2003; Scott-Little et al., 2006; ScottLittle \& Maxwell, 2000). This construct of readiness is in alignment with the Ecological and Dynamic Model of Transition (Rimm-Kaufman \& Pianta, 2000) and the unique 
needs of children who are blind.

In discussions of school readiness Scott-Little, Kagan and Frelow (2006) focused on an examination of early learning standards because, "early learning standards that define expectations for children's learning and development prior to kindergarten entry are, in essence, a reflection of how states are conceptualizing children's readiness for school" (p. 153). In their study, they found since 1999, 46 states have adopted early learning standards for children age three to five. In the Oregon, the Head Start Early Learning Outcomes Framework (USDHHS, 2015) was adopted as early learning standards for children age three to five, summarized in Table 1.1.

Scott-Little et al. (2006) found that of all possible domains, consistent focus of early learner standards and consequently school readiness were cognition and general knowledge, followed by language and communication development. According to ScottLittle et al. (2006), cognition and general knowledge included mathematical knowledge, social and social-conventional knowledge and knowledge of the physical world.

Language and communication development included verbal language (social and creative uses of language, speaking, creative expression (non-verbal) listening, questioning and non-verbal communication as well as early literacy skills (writing, print awareness, vocabulary and meaning, phonemic and phonological awareness, alphabet and literature awareness, comprehension, book awareness and story sense) (Scott-Little et al., 2006). Based on the results of their study, Scott-Little et al. (2006) identified "It appears that children's readiness for school is being defined as specific sets of skills and knowledge that contribute to children's later success in school" (p. 163). In their study, $95 \%$ to $100 \%$ of preschool and kindergarten teachers identified academic skills such as counting to 10, 
naming colors and shapes, recognizing letters of the alphabet, and prewriting activities such as tracing and drawing as skills that promote school readiness (Scott-Little et al., 2006). In the area of social-emotional and behavioral, skills such as following directions, cooperation and working independently, play cooperatively, recognizing feelings and appreciation of their own and others cultures were also equally important and emphasized in both programs. This research is focused on identifying what the specific sets of skills and knowledge that TVIs and primary caregivers identify that contribute to later success in school for a child who is blind and uses braille as their primary learning medium.

\section{Literature based on children who are sighted}

There is extensive research covering the construct of school readiness skills for children who are sighted.

Table 2.1

Summary of literature search

\begin{tabular}{cccc}
\hline Search Engine & $\begin{array}{c}\text { Keywords: School } \\
\text { Readiness/Kindergarten } \\
\text { Readiness }\end{array}$ & $\begin{array}{c}\text { Keywords: } \\
\text { Adding Disability }\end{array}$ & $\begin{array}{c}\text { Keywords: } \\
\text { Adding Blindness }\end{array}$ \\
\hline $\begin{array}{c}\text { Education } \\
\text { Research } \\
\text { Complete }\end{array}$ & $578 / 53$ & 40 & 5 \\
ERIC (EBSCO) & $340 / 32$ & 15 & 2 \\
$\begin{array}{c}\text { Gales Education } \\
\text { Reference }\end{array}$ & $230 / 60$ & 20 & 0 \\
$\begin{array}{c}\text { Professional } \\
\text { Development } \\
\text { Collection }\end{array}$ & $228 / 17$ & 11 & 3 \\
Research Starters \\
- Education
\end{tabular}


Given the extensive amount of research regarding school and kindergarten readiness for typically developing children, a criterion for inclusion and exclusion of literature was necessary for the purposes of this study. For this research I used four criteria of inclusion (a) articles published after 2011; (b) peer-reviewed articles; and (c) articles based on rigorous research methods (Linder et al., 2013). In addition, I did not use articles that were specific to a state, country outside of the United States, specific populations (other than children who are blind), socioeconomic status and gender. I also used articles reflective of the whole child following the theoretical framework of The Ecological and Dynamic Model of Transition (Rimm-Kaufman \& Pianta, 2000).

Linder, Ramey and Zambak (2013) completed a literature review focused on school readiness in the areas of literacy and mathematics including publications from 1995 to spring of 2011. This following is a summary. Linder et al. (2013) focused their literature review on school readiness in the areas of literacy and mathematics, however, in their research, several themes developed which encompasses the five components identified by the National Education Goals Panel. Their purpose was to provide, caregivers and teachers information regarding specific factors that contribute to a child's successful inclusion into kindergarten. Their guiding question was "What predictors of school readiness in mathematics and literacy have been identified by empirical research in education?" (Linder et al., 2013, p. 2). Their method included a systematic review of the literature conducted over three months in the year 2011. They used four criteria for articles to be included: (a) only publications after 1995; (b) articles published in peerreviewed journals; (c) based in empirical research; and (d) articles based on rigorous research methods (Linder et al., 2013). The research team used electronic searches based 
on the criteria above. In addition, snowball sampling included reviewing the references from theoretical articles in the areas of school readiness, achievement, and early childhood literacy and mathematics (Linder et al., 2013). Linder et al. (2013) identified seven themes based on their review as factors associated with school readiness: child care experience, family structure and parenting, home environment, learning-related skills, social behavior, mathematical and literacy-based tasks, health and socio-economic status. What follows is a summary of themes identified by Linder et al. (2013) as well as how these themes manifest or influence the preschool experience for a child who is blind.

Child-care experience. Researchers suggested participation in high quality child-care that implements high-quality curricula was correlated to increased performance on measures of school readiness in literacy and mathematics (Klein, Starkey, Clements, Sarama, \& Iyer, 2008; Linder et al., 2013; Ramey \& Ramey, 2004; Magnuson et al., 2005). Ramey and Ramey (2004) identified specific experiences critical for healthy development to support behavioral and academic readiness for school. Those experiences included "(a) encourage exploration, (b) mentor in basic skills, (c) celebrate developmental advances, (d) rehearse and extend new skills, (e) protect from inappropriate disapproval, teasing, and punishment, (f) communicate richly and responsively, and (g) guide and limit behavior" (Ramey \& Ramey, 2004, p. 474). In addition, Chien et al. (2010) noted children in settings with more free play showed smaller gains than their peers who received individual instruction in the areas of math and reading. This research suggests children who are blind should attend formalized preschool (child-care setting) with high-quality curricula in literacy (braille) and mathematics. They will also benefit from individualized, direct instruction in areas of 
academics.

In Oregon, formal school begins at kindergarten, there is no universal preschool offered through the state education agency. Children who are blind are eligible for EI/ECSE services based on their visual impairment. In Oregon, however, this eligibility does not provide for preschool unless the team determines a specialized preschool as an educational placement. If a child has only vision impairment, specialized preschool placements may be too restrictive and not seen as a natural environment. Attendance in preschool is dependent on families' socio-economic status, working situation, and family dynamics. There may be a barrier for children who are blind attending private child care settings due to misunderstandings and low expectations of children who are blind.

Children who are blind may be perceived to be better educated in specialized classrooms or schools to meet their unique needs.

Home environment. Linder et al. (2013) noted several studies that identified a relationship between school readiness and home environment (Baroody \& Diamond, 2012; Burgess, Hecht, \& Lonigan, 2002; Clarke \& Kurtz-Costes, 1997; Murphy, Hatton, Erickson, 2008). Burgess, Hecht, and Lonigan (2002) completed a longitudinal study of children's reading abilities and the literacy environment in the home. They found the literacy environment in the home was significantly related to a child's ability in oral language, word decoding, and phonological awareness (Burgess et al., 2002). The home literacy environment was defined in two ways, passive or active (Burgess et al., 2002). Passive activities were "parental activities that expose children to models of literacy usage (e.g., seeing a parent read a newspaper)" (Burgess et al., p. 413, 2002). Active strategies were "parental efforts that directly engage the child in activities designated to 
foster literacy or language development (e.g. rhyming games, shared readings)" (Burgess et al., 2002, p. 413). However, Brennan, Luze, and Peterson (2009) commented on the difficulty parents who are sighted have in seeing their own capability to create an active home literacy environment for children who are blind. "Parents of a child who is a braille reader may view learning braille as difficult and similar to learning a foreign language" (Brennan et al., 2009). In the passive literacy environment, children who are blind are at a disadvantage. Unlike children who are sighted, children who are blind cannot participate in or benefit from incidental learning activities without direct involvement from teachers or caregivers to facilitate their interactions with the environment (Brennan et al., 2009; Koenig \& Farrenkopf, 1997).

Researchers identified parental involvement and expectations as a predictor of school readiness and academic achievement (Burgess et al., 2002; Clarke \& KurtzCostes, 1997; Entwisle \& Alexander, 1996; Hill, 2001). This includes the quality of parent-child interactions, maternal warmth, parental patience and perceptions of a child's ability. All of these constructs are impacted when a sighted parent is raising a child who is blind (Brennan et al., 2009; Koenig \& Farrenkopf, 1997; Murphy et al., 2008).

Learning-related characteristics. McClelland, Acock, and Morrison (2006) identified in the research behaviors and child dispositions positively related to school readiness including engaging and perseverance in tasks, task completion, motivation, following directions, taking turns in group activities, and on-task behavior. Selfregulation and social competence were also predictive of math and reading achievement beginning in kindergarten (McClelland, Acock, \& Morrison, 2006; Ramani \& Siegler, 2008; Weiland \& Yoshikawa, 2013). For children who are blind, engaging and 
perseverance in tasks, task completion, motivation, following directions, taking turns in group activities, and on-task behavior are often impacted by blindness (Bedny \& Saxe, 2012). Educators may have low expectations for children who are blind following and participating in large group instructional activities (Cutsforth, 1951; Wilde, 2009). In addition, children who are blind are potentially paired with a para-educator who provides direct instruction and support for these skills, sometimes creating a learned helplessness (Conroy, 2007, Giangreco, 2010).

Social behavior. Research has increasingly focused on social emotional behavior and competencies as indicators of school readiness (Blair, 2002; Denham \& Brown, 2010; Raver \& Knitzer, 2002; Rimm-Kaufman et al., 2002). Raver and Knitzer (2002) reported, "Across a range of studies, the emotional, social, and behavioral competence of young children (such as higher levels of self-control and lower levels of acting out) predict their academic performance in first grade, over and above their cognitive skills and family backgrounds" (p. 3). Denham and Brown (2010) reported increased recognition of the importance of social emotional skills has been crucial for the well being and mental health of the child as well as later academic success. Researchers suggested issues of emotional and behavioral concerns are among educators' top needs for training and professional development (Huffman et al., 2001; Peth-Pierce, 2000). Finally, teachers' views of readiness to learn are positive when children demonstrate behaviors including " emotional expressiveness, enthusiasm, and ability to regulate emotions and behaviors" (Denham \& Brown, 2010, p. 58). Other research suggests kindergarten teachers see a child's social development, persistence on task and curiosity as more important than academic readiness (Blair, 2002; Chien et al., 2010; Denham, 
2006; Dockett \& Perry, 2002; Gill et al., 2006; Justice, Petscher, Schatschneider, \& Mashburn, 2011; Konold \& Pianta, 2005; Magnuson et al., 2005; Peth-Pierce, 2000; Rimm-Kaufman et al., 2002; Sabol \& Pianta, 2012a). Given the recent emphasis on social emotional skills, it is critical these skills are addressed in early childhood special education for children who are blind. As previously stated, social emotional development for a child who is blind is at risk, there are delays in social skills, play skills and understanding of themselves and others. Social emotional skills require explicit and direct instruction in the development of relationship with adults and peers.

Literacy and mathematical based tasks. The core of a child's academic achievement and success is often attributed to literacy (Xue \& Meisels, 2004). Literacy and mathematic based tasks related to school readiness include examining concepts of print, shared story book reading, playing board games, counting and number sense, engaging in block building literacy concepts (phonological awareness, decoding, awareness of print, and letter identification (Hanline, Milton, \& Phelps, 2010; Ramani \& Siegler, 2008; Wolfgang, Stannard, \& Jones, 2001). These combined subjects are the core academic readiness skills for all children.

For children who are blind, much of the focus of early intervention and school readiness has been focused on braille instruction, specifically the mechanics of reading the tactile code. This will be discussed in greater depth when literature in this area is reviewed.

Ecological and Dynamic Model of Transition and readiness. Since 2011, researchers have continued to focus on readiness skills for typical children entering kindergarten. A relatively recent construct of school readiness is the notion of a person 
centered approach (Sabol \& Pianta, 2012b), which attempts to capture a more holistic view of the child's performance as opposed to looking at sets of scores on assessment batteries. In addition, the notion of executive functioning skills has appeared in the literature as descriptive of preschool children's cognitive ability (Fitzpatrick, McKinnon, Blair, \& Willoughby, 2014; Fitzpatrick \& Pagani, 2012; Sabol \& Pianta, 2012a; Shaul \& Schwartz, 2014). In fact, when asked, kindergarten teachers identify areas related to executive functioning as being most critical to school readiness including concentration, persistence, paying attention to teacher-directed activities, following rules, focusing on activities independently, cooperation, positive peer and teacher interactions, appropriate emotional responses, self initiation of activities and handling transitions (Chien et al., 2010; Farran, 2011; Rimm-Kaufman et al., 2002; Sabol \& Pianta, 2012a; Shaul \& Schwartz, 2014). These skills are referred to in the literature as executive functioning skills, self-regulation, learning dispositions and approaches to learning (Farran, 2011). In Oregon, with the adoption of the Head Start Early Learning Outcomes Framework (USDHHS, 2015) approaches to learning have been identified as the State Early Childhood Outcome goal for the current year.

School readiness is a function of many variables including the child, their home and socioeconomic status as well as the early childhood experiences they bring to kindergarten. Researchers have begun to examine the nature and quality of early childhood experiences that children receive prior to kindergarten. Children who qualify for Early Childhood Special Education are required to receive services beginning at age three. The type and quality of those services is also the subject of research. A common theme of current research is school readiness as multidimensional. "Currently, there is an 
emphasis on both cognitive/pre-academic skills, communication skills, and socialemotional/learning behaviors" (Goldstein et al., 2013, p. 508). Oregon attempted to capture kindergarten readiness by using the Head Start Early Learning Outcomes Framework (USDHHS, 2015) to develop the Kindergarten Readiness Assessment.

\section{Readiness and Children who are Blind}

Expectations for behavior and academic performance are changing in kindergarten (Farran, 2011). Kindergarten in the United States now requires children to learn content reserved for grades one and two in the past (Goldstein, 2007; Litty \& Hatch, 2006). Both the content and the pedagogical approach have changed dramatically, becoming more rigorous, with more direct instruction and less child initiated learning" (Farran, 2011, p. 8). For children who are blind, it is critical that teachers of the visually impaired understand the changing nature of kindergarten and how to teach children who are blind the skills they need for the general education classroom. As identified in research, it is important to focus on school readiness that includes cognitive/pre-academic knowledge and skills, communication skills including both receptive and expressive language, and the development of social-learning behavior (Farran, 2011; Fitzpatrick et al., 2014; Fitzpatrick \& Pagani, 2012; Goldstein et al., 2013; Justice et al., 2011; Sabol \& Pianta, 2012a; Shaul \& Schwartz, 2014; Weiland \& Yoshikawa, 2013). When reviewing research based on children who are blind or visually impaired, articles found regarding kindergarten and school readiness focused exclusively on literacy and braille readiness. A search in educational databases including (EBSCO, Google Scholar and ERIC) with key words blind, kindergarten readiness, school readiness, preschool transition, braille, visually impaired, yielded few results. A search of the Journal of Visual Impairment and 
Blindness (JVIB), the seminal journal of the field, was also completed with similar results. The majority of articles using key words noted above produced articles relating to literacy, reading and braille readiness. In researching school readiness for children who are blind using education search sites yielded minimal peer reviewed articles within the past five years. A search of JVIB using key words including "readiness" "kindergarten" produced fifteen articles. Articles located focused on braille literacy, numeracy and concept development within the ECC but not addressing the whole child. A search of the literature specifically for school or kindergarten readiness and blindness yielded no current research in the areas of behavioral expectations, socialization, or self-concept or articles in alignment with early learner standards for sighted children. The following section will review research in the area of literacy and braille readiness. At the conclusion of this section, parent perspectives will be examined.

Braille and literacy development. Braille is a much more complicated means to access literacy than accessing the sighted word. Some researchers report early literacy development for children who are blind is in large regard similar to their sighted peers in addition to being distinct (Alper, 2012; Toussaint \& Tiger, 2010). Other researchers suggest less is similar and known about the development of language and literacy in children who are blind or visually impaired (Dodd \& Conn, 2000; Erickson \& Hatton, 2007; Steinman, LeJeune, \& Kimbrough, 2006). Yet most researchers are in agreement that children who are blind or have visual impairments are at risk of delays in acquisition of language and literacy skills compared to their sighted peers (Bigelow, 1987; Erickson \& Hatton, 2007; Fraiberg, 1977; Preisler, 1995; Pring, 1994; Urwin, 1984a). The following are developmental models proposed by Steinman, LeJeune, \& Kimbrough 
(2006), Pring, (1994) and Erickson and Hatton (2007) who have researched emergent literacy for children who are learning braille.

Steinman et al. (2006) made a compelling argument that Chall's (1983) model of reading stages applies to children learning braille. The period of school readiness is centered on the first three stages of Chall's (1983) model, where a child, either sighted or blind, is progressing through specific stages. Stage one involves pre-reading activities, exploring literacy and developing concepts, learning to distinguish letters, and letter approximations; stage two includes recognizing common words and exposure to print, letter shape and phonetic patterns and word recognition. Of Chall's (1983) six stages, the first three include learning to read, the last three include reading to learn. Steinman et al. (2006) highlighted the need for children who are blind and the need for direct, specific instruction from sighted adults. "In the pre-reading stage especially, children who are blind need to acquire a rich array of experiences that will prepare them to become competent readers" (Steinman et al., 2006, p. 41). Steinman et al. (2006) also identify braille readiness activities including instruction in the concept of spatial representation, tactile sensitivity in addition to auditory, tactile, conceptual and language abilities before a child learns to read. "Unlike their counterparts who are sighted, who learn language associations implicitly, pre-reading children who are blind require a larger amount of directed stimuli to establish accurate representations of the world" (Steinman et al., 2006, p. 42).

Pring (1994) identified a developmental model with crucial components in emergent braille including two phases of early literacy development, the logographic and alphabetic phases. The first phase is characterized by a sighted child recognizing words 
as whole units based on their visual appearance noting word length, double letters, and letter position (Barron \& Baron, 1977; Gathercole \& Baddeley, 1990; Pring, 1994; Seymour \& Elder, 1986; Snowling \& Frith, 1981). A sighted child demonstrates reading ability without instruction and knowledge in specific letter sound correspondence (Pring, 1994; Seymour \& Elder, 1986). The second phase, characterized by a child learning that letters represent sounds and that sounds represent letters and have a relationship to the spoken word (Pring, 1994). Pring (1994) also noted "The child who is blind experiences language as an auditory phenomenon in a much more exclusive way than a sighted child ever does" (Pring, 1994, p. 67). Pring summarized the ways in which braille and print reading differ as follows:

(a) Encoding strategy-with Braille, tactual input tends to be successive while with print, visual encoding of several letters may take place almost simultaneously; (b) accuracy-tactual acuity is significantly lower than that of vision and can resemble "blurred vision" (Apkarian-Stielau \& Loomis, 1975); (c) redundancy-because of the characteristics of the script, there is little redundancy in the Braille orthography, and therefore it is harder to read and requires more attention to the letter recognition processes than print reading (Pring, 1994); and (d) contractionsboth Braille and print use the alphabet, but in Braille shortened forms of letter clusters are employed to overcome the very real problems of reading speed. (Pring, 1994, pp. 68-69)

Despite these differences and challenges, children who are blind and use braille should have the same literacy expectations as their sighted peers.

Erickson and Hatton (2007) proposed using the conceptual framework developed 
by Sénéchal et al. (2001) to situate emergent literacy and young children with vision impairments. The need for a theoretical framework to guide research and practice (Erickson \& Hatton, 2007) is supported by the increasing interest and attention to the question of emerging literacy for preschool children who are blind. The conceptual framework proposed by Sénéchal et al. (2001) suggested emergent literacy included three key constructs: oral language, metalinguistic skills and print/literacy knowledge (Erickson \& Hatton, 2007; Sénéchal et al., 2001). Erickson and Hatton (2007) did not distinguish between early and emergent literacy: "Rather, we describe emergent and early literacy as a continuum of all the behavior, skills, processes, and concepts about written and oral language that precede and develop into conventional literacy skills" (p. 262). Three components of the theoretical model proposed by Sénéchal et al. (2001) are oral language, metalinguistic skills and print/literacy knowledge. Oral language includes narrative knowledge, vocabulary and knowledge of the world; metalinguistic skills include phonological awareness and syntactic awareness; print/literacy knowledge includes conceptual knowledge, functions of print, perception of self as a learner, emergent reading in context, procedural knowledge, phonetic spelling, alphabetic and letter-sound knowledge (Sénéchal et al., 2001). In seeking to support the use of Sénéchal et al. (2001) conceptual model, Erickson and Hatton (2007) culled data from a formal field study to support applicability of the model.

Three teachers of the visually impaired (TVI) who worked exclusively with children with visual impairment were participants in a formal study with Erickson and Hatton (2007). The researchers examined field notes, interviews and documents to include as data to support the applicability of the conceptual model. Erickson and Hatton 
cited research to support each of the three elements of the conceptual model then using data reported by the three teachers, identified the importance and relevance to their teaching practice.

Oral language. Researchers have cited concerns regarding language development for children who are blind (Fraiberg, 1977; MacComiskey, 1996; McConachie \& Moore, 1994; Stratton, 1996). Concerns include conceptual development, listening and attention, prolonged periods of echolalia, atypical use of pronouns and the extensive use of questions (Andersen et al., 1984; Bigelow, 1987; Pring, 1994; Urwin, 1984a). Some researches even suggest blind children do not have the concept that the spoken word is written down (Dodd \& Conn, 2000; Swenson, 1988).

Limited incidental learning and lack of access to nonverbal language cues including eye contact, gestures and facial expressions are factors that influence the delay of oral language for children who are blind (Erickson \& Hatton, 2007; Fraiberg, 1977; Preisler, 1995; Rogers \& Puchalski, 1984). Researchers suggested caregivers and teachers can support oral language development by providing child who are blind detailed and explicit descriptions of events, direct teaching of concepts and providing extensive real life experiences exposure to tangible objects (Warren \& Hatton, 2003; Wormsley \& D'Andrea, 1997).

Based on Erickson and Hatton's (2007) research, teachers understand the importance of oral language development and incorporate concepts into their instructional design. The teacher supported vocabulary development using direct teaching of objects, pre and post teaching concepts, use of modeling and rich descriptive language. They also incorporated oral language development into instruction when 
presenting story books and teaching comprehension.

Many of their interactions reflected the few suggestions in the literature of ways to enhance the development of oral language by young children with visual impairments, yet few of their interactions beyond repeated readings of a storybook involved print or it equivalent. All three teachers consistently used real-life objects and experiences to promote the development of vocabulary and concepts, thereby promoting oral language. (Erickson \& Hatton, 2007, p. 268)

Erickson and Hatton concluded from this data the field of education of the visually impaired should focus future research more explicitly on oral language and its relationship to early and emergent literacy.

Metalinguistic knowledge. Metalinguistic knowledge is phonological and syntactic awareness in a proposed model based on Sénéchal et al. (2001). This knowledge, specifically phonological knowledge has been the focus of research involving older children with visual impairments (Erickson \& Hatton, 2007). Yet "a comprehensive review of literature revealed no report of the metalinguistic skills of preschool-aged children with visual impairments" (Erickson \& Hatton, 2007, p. 268). What is clear from research is the need for phonological skill; in fact, phonological awareness and segmentation abilities seem to be a priority (Bradley \& Bryant, 1991; Dodd \& Conn, 2000; Pring, 1994). Braille contractions often cut across phonological boundaries causing more confusion for the child in the early stages of literacy development (Dodd \& Conn, 2000).

When describing school and braille readiness, metalinguistic development was not historically included (MacComiskey, 1996). Erickson and Hatton (2007), however, 
reported findings to suggest, "The recognition of letters in print or braille is not a prerequisite for the development of phonological awareness" (p. 268). Researchers reported preschool braille readers "were not able to identify a single letter presented in braille, but they achieved at least minimal success with segmenting syllables, isolating the initial sound in spoken words, and identifying non-rhyming words from a set of four" (Erickson \& Hatton, 2007, p. 269). Erickson and Hatton (2007) concluded children who are blind and learning braille do in fact develop phonological awareness without necessarily recognizing the braille alphabet. This is a critical observation for TVIs to continue to focus on emergent and early literacy even if a child is unable to progress accessing the code using tactile fine motor skills.

TVIs as a whole are not consistent in providing direct instruction in the area of metalinguistic knowledge, despite research that highlights the importance of these skills (Dodd \& Conn, 2000; Erickson \& Hatton, 2007). TVIs indirectly supported these skills by reading storybooks aloud, signing songs, chants, and rhymes. There was evidence of instruction that eventually would lead to phonological and syntactic awareness but few planned direct or indirect efforts to specifically support syntactic development (Erickson $\&$ Hatton, 2007). Lack of consistent instruction in syntactic concepts could further delay progression in writing as well as understanding contextual meaning from text. Erickson and Hatton (2007) concluded from this data the field of education of the visually impaired should focus future research more explicitly on metalinguistic knowledge in early and emergent literacy.

Conceptual and procedural literacy knowledge. Conceptual and procedural knowledge for a child reading braille is unique and distinct from reading readiness skills 
for their sighted peers. Children who are blind begin learning about braille and emergent literacy with tactile discrimination games to help develop skills to discriminate the dot patterns within the braille system. Tactile discrimination is more difficult than accessing the printed word. There are physical demands, unlike access to print, which require the child to sit still, control the position and movement of their hands and arms and maintain correct posture to read successfully with minimal tension and fatigue (Stone, 1988). Tactile discrimination of a word is one letter at a time whereas to visually access a word allows several letters to be identified simultaneously (Dodd \& Conn, 2000; Haber, Haber \& Furlin, 1983). In addition, tactual recognition is less accurate, "braille is perceived in moving, as opposed to static fixations of attention, the images fed to the brain tend to be incomplete and lacking in clarity, as evidence by the frequent occurrence of missed and added dot errors. If one dot is misperceived, then the letter is identified incorrectly" (Dodd \& Conn, 2000, p. 3). Reading braille is a literary activity yet also a tactile activity requiring fine motor discrimination, tactile skills and physical demands unlike accessing the printed word (Erickson \& Hatton, 2007; Lamb, 1996; MacComiskey, 1996). Lamb (1996) noted:

Reading is essentially a language task, so it is within this context that teachers need to investigate strategies and resources for fostering early braille skills. Therefore, early braille reading activities must be language based, and associated with meaningful text. To be relevant for the reading process, training in tactile perception must be concerned with language, must be meaningful tot he child, and should take into account the special skills required for reading by touch. (p. 186) Overall, research supports children who are blind have fewer opportunities to learn 
braille incidentally in early years and less direct experiences with the alphabet, braille or print, than their sighted counterparts (Craig, 1996; Erickson \& Hatton, 2007; Rex, Koenig, Wormsley, \& Baker, 1995; Swenson, 1999).

In my experience, as with the findings of Erickson and Hatton (2007), the tactile aspects of learning braille is the primary focus of direct teaching by TVIs in preschool and is the prominent role in educational curriculum and instruction. One teacher in the study identified "specific activities that were completed every week for the purpose of developing motor and tactile perception skills that would support the literacy skills that 'will come' later" (Erickson \& Hatton, 2007, p. 271). Another aspect of instruction supported in this area was use of the Perkins Braillewriter. Finally, all of the teachers reported curriculum and instruction that attempted to provide children with incidental and specific braille exposure activities, e.g. finding their names on a card, labeled cubbies, and accessible books (Erickson \& Hatton, 2007). Not surprisingly, Erickson and Hatton (2007) supported this as one of the elements in the conceptual framework for early and emergent literacy for students who read braille.

Given the complexity of learning a tactual code along with emergent literacy skills, limited literacy experience, limited access to braille texts, the nature of braille orthography and lack of incidental learning, it will take longer for the child who is blind to develop school readiness skills (Dodd \& Conn, 2000; Pring, 1984, 1994; Steinman et al., 2006). Employing the strategy of a Delphi Study, Koenig and Holbrook (2000) gained consensus from experts in the field of vision impairment, children who are emerging braille readers require a minimum of one hour per day in direct instruction with a TVI. There are limited resources to support this intensity of services which puts 
children who are blind at a disadvantage.

Mathematics and numeracy. For children who are sighted, the research in the area of mathematics and numeracy is far less than that of literacy.

Ignored for years in favour of a focus on literacy skills, children's early math skills are at least an equally important predictor of future school success as early literacy skills. Early math scores are not just predictive of later achievement in mathematics, they are almost as good a predictor of later reading as early reading scores are. (Farran, 2011, p. 7).

A thorough search of the literature resulted in no articles relating to kindergarten readiness and early mathematics and numeracy development for preschool children who are blind.

Social emotional school readiness. Preschool children with disabilities have a difficult time engaging in positive social interactions with their typical peers (Guralnick, Connor, Hammond, Gottman, \& Kinnish, 1996; McConnell \& Odom, 1999). Children with vision impairments are at greater risk of no engaging in positive social interactions, not displaying the full range of play behaviors and have reduced ability to gain entry into peer groups and resolve conflicts (Celeste, 2006; Sacks, Kekelis, \& Gayloard-Ross, 1992). "Studies have indicated that children who are visually impaired demonstrate play behaviors that are predominantly exploratory in nature. These children engage less frequently in manipulative play, or the functional use of toys, and demonstrate more stereotypical behavior during play (Celeste, 2006, p. 2). When a child who is blind is placed in a general education classroom without the necessary social competencies the result is isolation and limited peer interactions (Celeste, 2006). It is critical for children 
who are blind to learn social skills early to learn how to engage their peers and maintain positive relationships.

Toys and play are critical components of social emotional and behavioral instruction for school readiness. Playing board games, blocks, dramatic play and other social interactions are often key components of curriculum and instruction in the areas of pre-academics, cooperation, communication, persistence on task and cultural and social competence (Celeste, 2006; Perkins, Columna, Lieberman, \& Bailey, 2013; Rogers \& Puchalski, 1984). The difficulty however, is that most toys and curriculum are not adapted for children who are blind; they require adaptation, tinkering and alteration to meet the needs of the child who is blind (Alper, 2012).

Parental expectations and experience. Researchers note in general it is more stressful to raise a child with a disability, particularly a child who is blind (Ferrell, 1986; Leyser \& Heinze, 2001). Leyser and Heinze (2001) surveyed 130 parents of children who are visually impaired in the state of Illinois. Overall, parents were generally supportive of their child attending their neighborhood school (Leyser \& Heinze, 2001). With enrollment in public school, parents were concerned with social isolation, negative attitudes and misconceptions about blindness (Leyser \& Heinze, 2001). There was also a significant concern with lack of training for general education teachers and lack of daily access to teachers of the visually impaired (Leyser \& Heinze, 2001).

In early literacy development, most sighted parents take an active and primary role a child's pre-literacy activities and instruction. In contrast, most parents of children who are blind do not know braille and believe this is a barrier for them to help their child because they are unfamiliar with strategies and lack access to materials and experience 
with children who are blind (Alper, 2012). They believe they cannot help their child access the braille symbols, scaffold presentations or provide incidental interactions with braille without the assistance of a specialized teacher. Alper (2012) reported "Helping parents participate in their children's own reading by encouraging to learn braille themselves is a major outreach effort" (p. 248). It is important for TVIs to understand the critical role of the parent/caregiver in preparing their children for kindergarten and provide coaching and training so parents have the skills and confidence to support their child's learning.

\section{Ready schools}

In public schools there may be negative societal attitudes towards blindness and braille (Alper, 2012; Hehir, 2002). Hehir (2002) captured the concept of abelism toward children who are blind:

Reading Braille is a disability-specific method of reading that many nondisabled people view as unacceptable, preferring that children with very low vision read print even if they are inefficient readers due to their vision disabilities, and that totally blind children listen to tapes. (p. 10)

The educational system is often unable to ensure that students with disabilities have the same timely access to educational materials as their nondisabled peers (Alper, 2012). For the sighted, braille may be seen as cumbersome and costly, books take up a tremendous amount of space, transcription of printed material into braille is costly and time consuming and takes additional coordination between the general education teacher and the TVI. As an example, the braille edition of Harry Potter and the Deathly Hallows was the first popular children's novel to be distributed in both print and braille 
simultaneously. The braille version was 1,100 pages, weighed twelve pounds and contained twelve volumes (Alper, 2012; Oleck, 2010). Alper (2012) noted, "lack of accommodations for young children with visual impairments, such as materials supporting emerging literacy, ultimately impacts national productivity, poverty rates, and quality of life" (p. 245). While there have been many improvements to education for children who are blind and visually impaired, there is still much work to be done.

\section{Synthesis of Research}

After reviewing the literature regarding the construct and definition of school readiness from the perspectives of teachers (preschool, kindergarten, TVI), I found evidence of a need for a construct of readiness skills that specifically addresses children who are blind and use braille as their primary learning medium. Research has shown the importance of readiness skills for children who are sighted (Duncan et al., 2007; Farran, 2011; Kagan \& Rigby, 2003; Kern \& Friedman, 2009; Konold \& Pianta, 2005; Raver \& Knitzer, 2002; Weiland \& Yoshikawa, 2013; Workman et al., 2014; Xue \& Meisels, 2004). Furthermore, the research is more compelling regarding the need for solid skills as a child who is blind enters kindergarten and the impact long term on academic outcomes (Alper, 2012; Bigelow, 1990; Brennan et al., 2009; Celeste, 2006). The lack of research specific for children who are blind and readiness for kindergarten speaks to the significance and importance of this research. After a thorough review of the literature and using the Ecological and Dynamic Model of Transition (Rimm-Kaufman \& Pianta, 2000), school readiness for the purposes of this study will be compared to the developmental domains included in the Head Start Early Learning Outcomes Framework (USDHHS, 2015) identified by Oregon as early learning standards for children age three 
to five. Readiness skills are further clarified by the concepts assessed in the Oregon Kindergarten Readiness Assessment given to all incoming kindergarteners prior to beginning school.

\section{Critique of Research}

As demonstrated from the literature review, there is a gap in the research in regard to school readiness skills and children who are blind. While many authors highlight the need for further research in the area of braille or literacy readiness skills, there is no research that speaks to the construct framing the child in the center as the Ecological and Dynamic Model of Transition (Rimm-Kaufman \& Pianta, 2000).

A second factor that has prevented research in this area is the low incidence nature of blindness, specifically children who read braille. Arguably one of the most extensive research endeavors to date in the field of vision impairment was the Alphabetic Braille and Contracted Braille Study (ABC Braille Study) which focused on the acquisition of reading and writing skills by young children who were using braille as a primary learning medium (Emerson, Holbrook, \& D'Andrea, 2009; Holbrook \& Farrenkopf, 2009). The purpose of the study was to compare students who initially learned to read and write using contracted braille versus those who began with uncontracted braille (Emerson et al., 2009; Holbrook \& Farrenkopf, 2009). This was one of the largest studies in the field of vision impairments; participants included children from the United States and Canada who were identified with blindness and no other handicapping conditions. The total number of participants enrolled was 45 , a small sample size given the multinational effort. This does not reflect the efforts of the 
researchers but a reflection of the truly low incidence nature of vision impairment, specifically children who are blind and use braille as their primary medium.

In my review of the literature, I examined questions that related to the usefulness of identification of readiness skills for children who are blind and use braille as their primary learning medium. I highlighted areas of importance for teachers in preschool, kindergarten, and teachers of the visually impaired. I also highlighted importance for primary caregivers to have an identified construct of school readiness. Now, I review the methodologies noted in the research literature and best suited for my questions. My proposal is that hearing directly from TVIs and primary caregivers of children who are blind will identify their perspectives of school readiness that will help shape instructional focus and service delivery models for children who are blind entering kindergarten.

\section{Review of Methodological Literature}

In this section, I reviewed the methods best suited for identifying how TVIs and primary caregivers of children who are blind describe and explain school readiness. I reviewed the methodologies that best supported understanding the viewpoints, feelings and perceptions of TVIs and primary caregivers of children who are blind.

Creswell (2012) suggested qualitative research should be used when there is a problem or question that needs to be explored, when there are variables not easily measured. Denzin and Lincoln (2011) said in part "qualitative researchers study things in their natural settings, attempting to make sense of, or interpret, phenomena in terms of the meanings people bring to them" (p. 3). Qualitative researchers use the natural setting to collect data in the field where the problem is situated. They gathered information by talking directly to people within their natural context (Creswell, 2012). They want to 
hear directly from participants and minimize the power relationship that possibly occurs between the researcher and the participant (Creswell, 2012). Qualitative researchers collect data themselves through examining documents, observing or interviewing participants (Creswell, 2012). Qualitative researchers use multiple methods including surveys, interviews and document review (Creswell, 2012). Finally, qualitative researchers identify patterns and themes by organizing data to identify information directly from the research to formulate trends and meaning for suggestions for future practice (Creswell, 2012). According to Creswell (2012), "We use qualitative research to develop theories when partial or inadequate theories exist for certain populations and samples or existing theories do not adequately capture the complexity of the problem we are examining" (p. 48). For this research, I used a qualitative approach to data collection. The qualitative approach allowed me to learn directly from participants, allow identification of patterns and themes based on multiple forms of data including examination of permanent products and interviews. The qualitative method is without a doubt the most appropriate method to describe and explain how TVIs and primary caregivers construct the idea of kindergarten readiness for children who are blind and use braille as their primary learning medium.

\section{Justification of Selection of Methods}

Maxwell (2012) discussed the strength of qualitative research in relation to specific types of studies including understanding the meaning for participants, understanding contexts within which the participants act and the contextual influence, understanding the process by which events take place and identifying unanticipated phenomena and influences generating new theories or ideas. These strengths are useful 
for my study because they align with the theoretical framework and will allow for this research to be child-centered, taking into consideration the context and variables that surround them as they enter public school.

My intention is to provide guidance and information for TVIs and primary caregivers to better prepare children who are blind entering public school for the first time. Increasing understanding of readiness for school, improving service delivery in frequency and focus and supporting the home environment will possibly lead to increased academic outcomes for children who are blind. There is currently little research in the area of readiness skills and the perspective of TVIs and primary caregivers. My research sought to identify what these individuals currently do to facilitate readiness for children who are blind. TVIs and primary caregivers are intimately and extensively involved and influential in educational outcomes for children who are blind. They have the most information regarding what is currently happening, their insight should help guide improvement in educational practice.

The method of data collection used was an extensive document review to identify themes of current practice. This was followed by a semi-structured interview to verify themes identified with the primary contributors, TVIs and primary caregivers of children who are blind (Krathwohl, 2009; Mertens, 2010). I included open-ended questions regarding how TVIs and primary caregivers currently define and understand the construct of school readiness.

\section{Summary of Research Literature and Application to Study}

After a thorough review of the literature there is no question school readiness is a topic of interest to researchers in the field of education. Expectations for students 
entering kindergarten, both academically and behaviorally, have increased in recent years for all children, including those with disabilities. It will benefit children who are blind and improve educational practice to understand how TVIs and primary caregivers understand and operationalize the construct of school readiness in preparing children who are blind for the kindergarten classroom. 


\section{Chapter 3: Methods}

In this chapter, I review the problem and purpose of this study and present a description of methods to answer the primary research question: How is the construct of school readiness defined for children who are blind and use braille as their primary medium? I elaborate on the methods, participants, as well as procedures and instruments, data collection and analysis used in this research. Next, I present possible biases and limitations of the methods involved in this study. Finally, I conclude this chapter by explaining procedures to protect the confidentiality of participants.

\section{Research Methods}

In the following section I review the statement of the research problem and purpose of this research followed by a presentation of the research design, paradigm guiding inquiry. Also presented are the procedures, data collection and analysis including instruments and measures, data analysis, and role of the researcher.

\section{Statement of the Research Problem}

School readiness for children who are blind and use braille as their primary learning medium has been primary focused on braille literacy. Therefore, teachers of the visually impaired (TVI) and primary caregivers can lack the background and knowledge to fully prepare students who are blind for the general education setting. The Ecological and Dynamic Model of Transition (Rimm-Kaufman \& Pianta, 2000) is a framework applicable for students who are blind and using braille as they prepare and transition from early childhood education to kindergarten because if students are not fully prepared to transition to kindergarten barriers may exist to fully access opportunities in the general education kindergarten. The framework is also applicable in addressing the lack of 
research for kindergarten readiness outside of braille readiness for children who are blind. Additional components of kindergarten readiness must be examined and addressed because children who are blind entering kindergarten have additional and more complex needs than their sighted peers. This research is based on the need to know more about how to prepare children who are blind and use braille for public school.

\section{Purpose of Research}

The purpose of this research was to describe and explain how TVIs and primary caregivers define the construct of kindergarten readiness for children who are blind and use braille as their primary learning medium. The primary research question is how is the construct of school readiness defined for children who are blind and use braille as their primary medium? Additional research questions included: (a)What is the current focus of instruction for children who are blind in preschool to prepare them for kindergarten? (b) What is the role of the TVI and primary caregiver in preparing students for kindergarten? (c) What do TVIs and primary caregivers identify as the barriers to school readiness for children who are blind? and (d) What do TVIs and primary caregivers see as essential to preparing children who are blind for kindergarten?

Understanding current constructs of school readiness from the perspective of TVIs and primary caregivers was studied by examining key documents and interviewing key participants. The documents examined were Individualized Family Service Plans (IFSP) and Individual Education Plans (IEP) for ten students in preschool and entering/exiting kindergarten. The goals and objectives identified in these documents were analyzed with the Head Start Early Learning Outcomes Framework (USDHHS, 2015) to compare expectations for children who are blind with school readiness expectations for children 
who are sighted. Goals and objectives were also analyzed with the Expanded Core Curriculum (Hatlen, 1996) that identifies skills and competencies specifically for children who are blind or visually impaired. These findings may provide a foundation to assess the type and frequency of services students received in early childhood special education and ascertain the current understanding of what it means to be ready for kindergarten for students who are blind and use braille as their primary learning medium.

\section{Type of research design and rationale}

Qualitative methods were used to answer the research question of: How is the construct of school readiness defined for children who are blind and use braille as their primary medium? Strengths of qualitative research are: understanding the meaning for participants; understanding contexts within which the participants act and the contextual influence; understanding the process by which events take place; and identifying unanticipated phenomena and influences generating new theories or ideas (Maxwell, 2012). These methodological strengths align with the theoretical framework of the Ecological and Dynamic Model of transition (Rimm-Kaufman \& Pianta, 2000), and allow this research to be child-centered, taking into consideration the context and variables that surround children who are blind as they enter public school. In addition, due to the low incidence nature of children who are blind and use braille, a quantitative large group research design would not suit the purpose of this research.

My research has a basis in Grounded Theory developed by Corbin and Strauss (1994), which has been described as "a general methodology for developing theory that is grounded in data systematically gathered and analyzed" (p. 273). I used artifacts including IFSPs and IEPs to identify themes of current practice. I then sought to verify 
my findings from these artifacts based on themes that emerged from these IFSP and IEP artifacts through semi-structured interviews with TVIs and primary caregivers.

My hypothesis was that education services for children who are blind and use braille, both the type and intensity needed, do not align with the current construct of school readiness for sighted children based on the Head Start Early Learning Outcomes Framework (USDHHS, 2015) which was used in part as a guide to develop the Oregon Kindergarten Readiness Assessment. Children, who are blind, in some instances, can be perceived by general education teachers as needing extensive supports to participate in general education and therefore, the general education classroom is not seen as an appropriate educational placement (Alper, 2012; Hehir, 2002). If children who are blind have the necessary school readiness skills including academic and social skills when entering kindergarten, they will be better prepared to participate fully in the academic classroom and placement in the general education classroom.

To reiterate, the primary research question is: How is the construct of school readiness defined for children who are blind and use braille as their primary medium? Additional research questions included: (a) What is the current focus of instruction for children who are blind in preschool to prepare them for kindergarten? (b) What is the role of the TVI and primary caregiver in preparing students for kindergarten? (c) What do TVIs and primary caregivers identify as the barriers to school readiness for children who are blind? and (d) What do TVIs and primary caregivers see as essential to preparing children who are blind for kindergarten?

In the first phase of this research, students' IFSP and IEP documents were examined to address the following questions: (a) What are the current educational 
services for children who are blind and use braille as their primary learning medium? (b) What types of intervention and education are children ages three to five receiving? (c) Do these families continue to receive support during this time? (d) What type of supports do students and families receive? (e) What are the specific goals and objectives on the IFSP and IEP documents? (f) How are the IFSP and IEP goals aligned with the Head Start Early Learning Outcomes Framework (USDHHS, 2015) and the Expanded Core Curriculum (Hatlen, 1996)?

In the second phase of this research, I conducted semi-structured interviews with TVIs, and primary caregivers of children who are blind. Questions were formed in part by results from phase one of this research and included the following: (a) What were your priorities for readiness in kindergarten? (b) What skills did you see as supporting successful transition for your child in the typical classroom? (c) What were the barriers for your child attending a typical kindergarten?

\section{Paradigm guiding inquiry}

This research was grounded in social constructivism, where individuals seek to understand the world in which they live using a holistic view of the individual and the many factors and context that influence and impact their existence (Creswell, 2013). Research questions generally start out broad and general so that participants can construct the meaning of the situation. Use of an inductive method of emergent ideas (through consensus) obtained through methods such as interviewing, observing, and analysis of texts" (Creswell, 2013, p. 36). In my research, I was interested in the views and voice of the participants. My intention was to understand their construction of the notion of school readiness and build upon their understanding to guide educational practice and 
increase school outcomes for students who are blind. Thus, I used a qualitative method grounded in social constructivism to situate my research.

\section{Participants Phase One}

For phase one of the study, a document review was conducted that analyzed two IFSPs and incoming and outgoing kindergarten IEP documents for children who met the following criteria: (a) identified as eligible and receiving services for vision impairment under the Individuals with Disability Education Act (IDEIA) and Oregon eligibility, (b) identified as tactile learners using braille as their primary learning medium, and (c) were currently in sixth grade or earlier. I requested documents from all eight regional programs in Oregon with educational programs for children who are blind or visually impaired (See Appendix A). Two of the 10 students had only one IEP during the kindergarten year. I also asked that all identifying information be redacted before the documents were sent. A total of 10 students' documents from four of the eight regional programs were collected and analyzed for phase one. Of the 10 student documents analyzed, eight were female students, two were male, eight only had a special education eligibility of vision impaired; one had a secondary disability of communication disorder, one had a secondary eligibility of hearing impaired. All 10 students attended a formalized preschool placement, three Head Start classroom four days per week, two in community preschool, two days per week, and five received services in an ECSE classroom, two days per week. Table 3.1 is a summary of student participants. The following special education IDEIA codes are used: 40 vision impairment, 50 communication disorder, 20 deaf or hard of hearing, and 43 for deaf blind. For related services the following abbreviations are used: OM for orientation and mobility, OT for 
SCHOOL READINESS: CHILDREN WHO ARE BLIND

occupational therapy, PT for physical therapy, and SLP for speech/language pathology

services.

Table 3.1

Summary of Student Participants

\begin{tabular}{|c|c|c|c|c|c|c|c|}
\hline Student & $\begin{array}{l}\mathbf{M} \\
/ \\
\mathbf{F}\end{array}$ & $\begin{array}{c}\text { Primary } \\
\text { Disability }\end{array}$ & $\begin{array}{c}\text { Other } \\
\text { Disability }\end{array}$ & $\begin{array}{l}\text { Related } \\
\text { services: } \\
\text { Preschool }\end{array}$ & $\begin{array}{c}\text { Related } \\
\text { services: } \\
\text { Kinder. }\end{array}$ & $\begin{array}{l}\text { Preschool } \\
\text { placement }\end{array}$ & $\begin{array}{l}\text { Kinder. } \\
\text { Place- } \\
\text { ment }\end{array}$ \\
\hline 1 & $\mathrm{~F}$ & 40 & None & $\begin{array}{l}\text { OM (Year } \\
\text { one only) }\end{array}$ & $\mathrm{OM}$ & $\begin{array}{c}\text { Integrated ECSE } \\
\text { Classroom }\end{array}$ & $\begin{array}{l}\text { General } \\
\text { Ed. } \\
5 \% \\
\text { removal }\end{array}$ \\
\hline 2 & $\mathrm{~F}$ & 40 & None & $\begin{array}{l}\text { OT, PT, } \\
\text { SLP }\end{array}$ & $\mathrm{OM}$ & ECSE Preschool & $\begin{array}{l}\text { General } \\
\text { Ed. } \\
20 \% \\
\text { removal }\end{array}$ \\
\hline 3 & $\mathrm{~F}$ & 40 & None & $\mathrm{OM}$ & $\mathrm{OM}$ & $\begin{array}{l}\text { Community } \\
\text { Preschool }\end{array}$ & $\begin{array}{l}\text { General } \\
\text { Ed. } \\
20 \% \\
\text { removal }\end{array}$ \\
\hline 4 & M & 40 & 50 & $\begin{array}{l}\text { OM, SLP, } \\
\text { OT, PT }\end{array}$ & OT, OM & Head Start & $\begin{array}{l}\text { Structure } \\
\text { Learning } \\
\text { Center } \\
66 \% \\
\text { General } \\
\text { Ed. } 34 \%\end{array}$ \\
\hline 5 & $\mathrm{~F}$ & 40 & None & SLP, OM & $\mathrm{OM}$ & ECSE Preschool & $\begin{array}{l}\text { General } \\
\text { Ed. } \\
20 \% \\
\text { removal }\end{array}$ \\
\hline 6 & M & 40 & $20,50,43$ & $\begin{array}{c}\text { SLP, } \\
\text { Audiology }\end{array}$ & $\begin{array}{c}\text { OT, } \\
\text { Audiology }\end{array}$ & ECSE Preschool & $\begin{array}{l}\text { General } \\
\text { Ed. } \\
33 \% \\
\text { removal }\end{array}$ \\
\hline 7 & $\mathrm{~F}$ & 40 & 50 & None & OM, SLP & Head Start & $\begin{array}{l}\text { General } \\
\text { Ed. } \\
20 \% \\
\text { removal }\end{array}$ \\
\hline
\end{tabular}


(Continued)

\begin{tabular}{|c|c|c|c|c|c|c|c|}
\hline Student & $\begin{array}{l}\mathbf{M} \\
/ \\
\mathbf{F}\end{array}$ & $\begin{array}{c}\text { Primary } \\
\text { Disability }\end{array}$ & $\begin{array}{c}\text { Other } \\
\text { Disability }\end{array}$ & $\begin{array}{c}\text { Related } \\
\text { services: } \\
\text { Preschool }\end{array}$ & $\begin{array}{c}\text { Related } \\
\text { services: } \\
\text { Kinder. }\end{array}$ & $\begin{array}{l}\text { Preschool } \\
\text { placement }\end{array}$ & $\begin{array}{l}\text { Kinder. } \\
\text { Place- } \\
\text { ment }\end{array}$ \\
\hline 8 & $\mathrm{~F}$ & 40 & None & None & None & $\begin{array}{c}\text { Integrated ECSE } \\
\text { Classroom }\end{array}$ & $\begin{array}{l}\text { General } \\
\text { Ed. } \\
20 \% \\
\text { removal }\end{array}$ \\
\hline 9 & $\mathrm{~F}$ & 40 & None & $\begin{array}{l}\text { OT, PT, } \\
\text { OM }\end{array}$ & OT, PT, OM & $\begin{array}{l}\text { Community } \\
\text { preschool }\end{array}$ & $\begin{array}{l}\text { Structure } \\
\text { Learning } \\
\text { Center } \\
75 \% \\
\text { General } \\
\text { Ed. } 25 \%\end{array}$ \\
\hline 10 & $\mathrm{~F}$ & 40 & None & None & $\mathrm{OM}$ & Head Start & $\begin{array}{l}\text { General } \\
\text { Ed. } 20 \% \\
\text { removal }\end{array}$ \\
\hline
\end{tabular}

Note. Primary disability: $40=$ vision impaired; $50=$ communication disorder; $20=$ hearing impaired; $43=$ deaf blind. Related service provider: SLP = speech/language pathologist; OT = occupational therapist; PT

$=$ physical therapist; $\mathrm{OM}=$ orientation and mobility specialist.

\section{Participants Phase Two}

In phase two, I interviewed five TVIs and three primary caregivers who participated in educational programs for the visually impaired in Oregon.

I invited all TVIs in the state to participate in the interview process. There are approximately 80 TVIs in Oregon, an email was sent through the state list serve. I asked TVIs to participate by sending a letter (see Appendix B) through the Oregon email listserv asking for voluntary participation. Participants self-selected by contacting the researcher to schedule an interview at the participants' convenience. I conducted interviews using face-to-face and telephone. All primary caregivers were via telephone and used the same core questions. The different format occurred when interviewing the TVIs, three via face to face; two via telephone. The different interview formats were similar in that I began by explaining my study, assuring anonymity, and asking consistent 
questions, allowing the interviewee to guide the semi-structured interview. The total of five TVIs interviewed represented approximately $6 \%$ of TVIs in Oregon.

Due to the low incidence nature of this population, purposive sampling yielded the most participants. Participants were not randomly selected; they were selected based on their position as a TVI or primary caregiver in Oregon. The intent of this research was not to generalize to a larger population but to understand more intimately the construct of readiness for children who are blind in Oregon. TVIs and primary caregivers of children who are blind were uniquely positioned to guide research into school readiness for this population of children.

Primary care givers were selected by asking TVIs to distribute the letter directly to primary caregivers in their region for children who fit the stated criteria (See Appendix E). I did not have direct access to contact information and it would be a violation of confidentiality for TVIs to give me contact information from their regions.

Teachers of the visually impaired. A total of five TVIs were interviewed. TVIs are teachers certified through Oregon Teacher Standards and Practice Commission to teach children with vision impairments birth through age 21 . This is a unique license in that many TVIs are licensed to serve children and families birth through age five but the majority of university programs only provide one class for this specific population. The majority of TVIs are not licensed early childhood specialists, however, some have specialized in the Early Childhood Special Education (ECSE) population. TVIs included those who worked exclusively in ECSE as well as those who taught school age students including kindergarten. I assured TVIs their responses were confidential and did not contain identifying information. They also could refuse to answer any questions and 
revoke consent at any time. The five TVIs interviewed included two men and three women, the range of years in the field was from ten years to thirty with an average of 18 years in the field of vision impairment. Three out of the five TVIs were also certified Orientation and Mobility Specialists.

Primary caregivers. A total of three primary caregivers were interviewed. Recruitment occurred through TVIs in the state and was voluntary. I asked TVIs to give a letter (see Appendix C) to primary caregivers explaining the research and inviting their participation; primary caregivers voluntarily contacted me. Primary caregivers were assured that all information was confidential and that no identifying information was included in this research. I informed primary caregivers that they could refuse to answer any questions and revoke consent at any time. Of the three primary caregivers, all were biological mothers with first born children born blind; one was the mother of twins, one sighted, and one blind.

\section{Procedures}

The procedures for this study began based upon approval from the Portland State University Human Subjects Committee. The following is a description of the two phases of this research.

\section{Phase one: Document Review}

Documentation review establishes an impression of how a program operates without disrupting the program (Mertens, 2010). The advantages of document review included being able to obtain comprehensive and historical information, there was no disruption to the program or routine, the information already existed, and there were few biases about the information (Mertens, 2010). The disadvantage was it took time to 
access and examine information or information may be incomplete or restricted (Mertens, 2010).

I measured the construct of school readiness for children who are blind and use braille starting with an extensive document review using the Ecological and Dynamic Model of Transition (Rimm-Kaufman \& Pianta, 2000) to guide viewing the child within their natural context. The Head Start Early Learning Outcomes Framework (USDHHS, 2015) was used as a framework to examine the artifacts; as stated earlier, this framework guided the development of the Oregon Kindergarten Readiness Assessment. When a child is blind, they receive early intervention services, an IFSP is developed at age three through age five at age five they transition to an IEP and transition to kindergarten. For each participant I examined the two IFSP from age three through five as well as the two IEP at the beginning and end of their kindergarten year.

IFSP and IEP are extant texts, documents that already existed as opposed to elicited ones. Extant texts "require that the researcher be aware of the need to temper their use with an understanding of the time, context and intended use for which the materials were created" (Mertens, 2010, p. 373). As the researcher, I remained aware the IFSP and IEP documents were the result of a team process and no one professional or the primary caregiver had sole responsibility or control in the process of determining priorities and services. I did not interact with the people who created the documents for review.

I obtained confidential documents through a request to administrators and coordinators for education programs for visually impaired children in Oregon. I requested all identifying information be redacted to ensure anonymity to protect the 
identification of the child, primary caregiver and TVI. Student data sets included IFSP and IEP documents from age three through kindergarten. For the purposes of this study I examined 10 student data sets.

During phase one, I examined and coded artifacts through the process of initially categorizing the data into codes based on the Head Start Early Learning Outcomes Framework (USDHHS, 2015) and the Expanded Core Curriculum (Hatlen, 1996). In qualitative research, coding refers to a word or short phrase that captures a salient, summative, statement that captures the essence of data (Saldana, 2013). Saldana (2013) stated "Coding is a heuristic (from the Greek, meaning 'to discover') an exploratory problem-solving technique without specific formulas or algorithms to follow" (p.8). Coding is not simply labeling, it is leads from data to themes and ideas (Richards \& Morse, 2007). In this initial phase, a technique of qualitative content analysis (Cho \& Lee, 2014; Moretti et al., 2011; Sandelowski, 2000) was used to classify IFSP and IEP goals and objectives into identified categories. To complete the coding process an examination of the specific goal or objective was correlated with a specific domain and sub domain of the Head Start Early Learning Outcome Framework (USDHH, 2015) (see Table 1.1) and the Expanded Core Curriculum (Hatlen, 1996) (see Table 1.2). The Head Start Early Learning Outcome Framework (USDHH, 2015) provided specific examples of educational outcomes for each sub domain of the framework; this allowed a comparison of the goals and objectives with the framework.

Inter-observer reliability was completed with two colleagues who are TVIs to validate findings. Through this comparison, I had the opportunity to articulate my thinking process and presented an opportunity to clarify emergent ideas and insights from 
the data. To address reliability of coding of documents, two TVIs coded three student data sets $(30 \%)$. Each TVI has been working in the field for over 15 years and are both certified as Orientation and Mobility Specialists. Prior to coding, I familiarized them with the Head Start Early Learning Outcome Framework (USDHHS, 2015); they already have an expertise with the Expanded Core Curriculum (Hatlen, 1996). Table 3.2 is a summary of the inter-observer reliability agreements based on Head Start Early Learning Outcome Framework (USDHHS, 2015).

Table 3.2

Summary Inter-Observer Reliability Agreement: Head Start Early Learner Outcomes Framework (USDHHS, 2015).

\begin{tabular}{lllll}
\hline Student & Total N & TVI 1 & TVI 2 & Agreement \% \\
\hline 1 & 30 & $27 / 30$ & $28 / 30$ & $90 ; 90$ \\
2 & 42 & $36 / 42$ & $37 / 42$ & $86 ; 88$ \\
3 & 26 & $25 / 26$ & $25 / 26$ & $96 ; 96$ \\
\hline
\end{tabular}

Table 3.3 is a summary of the inter-observer reliability agreement for the Expanded Core Curriculum (Hatlen, 1996).

Table 3.3

Summary Inter-Observer Reliability Agreement: Expanded Core Curriculum (Hatlen, 1996).

\begin{tabular}{lllll}
\hline Student & Total N & TVI 1 & TVI 2 & Agreement \% \\
\hline 1 & 30 & $28 / 30$ & $25 / 30$ & $93 ; 83$ \\
2 & 42 & $25 / 42$ & $26 / 42$ & $60 ; 62$ \\
3 & 26 & $19 / 26$ & $18 / 26$ & $73 ; 69$ \\
\hline
\end{tabular}

Percentages are lower for the Expanded Core Curriculum (Hatlen, 1996) versus the Head Start Early Learner Outcomes Framework (USDHHS, 2015). This may be due to the 
broader categories of the ECC and that often context determines which area a goal or objective may be addressing. For example, matching sound containers could be compensatory skills learning same and different or sensory efficiency, identifying sounds or a pre-skill for orientation and mobility.

\section{Phase two: Semi-structured interviews}

The second phase of this research allowed me to identify themes through the use of semi-structured interviews. Interviews allowed the voices of teachers and primary caregivers to be heard. Mertens (2010) asserted, interviews are used when "you want to fully understand someone's impressions or experiences" (p. 352). The advantage of interviews was to obtain a full range and depth of information; to truly hear participants' views and allows for the participant to have an active role in the research. The disadvantages could be the time it takes to complete interviews, results could be hard to analyze and compare, be costly, or the interviewer could bias the participant's responses (Mertens, 2010). These disadvantages were mitigated by completing interviews via phone or face-to-face when participants were in close proximity, using in vivo coding and asking for participants on a voluntary basis for a short period of time, no more than 20 minutes. Interviews were particularly beneficial for my research because it allowed me to: (a) explore the current construct of school readiness from the perspective of the TVI and primary caregiver, (b) determine how TVIs and primary caregivers perceive and construct meaning around school readiness (c) determine what is significant or less important to them regarding school readiness and (d) determine how or if they thought change would lead to better outcomes for students who are blind and use braille as their primary medium. 
An introductory meeting was scheduled with interview participants to share the purpose of the research, discuss confidentiality, and get assurances from TVIs and primary caregivers that wanted to participate (Mertens, 2010). I asked open-ended questions to allow the participants to respond (See Appendices D \& E). The interviews included questions around a child's experiences in education prior to entering kindergarten, opinion and/or value questions regarding the readiness of the child to enter kindergarten as well as knowledge and background questions around services available, options for trainings, professional development, and parent support groups. I completed three interviews with primary caregivers and five interviews with TVIs to increase accuracy of results (Mertens, 2010).

Interview questions were developed based on the literature review and themes identified from examination of student data sets and followed a semi-structured interview protocol. Interviews and interview questions ranged from highly structured with a rigorous set of questions to unstructured following more of a conversational format (Kvale, 2008; Vogt, Gardner, \& Haeffele, 2012). Semi-structured interviews were openended with a structured component but allowed new ideas to be brought up during the interview as a result of how the interviewee responded (Kvale, 2008; Vogt et al., 2012). Kvale (2008) described a quality interview as one that allowed for spontaneous answers from the interviewee with the interview being "self-communicating" in that a story emerges through the process. Semi-structured interviews sought to address the primary research question: How is the construct of school readiness defined for children who are blind and use braille as their primary medium? Additional research questions included: (a) What is the current focus of instruction for children who are blind in preschool to 
prepare them for kindergarten? (b) what is the role of the TVI and primary caregiver in preparing students for kindergarten? (c) What do TVIs and primary caregivers identify as the barriers to school readiness for children who are blind? (d) What do TVIs and primary caregivers see as essential to preparing children who are blind for kindergarten?

During phase two, I recorded and coded interviews line by line. "Line by line coding frees you from becoming so immersed in your research participants' world-views that you accept them without question" (Charmaz, 2014). This type of coding is particularly critical when researchers from professional fields are studying members from their respective profession (Charmaz, 2014). I used in vivo coding: the codes adopted directly from the Head Start Early Learning Outcome Framework (USDHHS, 2015) and interview data. I relied on memo writing throughout the research as a way to analyze ideas about the data and codes.

\section{Data Collection and Analysis}

The following is a presentation of data collection and analysis including presentation of instruments and measures, data analysis and role of the researcher.

\section{Instruments and measures}

Data collection included an extensive document review of student IFSP and IEP documents. Collection of documents resulted in a complete student data set of IFSP and IEPs from age three through kindergarten. A review of the student data set was chosen as the instrument and measure; I used results form the phase one to form interview procedures during phase two. Examining student data sets from pre-formal schooling and the first year of formal schooling helped identify the focus of instruction during the time of transition to kindergarten. This examination also identified alignment with the Head 
Start Early Learning Outcomes Framework (USDHHS, 2015) and the Expanded Core

Curriculum (ECC) for blind and visually impaired students (Hatlan, 1996). Table 3.4 is

a brief sample of student data, Appendix F has a complete data analysis of one student's documents.

Table 3.4

Sample Student Data

\begin{tabular}{|c|c|c|c|c|c|}
\hline Preschool 1 & Preschool 2 & Incoming K & Outgoing K & $\begin{array}{l}\text { HS Domain/ } \\
\text { Subdomain }\end{array}$ & ECC Area \\
\hline $\begin{array}{l}\text { Read } 15 \\
\text { letters }\end{array}$ & $\begin{array}{l}\text { Read } 15 \\
\text { letters }\end{array}$ & $\begin{array}{l}\text { Demonstrate braille } \\
\text { readiness: spatial } \\
\text { position of dots; track } \\
\text { a line of dots; explore } \\
\text { braille page; locate } \\
\text { name in braille; } \\
\text { identify upper \& } \\
\text { lower case letters; } \\
\text { read } 21 \text { of } 21 \text { whole- } \\
\text { word alphabet words; } \\
\text { read } 6 \text { of } 6 \text { whole \& } \\
\text { part word } \\
\text { contractions. }\end{array}$ & $\begin{array}{l}\text { Demonstrate } \\
\text { knowledge and } \\
\text { proficiency in } \\
\text { reading and writing } \\
90 \% \text { of braille } \\
\text { code, alphabet, } \\
\text { numbers, } \\
\text { punctuation, } \\
\text { composition, signs, } \\
\text { and alphabet } \\
\text { contractions } \\
\text { including short } \\
\text { form words. }\end{array}$ & $\begin{array}{l}\text { Literacy: } \\
\text { Print and } \\
\text { alphabet } \\
\text { knowledge }\end{array}$ & $\begin{array}{l}\text { Compensatory } \\
\text { or functional } \\
\text { academic } \\
\text { skills, } \\
\text { including } \\
\text { communication } \\
\text { modes }\end{array}$ \\
\hline
\end{tabular}

I additionally selected an interview method to understand further the current construct of readiness skills from the perspective of key stakeholders in the child's life. This allowed me to identify themes such as how important is the construct of readiness for TVIs and primary caregivers and, what are the most important skills for children who are blind as they enter kindergarten from the perspective of the TVI, and primary caregiver.

All data were stored and maintained in a locked file cabinet. All documents and information were confidential and secured to ensure anonymity of the participants. All data and information will be stored for a period of three years after the completion of this study. 


\section{Data Analysis}

Analysis of the student data collected in phase one involved a comparison between goals and objectives identified on the IFSPs and IEPs and the following Head Start Early Learning Outcomes Framework (USDHHS, 2015) preschool domains (Table 1.1): Approaches to Learning, Social and Emotional Development, Language and Communication, Literacy, Mathematics Development, Scientific Reasoning and Perceptual, Motor, and Physical Development. In addition, an analysis was completed that compared goals and objectives to the ECC (Hatlen, 1996) specific for children with visual impairments including: Compensatory or Access Skills, Career Education, Independent Living Skills, Orientation and Mobility, Recreation and Leisure Skills, Social Interaction, Self-Determination Skills, Assistive Technology, and Sensory Efficiency (Hatlen, 1996). A total of 10 student data sets were collected and analyzed for this research.

In analyzing the interview data obtained in phase two, I used memo writing during the interview to capture ideas in the midst of the interview (Charmaz, 2014). After interviews were complete and transcribed, I used a line-by-line coding process for initial coding. Next, a focused selective process of coding was used that identified the most significant and/or frequent initial codes to sort, synthesize, integrate, and organize large data from interviews (Charmaz, 2014).

\section{Role of the Researcher}

As a researcher, I am positioned in the research by my role as the Senior Director of Columbia Regional Program, a program that educates 250 children with vision impairments in Oregon. I am also a certified TVI and have worked in the field of vision 
impairment for over 20 years. When collecting, interviewing, and analyzing data I disclosed my position and background to the participants. I acknowledged that I have bias in that as a TVI I have opinions regarding professional practice. As an administrator of a program for blind/visually impaired children, my role had been to evaluate teachers and their practice. I acknowledged to participants providing student document sets as well as interviewees my bias, influence, and my perceptions in the results of this research.

When conducting the interview with TVIs, I had insider status as the researcher (Bassett, Beagan, Ristovski-Slijepcevic, \& Chapman, 2008). I had richer knowledge of this group by my lived experiences. I used caution to not put myself in a place of power as an administrator and acknowledged and eliminated any risk to participants. I accomplished this by assuring TVIs that results and information would be confidential and presentation of results would ensure anonymity. When I interviewed primary caregivers, I remained neutral and committed to having meaningful dialogue, and to place control of the research agenda in their hands (Mertens, 2010). I was able to mitigate my outsider status by acknowledging my experience with children who are blind and finding other commonalities with participants such as being a parent and our common interest in children's success in education. 


\section{Chapter 4: Results/Analysis}

In this chapter, I review the purpose of this research and present an analysis of the results aligned with the research questions as well as identification of study limitations. The purpose of this research was to describe and explain how TVIs and primary caregivers construct the idea of kindergarten readiness for children who are blind and use braille as their primary learning medium. The primary question was: How is the construct of school readiness defined for children who are blind and use braille as their primary medium? I identified current understanding and constructs of school readiness from the perspective of TVIs and primary caregivers of children who are blind through the review of existing documents and interviews with participants. Understanding current constructs of school readiness by examining key documents and interviewing key participants allowed a comparison of school readiness expectations for children who are sighted and those who are blind. While children who are blind are unique and have unique educational needs, these findings provide a foundation to assess the type and frequencies of services students received in early childhood special education and ascertain the current understanding of what it means to be ready for kindergarten if you are blind. Additional research questions included: (a) What is the current focus of instruction for children who are blind in preschool to prepare them for kindergarten? (b) what is the role of the TVI and primary caregiver in preparing students for kindergarten? (c) What do TVIs and primary caregivers identify as the barriers to school readiness for children who are blind? (d) What do TVIs and primary caregivers see as essential to preparing children who are blind for kindergarten? 


\section{Analysis of Data}

The following is a presentation of the analysis of the data based on the research. This research was completed in two phases, phase one document review is analyzed followed by an analysis of phase two, semi-structured interviews.

\section{Phase one}

As previously stated, 10 student data sets were gathered from four regional programs in Oregon. All data sets included two IFSPs from two years in preschool and IEPs during the kindergarten school year for each student. As each student data set was analyzed, the goals and objectives were coded based on the Head Start Early Learner Outcomes Framework (USDHHS, 2015) and the Expanded Core Curriculum (Hatlen, 1996) for blind and visually impaired children. Each data set was analyzed and coded, a journal was kept to organize memos and field notes and identify trends, concepts, and ideas to refine future interview questions for participants.

\section{Phase Two}

As previously stated, an email (Appendix B) was sent through the Oregon TVI listserv to request participation of TVIs in an interview process. Five teachers of the visually impaired were representing three regional programs in Oregon volunteered to participate in the interview process. An email (Appendix C) was sent to TVIs to ask primary caregivers to participate in an interview process. This method yielded two parents who were initially willing to participate; both subsequently declined to participate citing lack of time. Three primary caregivers from one region were interviewed who had children who were identified as using braille as their primary medium. These primary caregivers were selected from the TVIs in the region. Each interview was semi- 
structured with the participant able to guide the conversation. Interviews varied in length from 12 to 29 minutes, each was recorded and transcribed. Through the initial coding process, data was analyzed to make sense of the participants' stories. Using a Grounded Theory (Corbin \& Strauss, 1994) strategy, the researcher used memo writing during the interview to capture ideas in the midst of the interview (Charmaz, 2014). After interviews were complete and transcribed, I used a line-by-line coding process for initial coding. Interview responses were coded initially based on the primary research question as well as additional questions of this study. Next, a focused selective process of coding was used that identified the most significant and/or frequent initial codes to sort, synthesize, integrate, and organize large data from interviews (Charmaz, 2014).

\section{Presentation of Results}

The following is a presentation of results from phase one and two of this research.

\section{Phase One}

Phase one was an extensive documents review, specifically comparing IFSP and IEP documents to the Head Start Early Learning Outcome Framework (USDHHS, 2015) and the Expanded Core Curriculum (Hatlen, 1996).

Head Start Early Learning Outcomes Framework. The research began with analysis of student data sets by coding goals and objectives based on the Head Start Early Learning Outcomes Framework (USDHHS, 2015) domains and sub-domain (see Table 1.1 for complete list of domains and subdomains). Table 4.1 includes quantitative data that resulted in an analysis and coding of the goals and objectives. Included is the total number of goals and objectives for each primary domain. Primary domains with corresponding sub-domains are summarized after the primary domains are presented. An 
analysis of the 10 sets of student data revealed 393 or $100 \%$ of goals and objectives being aligned with one of the domains and sub-domains overall. After the initial coding, all aligning goals and objectives represented all preschool primary domains: Approaches to Learning, Social and Emotional Development, Language and Communication, Literacy, Mathematics Development, Scientific Reasoning, and Perceptual, Motor, and Physical Development. There was less consistency within the sub domains of the framework, the presentation of data in the following tables will identify concentration and gaps in alignment. Table 4.3 provides a summary of the primary domains of the Head Start Early Leaner Outcome Framework (2015) and the current focus of instruction for children who are blind and use braille in preschool based on alignment of IFSP and IEP goals and objectives. Following this summary, each domain with corresponding sub domains will be analyzed and results presented. 
Table 4.1

Alignment of Individual Family Service Plans and Individual Education Plans with Head Start Early Learning Outcomes Framework: Primary Domains

\begin{tabular}{|c|c|c|c|c|c|c|c|c|c|c|}
\hline & $\stackrel{\bar{\sigma}}{0} \mathrm{z}$ & $\partial^{\circ}$ & 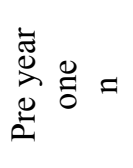 & $\partial^{0}$ & 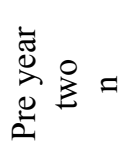 & $\partial^{\circ}$ & 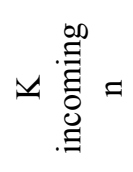 & $\partial^{0}$ & 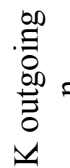 & $=0^{\circ}$ \\
\hline 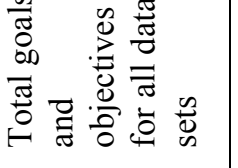 & $\hat{\text { nे }}$ & @̊̀ & ลे & iें & aे & $\begin{array}{l}\stackrel{0}{ } \\
\stackrel{n}{a}\end{array}$ & $\stackrel{2}{\varrho}$ & $\stackrel{\stackrel{\circ}{~}}{\lambda}$ & \& & ๗ें \\
\hline 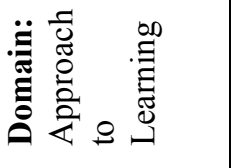 & $\bar{n}$ & $\stackrel{\circ}{\infty}$ & $a$ & $\stackrel{\text { ลे }}{\circ}$ & $\stackrel{0}{ }$ & $\stackrel{\stackrel{\circ}{n}}{\text { nे }}$ & $r$ & $\stackrel{\stackrel{0}{ }}{\grave{\lambda}}$ & $n$ & $\stackrel{d}{0}$ \\
\hline 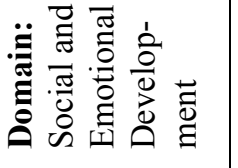 & ले & $\stackrel{\circ}{0}$ & $a$ & $\stackrel{\stackrel{े}{े}}{\text { ते }}$ & $\stackrel{0}{ }$ & ìं & $=$ & $\begin{array}{l}\stackrel{0}{\circ} \\
\text { ஸे }\end{array}$ & $a$ & $\stackrel{\circ}{\ddot{n}}$ \\
\hline 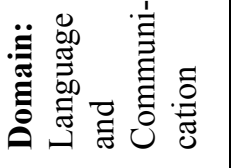 & $\dot{n}$ & $\stackrel{\stackrel{\circ}{ \pm}}{-}$ & $\infty$ & $\stackrel{\circ}{m}$ & $\stackrel{\infty}{-}$ & $\stackrel{\circ}{\grave{m}}$ & 6 & $\stackrel{\partial^{0}}{=}$ & 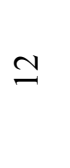 & ڤे \\
\hline 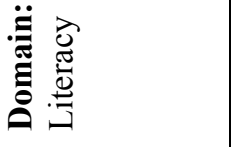 & $\infty$ & $\frac{\partial^{0}}{\vec{\nu}}$ & I & ठें & 9 & 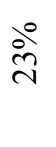 & లి & dें & $\stackrel{\sim}{ }$ & $\stackrel{\stackrel{\circ}{\sim}}{\sim}$ \\
\hline 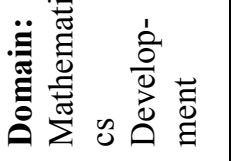 & $\stackrel{8}{0}$ & in & 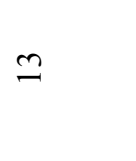 & ڤे̀ & 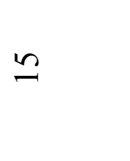 & iें & $\supseteq$ & ڤें & $\cong$ & ڤे \\
\hline 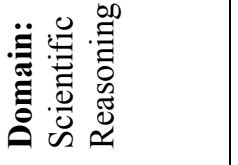 & $\infty$ & $\stackrel{\text { ते }}{ }$ & $N$ & 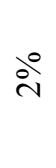 & - & $\stackrel{\text { ஸे }}{=}$ & - & $\stackrel{\text { ஸे }}{=}$ & $\nabla$ & ठें \\
\hline 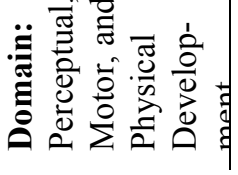 & $\stackrel{n}{=}$ & ڤे & $\bar{n}$ & $\stackrel{\stackrel{0}{\Delta}}{\grave{\lambda}}$ & $\stackrel{\sim}{\sim}$ & $\stackrel{\text { ते }}{\text { ते }}$ & $\bar{n}$ & $\stackrel{\Delta}{\stackrel{\Delta}{\circ}}$ & $\hat{\sim}$ & $\stackrel{\text { ஸे }}{\text { ஸे }}$ \\
\hline
\end{tabular}


Domain: Approaches to Learning. Table 4.2 is a summary of the primary domain Approaches to Learning with a summary of the corresponding sub domains.

Table 4.2

Primary Domain: Approaches to Learning with Sub Domains

\begin{tabular}{|c|c|c|c|c|c|c|c|c|c|c|}
\hline & $\begin{array}{c}\text { Total } \\
\mathrm{N}\end{array}$ & $\%$ & $\begin{array}{c}\text { Pre } \\
\text { year } \\
\text { one } \\
n\end{array}$ & $\%$ & $\begin{array}{c}\text { Pre } \\
\text { year } \\
\text { two } \\
n\end{array}$ & $\%$ & $\begin{array}{c}\mathrm{K} \\
\text { incoming } \\
\mathrm{n}\end{array}$ & $\%$ & $\begin{array}{c}\mathrm{K} \\
\text { outgoing } \\
\mathrm{n}\end{array}$ & $\%$ \\
\hline $\begin{array}{l}\text { Domain: } \\
\text { Approaches } \\
\text { to learning }\end{array}$ & 31 & $8 \%$ & 9 & $29 \%$ & 10 & $32 \%$ & 7 & $23 \%$ & 5 & $16 \%$ \\
\hline $\begin{array}{l}\text { Emotional } \\
\text { self } \\
\text { regulation }\end{array}$ & 29 & $94 \%$ & 8 & $27 \%$ & 9 & $31 \%$ & 7 & $24 \%$ & 5 & $17 \%$ \\
\hline $\begin{array}{l}\text { Cognitive } \\
\text { self- } \\
\text { regulation } \\
\text { (executive } \\
\text { functioning) }\end{array}$ & 0 & $0 \%$ & 0 & $0 \%$ & 0 & $0 \%$ & 0 & $0 \%$ & 0 & $0 \%$ \\
\hline $\begin{array}{l}\text { Initiative } \\
\text { and } \\
\text { curiosity }\end{array}$ & 0 & $0 \%$ & 0 & $0 \%$ & 0 & $0 \%$ & 0 & $0 \%$ & 0 & $0 \%$ \\
\hline Creativity & 2 & $6 \%$ & 1 & $50 \%$ & 1 & $50 \%$ & 0 & $0 \%$ & 0 & $0 \%$ \\
\hline
\end{tabular}

An analysis of goals and objectives in the primary domain of Approaches to Learning $(n=31 ; 8 \%)$ indicated a predominate focus on the sub domain of Emotion and Behavioral Self Regulation ( $n=29 ; 94 \%)$ with a small number aligned with sub domain of Creativity $(n=2 ; 6 \%)$ and no goals or objectives aligned with the sub domain of Cognitive Self-Regulation (Executive Functioning) or Initiative and Curiosity. The goals aligned with Emotional and Behavioral Self Regulation concerned transition and participation in classroom routines, as well as arrival and departure routines. In addition, 
goals and objectives were identified for following directions and maintaining focus on activities. Two students had goals and objectives focusing on identifying and problem solving behavior that included vocal abuse, agitation and anxiety. The goal and objective in the sub-domain of Creativity was to explore and play with pretend materials, this goal was for the same student, two consecutive years.

Primary domain: Social and Emotional Development. Table 4.3 is a summary of the primary domain Social and Emotional Development with a summary of the corresponding sub domains.

Table 4.3

Primary Domain: Social and Emotional Development with Sub Domains

\begin{tabular}{|c|c|c|c|c|c|c|c|c|c|c|}
\hline & $\begin{array}{c}\text { Total } \\
\mathrm{N}\end{array}$ & $\%$ & $\begin{array}{c}\text { Pre } \\
\text { year } \\
\text { one } \\
n\end{array}$ & $\%$ & $\begin{array}{c}\text { Pre } \\
\text { year } \\
\text { two } \\
n\end{array}$ & $\%$ & $\begin{array}{c}\mathrm{K} \\
\text { incoming } \\
\mathrm{n}\end{array}$ & $\%$ & $\begin{array}{c}\mathrm{K} \\
\text { outgoing } \\
\mathrm{n}\end{array}$ & $\%$ \\
\hline $\begin{array}{l}\text { Domain: } \\
\text { Social and } \\
\text { emotional } \\
\text { development }\end{array}$ & 39 & $10 \%$ & 9 & $23 \%$ & 10 & $26 \%$ & 11 & $28 \%$ & 9 & $23 \%$ \\
\hline $\begin{array}{l}\text { Relationship } \\
\text { with adults }\end{array}$ & 3 & $7 \%$ & 0 & $0 \%$ & 0 & $0 \%$ & 2 & $67 \%$ & 1 & $33 \%$ \\
\hline $\begin{array}{l}\text { Relationship } \\
\text { with other } \\
\text { children }\end{array}$ & 34 & $87 \%$ & 9 & $26 \%$ & 9 & $26 \%$ & 8 & $23 \%$ & 8 & $23 \%$ \\
\hline $\begin{array}{l}\text { Emotional } \\
\text { functioning }\end{array}$ & 2 & $5 \%$ & 0 & $0 \%$ & 1 & $50 \%$ & 1 & $50 \%$ & 0 & $0 \%$ \\
\hline $\begin{array}{l}\text { Sense of } \\
\text { identity and } \\
\text { belonging }\end{array}$ & 0 & $0 \%$ & 0 & $0 \%$ & 0 & $0 \%$ & 0 & $0 \%$ & 0 & $0 \%$ \\
\hline
\end{tabular}


An analysis of goals and objectives in the primary domain of Social and

Emotional Development $(n=30 ; 10 \%)$ indicated goals aligned with three of the four subdomains. The majority of goals and objectives were aligned with the sub-domain of Relationship with Other Children $(n=34 ; 87 \%)$ minimal alignment with Relationship with Adults $(n=3 ; 7 \%)$ and Emotional Functioning $(n=2 ; 5 \%)$; there were no goals or objectives aligned with the sub-domain of Sense of Identity and Belonging. Goals and objectives aligned with Relationship with Other Children focused on responding to peer comments and questions, initiating conversation, playing collaboratively, and initiating play with peers. Relationship with Adults included goals and objectives regarding turn taking with adults and completing adult requests. The goal in the area of Emotional Functioning was to label emotions, tell an adult how the student was feeling and to use self calming techniques. This goal was written for two consecutive years for the same student.

Primary domain: Language and Communication. Table 4.4 is a summary of the primary domain Language and Communication with a summary of the corresponding sub domains. 
Table 4.4

Primary Domain: Language and Communication with Sub Domains

\begin{tabular}{|c|c|c|c|c|c|c|c|c|c|c|}
\hline & $\begin{array}{c}\text { Total } \\
\mathrm{N}\end{array}$ & $\%$ & $\begin{array}{c}\text { Pre } \\
\text { year } \\
\text { one } \\
n\end{array}$ & $\%$ & $\begin{array}{l}\text { Pre } \\
\text { year } \\
\text { two } \\
n\end{array}$ & $\%$ & $\begin{array}{c}\mathrm{K} \\
\text { incoming } \\
\mathrm{n}\end{array}$ & $\%$ & $\begin{array}{c}\mathrm{K} \\
\text { outgoing } \\
\mathrm{n}\end{array}$ & $\%$ \\
\hline $\begin{array}{l}\text { Domain: } \\
\text { Language and } \\
\text { communication }\end{array}$ & 54 & $14 \%$ & 18 & $33 \%$ & 18 & $33 \%$ & 6 & $11 \%$ & 12 & $22 \%$ \\
\hline $\begin{array}{l}\text { Attending and } \\
\text { understanding }\end{array}$ & 12 & $22 \%$ & 4 & $33 \%$ & 5 & $41 \%$ & 1 & $8 \%$ & 2 & $17 \%$ \\
\hline $\begin{array}{l}\text { Communicating } \\
\text { and speaking }\end{array}$ & 24 & $44 \%$ & 6 & $25 \%$ & 6 & $25 \%$ & 4 & $17 \%$ & 8 & $33 \%$ \\
\hline Vocabulary & 18 & $33 \%$ & 8 & $44 \%$ & 7 & $39 \%$ & 1 & $5 \%$ & 2 & $11 \%$ \\
\hline
\end{tabular}

The domain of Language and Communication $(n=54 ; 14 \%)$ was the most evenly aligned domain with alignment in all three sub-domains, Attending and Understanding ( $n$ $=12 ; 22 \%)$, Communicating and Speaking $(n=24 ; 44 \%)$ and Vocabulary $(n=18 ; 33 \%)$. Attending and Understanding was focused on responding to verbal requests particularly in the area of orientation and mobility concepts. Concepts included prepositions, directional words, stop and listen. Other goals and objectives included answering who, what, where, yes, no questions, greeting and recognizing peers, following directions containing prepositions and locating specific locations within their school as directed. Communicating and Speaking included communicating effectively in a variety of situations, raising hand to solicit attention, request, describe or protest. The sub domain of Vocabulary included naming objects when function is described, naming objects by 
touch, using words to describing textures and objects, understanding orientation and mobility concepts including positional concepts.

Primary domain: Literacy. Table 4.5 is a summary of the primary domain of Literacy with a summary of the corresponding sub domains.

Table 4.5

Primary Domain: Literacy with Sub Domains

\begin{tabular}{|c|c|c|c|c|c|c|c|c|}
\hline $\begin{array}{c}\text { Total } \\
\mathrm{N}\end{array}$ & $\%$ & $\begin{array}{c}\text { Pre } \\
\text { year } \\
\text { one } \\
n\end{array}$ & $\%$ & $\begin{array}{c}\text { Pre } \\
\text { year } \\
\text { two } \\
n\end{array}$ & $\%$ & $\underset{n}{\mathrm{~K}}$ & $\%$ & $\begin{array}{c}\mathrm{K} \\
\text { outgoing } \\
\mathrm{n}\end{array}$ \\
\hline
\end{tabular}

\begin{tabular}{|c|c|c|c|c|c|c|c|c|c|c|}
\hline $\begin{array}{l}\text { Domain: } \\
\text { Literacy }\end{array}$ & 86 & $21 \%$ & 17 & $20 \%$ & 19 & $23 \%$ & 30 & $36 \%$ & 20 & $24 \%$ \\
\hline $\begin{array}{l}\text { Phonological } \\
\text { awareness }\end{array}$ & 1 & $1 \%$ & 0 & $0 \%$ & 0 & $0 \%$ & 0 & $0 \%$ & 1 & 100 \\
\hline $\begin{array}{l}\text { Print and } \\
\text { alphabet } \\
\text { knowledge }\end{array}$ & 55 & $64 \%$ & 12 & $22 \%$ & 12 & $22 \%$ & 18 & $33 \%$ & 13 & $24 \%$ \\
\hline $\begin{array}{l}\text { Comprehensive } \\
\text { text structure }\end{array}$ & 0 & $0 \%$ & 0 & $0 \%$ & 0 & $0 \%$ & 0 & $0 \%$ & 0 & $0 \%$ \\
\hline Writing & 30 & $35 \%$ & 5 & $17 \%$ & 7 & $23 \%$ & 12 & $40 \%$ & 6 & $20 \%$ \\
\hline
\end{tabular}

The Literacy domain $(n=86 ; 21 \%)$ was notable in the vast majority of goals and objectives aligned with Print and Alphabetic knowledge $(n=55 ; 64 \%)$ and Writing $(n=$ $30 ; 35 \%)$. One goal was aligned with Phonological Awareness $(n=1 ; 1 \%)$. There was no alignment between the goals and objectives and the sub domains of Comprehension and Text Structure. Goals and objectives in Print and Alphabetic Knowledge during preschool were focused on correct finger placement and tracking, understanding spatial position of braille dots, exploring the braille page, and orientation of the page and 
identifying braille activities. Goals and objectives also included locating their name in braille and recognizing letters in their first name. In kindergarten, the goals in Alphabet and Print Knowledge included knowing all letters of the alphabet, upper and lower case, whole and part word contractions, alphabet contractions, literary numbers, and punctuation signs including capital and number sign. One student had a goal which included reading consonant-vowel-consonant words in braille, and one student had a goal of increasing reading in the area of phonetic and alphabet sounds; these were the goals which referenced phonetics. The sub domain of Writing $(n=30 ; 35 \%)$ followed a similar pattern to Alphabet and Print Knowledge. In preschool, the focus was on correct hand placement, learning parts of the braille writer, functions of a braille writer and brailling letters in their name. In kindergarten, goals and objectives included forming the braille cell on a peg board, writing with a braille writer, naming parts of the braille writer, loading and removing braille paper, back space, and finger placement. There were also goals and objectives which included writing literary numbers, upper and lower case alphabet letters, as well as the capital and number sign.

Primary domain: Mathematics Development. Table 4.6 is a summary of the primary domain of Mathematics Development with a summary of the corresponding sub domains. 
SCHOOL READINESS: CHILDREN WHO ARE BLIND

Table 4.6

Primary Domain: Mathematics Development with Sub Domains

\begin{tabular}{|c|c|c|c|c|c|c|c|c|c|c|}
\hline & $\begin{array}{c}\text { Total } \\
\mathrm{N}\end{array}$ & $\%$ & $\begin{array}{c}\text { Pre } \\
\text { year } \\
\text { one } \\
n\end{array}$ & $\%$ & $\begin{array}{c}\text { Pre } \\
\text { year } \\
\text { two } \\
n\end{array}$ & $\%$ & $\begin{array}{c}\mathrm{K} \\
\text { incoming } \\
\mathrm{n}\end{array}$ & $\%$ & $\begin{array}{c}\mathrm{K} \\
\text { outgoing } \\
\mathrm{n}\end{array}$ & $\%$ \\
\hline $\begin{array}{l}\text { Domain: } \\
\text { Mathematics } \\
\text { development }\end{array}$ & 60 & $15 \%$ & 11 & $22 \%$ & 13 & $25 \%$ & 18 & $32 \%$ & 18 & $22 \%$ \\
\hline $\begin{array}{l}\text { Counting and } \\
\text { cardinality }\end{array}$ & 38 & $63 \%$ & 4 & $14 \%$ & 6 & $21 \%$ & 13 & $45 \%$ & 15 & $39 \%$ \\
\hline $\begin{array}{l}\text { Operations and } \\
\text { algebraic } \\
\text { thinking }\end{array}$ & 7 & $14 \%$ & 1 & $14 \%$ & 1 & $14 \%$ & 3 & $43 \%$ & 2 & $29 \%$ \\
\hline Measurement & 1 & $2 \%$ & 1 & $100 \%$ & 0 & $0 \%$ & 0 & $0 \%$ & 0 & $0 \%$ \\
\hline $\begin{array}{l}\text { Geometry and } \\
\text { spatial sense }\end{array}$ & 14 & $27 \%$ & 5 & $36 \%$ & 6 & $43 \%$ & 2 & $14 \%$ & 1 & $7 \%$ \\
\hline
\end{tabular}

In the domain of Mathematics Development $(n=60 ; 15 \%)$ alignment was predominately with the sub-domains of Counting and Cardinality $(n=38 ; 63 \%)$ and Geometry and Spatial Sense $(n=14 ; 27 \%)$. The sub-domains of Operations and Algebraic Thinking $(n=7 ; 14 \%)$ and Measurement $(n=1 ; 2 \%)$ were also represented. In preschool Counting and Cardinality goals and objectives were focused primarily on counting, one to one correspondence, quantitative concepts such as many, few, one, two, and using the abacus to count up to 20 . In kindergarten, goals in this sub domain continued to focus on pre-academic goals including matching, sorting and counting. Goals and objectives also focused on learning nemeth numbers to 100 , nemeth math 
symbols, and simple computation up to 5 . Nemeth is a separate braille code specifically for numbers and mathematical computation. In the sub domain of Operation and Algebraic Thinking, goals and objectives included matching simple sequences or patterns, showing and completing $\mathrm{ABAB}$ and $\mathrm{AABB}$ patterns as well as using the abacus to add and subtract one digit numbers. In the sub domain of Measurement, ordering objects horizontally by size was the single goal and objective listed.

Primary domain: Scientific Reasoning. Table 4.7 is a summary of the primary domain of Scientific Reasoning with a summary of the corresponding sub domains. Table 4.7

Primary Domain: Scientific Reasoning with Sub Domains

\begin{tabular}{|c|c|c|c|c|c|c|c|c|c|c|}
\hline & $\begin{array}{c}\text { Total } \\
\mathrm{N}\end{array}$ & $\%$ & $\begin{array}{c}\text { Pre } \\
\text { year } \\
\text { one } \\
n\end{array}$ & $\%$ & $\begin{array}{c}\text { Pre } \\
\text { year } \\
\text { two } \\
n\end{array}$ & $\%$ & $\begin{array}{c}\mathrm{K} \\
\text { incoming } \\
\mathrm{n}\end{array}$ & $\%$ & $\begin{array}{c}\mathrm{K} \\
\text { outgoing } \\
\mathrm{n}\end{array}$ & $\%$ \\
\hline $\begin{array}{l}\text { Domain: } \\
\text { Scientific } \\
\text { Reasoning }\end{array}$ & 8 & $2 \%$ & 2 & $2 \%$ & 1 & $13 \%$ & 1 & $13 \%$ & 4 & $50 \%$ \\
\hline $\begin{array}{l}\text { Scientific } \\
\text { Inquiry }\end{array}$ & 8 & $100 \%$ & 2 & $25 \%$ & 1 & $25 \%$ & 1 & $2 \%$ & 4 & $50 \%$ \\
\hline $\begin{array}{l}\text { Scientific } \\
\text { Reasoning \& } \\
\text { problem } \\
\text { solving }\end{array}$ & 0 & $0 \%$ & 0 & $0 \%$ & 0 & $0 \%$ & 0 & $0 \%$ & 0 & $0 \%$ \\
\hline
\end{tabular}

The domain of Scientific Reasoning $(n=8 ; 2 \%)$ was aligned in the sub-domain of Scientific Inquiry $(n=8 ; 100 \%)$; there were no goals or objectives aligned with Scientific Reasoning and Problem Solving. This sub domain included goals and objectives requiring children to sort and group objects based on visual, tactual, color, shape or size characteristics. 
Primary domain: Perceptual, Motor, and Physical Development. Table 4.8 is a summary of the primary domain of Perceptual, Motor, and Physical Development with a summary of the corresponding sub domains.

Table 4.8

Primary Domain: Perceptual, Motor, and Physical Development with Sub Domains.

\begin{tabular}{|c|c|c|c|c|c|c|c|c|c|c|}
\hline & $\begin{array}{c}\text { Total } \\
\mathrm{N}\end{array}$ & $\%$ & $\begin{array}{c}\text { Pre } \\
\text { year } \\
\text { one } \\
n\end{array}$ & $\%$ & $\begin{array}{c}\text { Pre } \\
\text { year } \\
\text { two } \\
n\end{array}$ & $\%$ & $\begin{array}{c}\mathrm{K} \\
\text { incoming } \\
\mathrm{n}\end{array}$ & $\%$ & $\begin{array}{c}\mathrm{K} \\
\text { outgoing } \\
\mathrm{n}\end{array}$ & $\%$ \\
\hline $\begin{array}{l}\text { Domain: } \\
\text { Perceptual, } \\
\text { motor, and } \\
\text { physical } \\
\text { development }\end{array}$ & 115 & $29 \%$ & 31 & $27 \%$ & 26 & $23 \%$ & 31 & $27 \%$ & 27 & $23 \%$ \\
\hline Gross motor & 62 & $54 \%$ & 16 & $26 \%$ & 11 & $18 \%$ & 19 & $31 \%$ & 16 & $26 \%$ \\
\hline Fine motor & 30 & $26 \%$ & 9 & $30 \%$ & 9 & $30 \%$ & 6 & $20 \%$ & 6 & $20 \%$ \\
\hline $\begin{array}{l}\text { Health, safety } \\
\text { and nutrition }\end{array}$ & 23 & $20 \%$ & 6 & $26 \%$ & 6 & $26 \%$ & 6 & $26 \%$ & 5 & $22 \%$ \\
\hline
\end{tabular}

The domain of Perceptual, Motor, and Physical development ( $n=115 ; 29 \%)$ was the most represented when reviewing IFSP and IEP documents. The sub-domain of Gross Motor $(n=62 ; 54 \%)$, Fine Motor $(n=30 ; 26 \%)$ and Health, Safety, and Nutrition $(n=23 ; 20 \%)$ were consistently listed in data sets for students who are blind and use braille as their primary medium. Gross motor skills were consistently written within the area of orientation and mobility including cane skills such as constant contact, diagonal technique, detecting drop offs, landmarks and environmental clues, sighted guide and 
learning routes within the school building, play ground area and residential neighborhood. Fine motor skills included two-handed activities to improve finger and hand strength, use of scissors, lacing, manipulating materials and two-hand coordination. Health, safety, and nutrition was represented by goals in the area of clothing manipulation, independence in toileting and self feeding as well as goals and objectives in following safety commands when traveling.

When reflecting back on journaling and memo writing two themes were identified; the first theme was the considerable change in education services provided in preschool versus kindergarten. The data indicated a large increase in time provide in kindergarten versus preschool, however, there was not a large increase in the number of goals and objectives. The second theme identified was the inconsistency of goals and objectives including clarity, continuity and quality. It was noted that many of the goals were not written in clearly measurable terms, there was occurrence of the same goal repeated over years, as well as non-sequential goals and multiple objectives within one goal.

\section{Expanded core curriculum}

Table 4.9 presents the data that indicated the areas of the expanded core curriculum focused on during preschool and kindergarten years. 
SCHOOL READINESS: CHILDREN WHO ARE BLIND

113

Table 4.9

Analysis of Expanded Core Curriculum

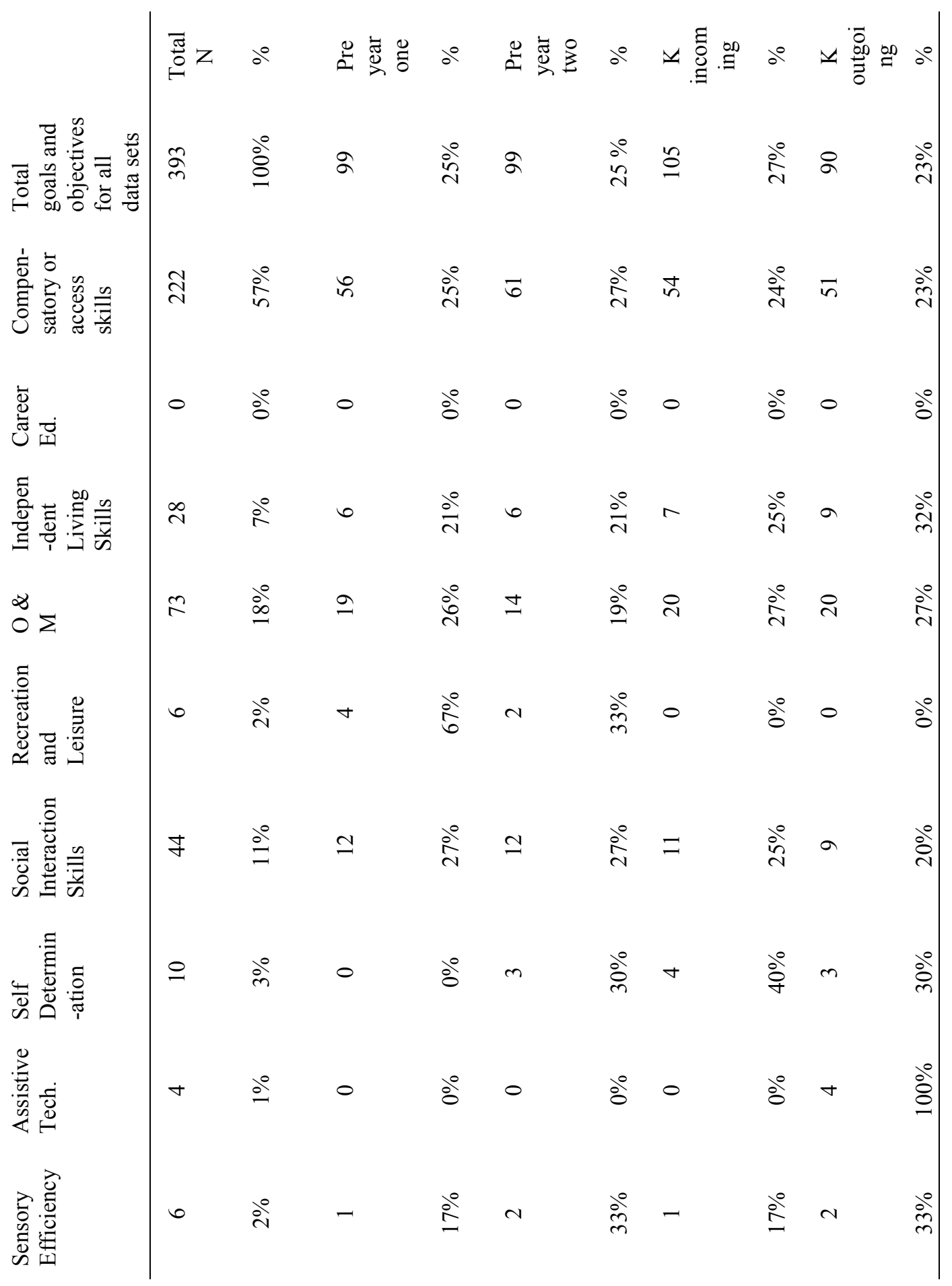


The majority of goals and objectives $(n=222 ; 57 \%)$ fell within the ECC area compensatory or academic skills including modes of communication. The next largest categories of the ECC were orientation and mobility $(n=73 ; 18 \%)$ and social interaction skills $(n=44 ; 11 \%)$. The remaining areas included independent living skills $(n=28$; $7 \%)$, self determination $(n=10 ; 3 \%)$, recreation and leisure $(n=6 ; 2 \%)$, sensory efficiency $(n=6 ; 2 \%)$, and assistive technology $(n=4 ; 1 \%)$. There were no goals or objectives listed that were coded in the area of career education.

Overall the focus of the ECC was focused in the area of braille readiness. As cited above, the focus was predominately on tactile readiness and the mechanics of the braille code. The other major area of the ECC was orientation and mobility, including both travel skills as well as environmental concept development. Goals and objectives were identified for independence in toileting and self-feeding which would align with the ECC area of independent living skills. Self-determination was reflected in one goal and objective for a student to raise their hand to solicit help as well as communicate emotional/physical needs. One goal was listed in the area of assistive technology that included learning to use digital books. Sensory efficiency was limited as students were identified braille users, however, there were two goals listed for increased visual discrimination.

\section{Education service time}

All 10 data sets reflected children attending preschool placements at age three and four; eight students attended specialized preschool placements (i.e. through early 
childhood special education [ECSE]) for two days per week, one student attended Head Start for four days per week and one student attended community preschool for two days per week. In addition to services from a TVI all students received direct instruction from special education professionals through ECSE or school age special education personnel. Students also received a variety of related services through speech language pathologists, occupational, and physical therapists and orientation and mobility specialists (see Table 3.1).

Figure 4.1 shows IFSP and IEP service time averages from TVIs during early childhood special education (preschool) and school age services entering and exiting kindergarten. Overall, there was an increase in 300 minutes per week from the TVI between the last year of preschool (68.3) and the kindergarten school year (366.7).

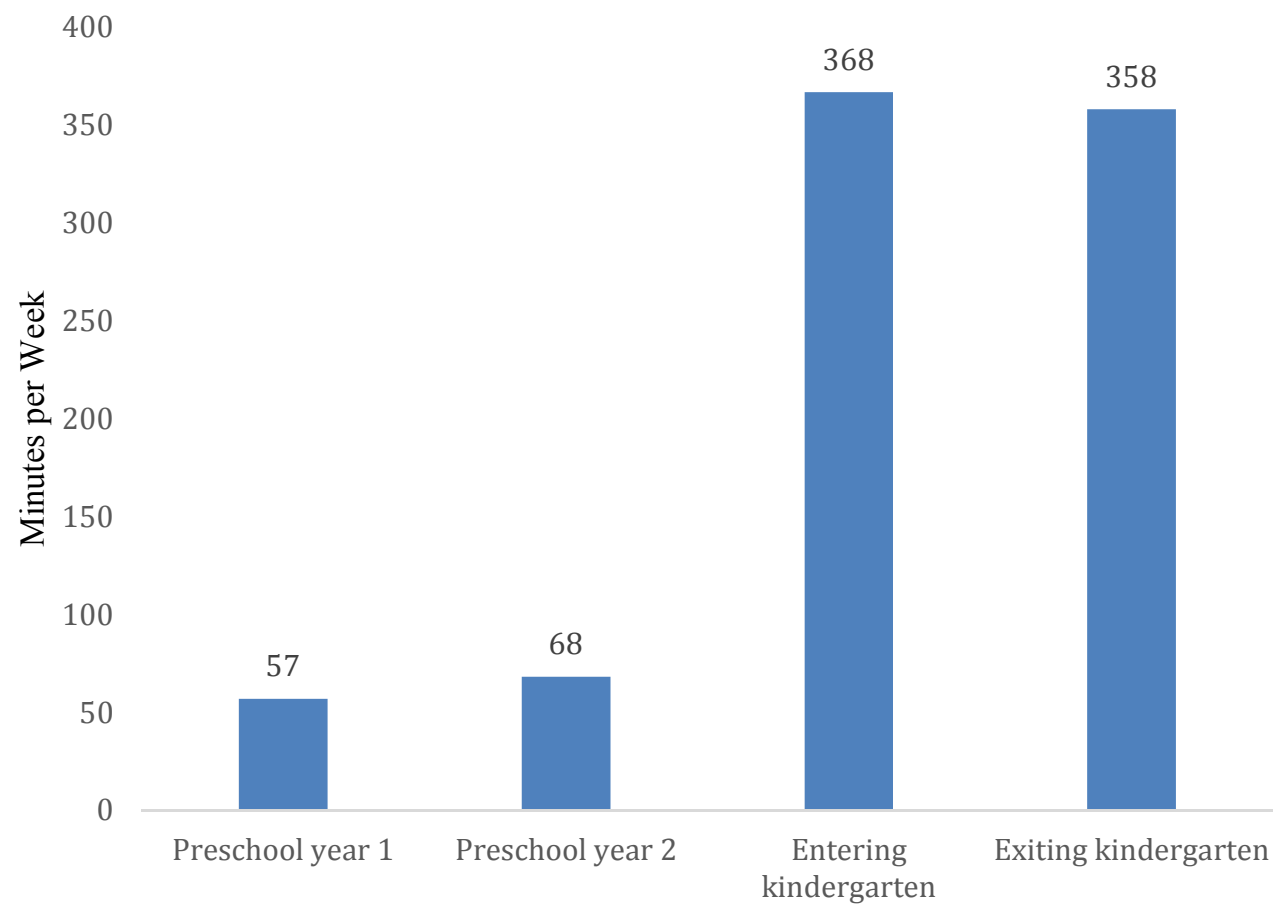

Figure 4.1. Teacher of the visually impaired Service: Minutes per week. 
Figure 4.2 shows minutes per week provided by an orientation and mobility specialist. As with the TVI, there was an increase in average time provided as the entered school age services from an average of 17 minutes per week in preschool to an average of 27 minutes per week in kindergarten.

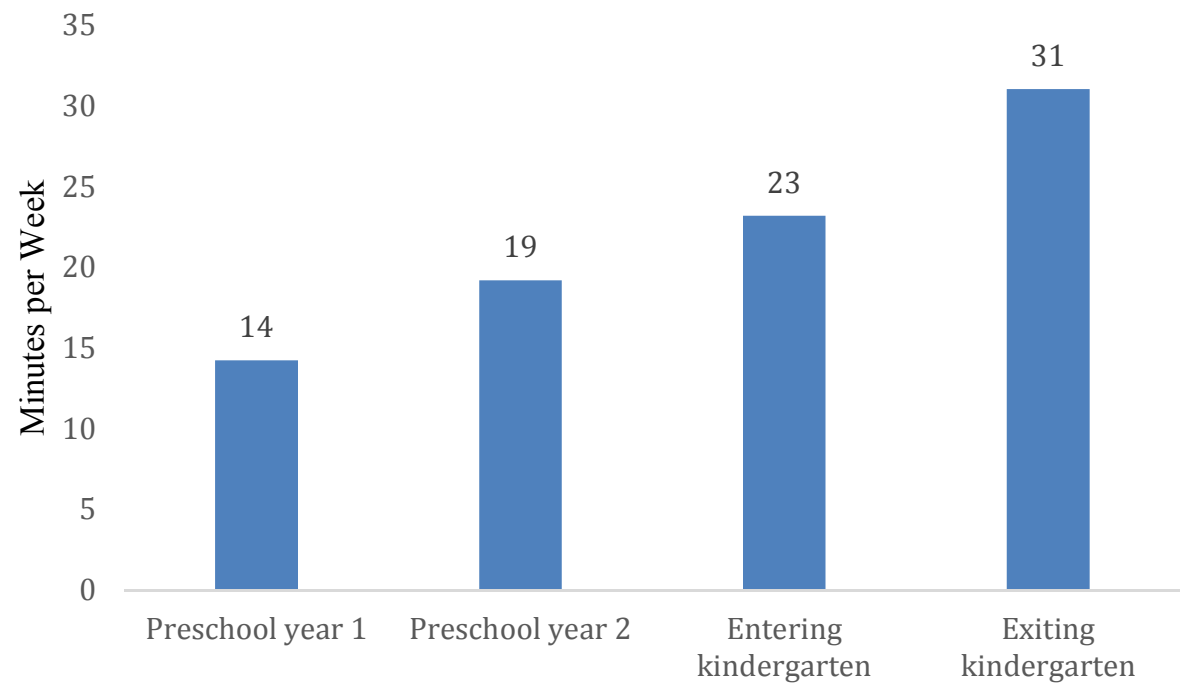

Figure 4.2. Orientation and mobility service: Minutes per week.

\section{Interview with teacher of the visually impaired}

Five TVIs participated in semi-structured interviews. Participants were from three regions in Oregon. All had taught for more than 10 years and had provided early intervention, early childhood special education as well as school age services. Four of the five participants interviewed were also certified in orientation and mobility. Three interviews were completed face to face, while two were completed over the phone. After interviews were complete and transcribed, I used a line-by-line coding process for initial coding. Next, a focused selective process of coding was used that identified, organized and analyzed data based on the listed research questions and organize large data from interviews (Charmaz, 2014). 
Semi-structured interviews with TVIs were intended to answer the primary research question: How is the construct of school readiness defined for children who are blind and use braille as their primary medium? Additional research questions included: (a) What is the current focus of instruction for children who are blind in preschool to prepare them for kindergarten? (b) What is the role of the TVI and primary caregiver in preparing students for kindergarten? (c) What do TVIs and primary caregivers identify as the barriers to school readiness for children who are blind? (d) What do TVIs and primary caregivers see as essential to preparing children who are blind for kindergarten? As the interviews were semi-structured, the answers to these questions emerged through coding, memo writing, journaling and reflection.

\section{How is the construct of school readiness defined for children who are blind} and use braille as their primary medium? All five participants noted braille readiness as a component of school readiness. Skills noted were correct finger placement, phonemic awareness, braille exposure, tactile books, left to right reading and tracking, understanding the braille cell, knowing most of their alphabet and their name in braille.

One interviewee captured the sense of educating the whole child "It's not just braille, I think it's the whole kindergarten experience.” Four out of the five TVIs also emphasized social emotional skills and approaches to learning. This was displayed in a quote from one interviewee who noted "I would say for me really important is social skills, being able to ask for help, being able to interact with peers, being able to wait their turn, able to transition, knowing terms, lining up." Another TVI expressed social skills as including "being able to ask for help, being able to interact with peers, being able to wait 
their turn, being able to transition from circle time to gym, and knowing the terms such as lining up."

Academic and behavioral expectations were a key element of kindergarten readiness. When asked how kindergarten readiness is defined for a child who is blind, the TVI said "The expectations should be the same that they are ready at that point or have some of the basic knowledge to be ready to be successful in school. Some of the soft skills too like being able to sit and listening to directions and following directions." Two out of the five TVIs reflected on increased academic expectations in preschool, "I think anymore with the way the Common Core and Kindergarten Readiness, they've really moved. In my opinion, second grade is what first grade is doing now, Kindergarten is doing first grade work, preschool should be doing kindergarten work, and they're just not ready for it."

\section{What is the current focus of instruction for children who are blind in} preschool to prepare them for kindergarten? All of the TVIs interviewed discussed pre-braille and exposure to braille as a focus of instruction for children who are blind in preschool. Every TVI discussed the need for tactile development and foundational skills of the braille code. "There is a whole set of tactile skills, the tactile skills of being able to track and all of those dynamics but also knowing what a book is, knowing top to bottom, having lots of reading experiences." Introduction of the braille writer and braille writing was discussed in interviews including understanding the braille cell, concepts of braille characters formed, "How the story comes together through the braille characters." 
A noted theme identified was TVIs identifying a focus in providing the child access to braille in multiple environments. "It depends a lot on the environment. And I think my biggest job is to work with that teacher and the assistants in the class to get them really interested in braille." One TVI noted, "It's harder because they are not immersed into braille, kids see print every day, even if its on cereal boxes, kids who need braille can't see the print and don't get as much exposure so they need a lot more exposure than even kids who use print because there is little incidental learning when it comes to the literacy skills." Two out of the five TVIs mentioned using the Oregon Project Skills Inventory (Brown, et al., 1978) as a tool to identify goals and objectives for preschool children who were blind. Two TVIs mentioned the Assessment, Evaluation, and Programming System for Infants and Children (AEPS) (Bricker \& Squires, 1999).

What is the role of the TVI and primary caregiver in preparing students for kindergarten? When this question was posed, all TVIs mentioned the importance of family involvement in preparing children who are blind for kindergarten. A theme repeated was the difficulty in teaching braille to families, their willingness and resources to learn the braille code. "It's my goal to help parents learn braille, it's really about parent training, so they feel comfortable, they understand it's not super complicated and that it's not scary." Another TVI stated "The parents are a huge part of readiness especially if we got the parents into doing braille and having the student immersed in the braille environment." Also noted were barriers to teaching parents or caregivers braille such as materials, time, resources, and willingness of to learn. "I see a lot of fear and 
uncertainty and that of course can be one of those barriers, if the family is balking at braille, the time it might take, so having realistic expectations and guiding them along."

This question led to reflections of the parent grieving process and the role of the TVI in supporting families in learning and understanding braille. "I've had parents be really upset that their child is going to have to learn braille. I've had parents say they want no braille in their house." Another TVI notes, "it's even harder for our kids when we have limited time and resources and parents go through the grieving process so it's hard because you are dealing with that too."

\section{What do TVIs and identify as the barriers to school readiness for children} who are blind? What do TVIs see as essential to preparing children who are blind for kindergarten? These questions elicited the most dialogue from the TVIs. The consensus of participants was there was not enough time to teach skills in kindergarten readiness and the expanded core curriculum. One TVI reflected the comments by stating I would see them at preschool and I would see them at home as well, four hours a day, that would be fabulous, and mobility and all that other stuff another two hours! All day would be beneficial! There is so much for a child who is blind to learn that sighted students learn by just watching the world. Sometimes it's hard to know even where to start.

Participants expressed frustration regarding competing demands on their time including paperwork, assessment, eligibility, meetings, materials preparation, coaching, and training district staff and direct instruction to the child. "Our kids need so much more than a lot of other kids, they need a lot more instruction and a lot of it is staff and family 
training and engagement." There is also the concern that general education staff does not understand the time and extent of material adaptation required. "I think another barrier is just the staff not realizing how much materials need to be adapted or what's really important to adapt."

The structure of time for TVIs was also a concern, "I think going every day would be wonderful if there were no service constraints. I think that maybe short visits. And if there were not constraints, then I could go different times of the day.” The same TVI continued to discuss the difficulty of scheduling in early childhood special education given the time students are in preschool, the nature of home visiting and short attention span of four and five year children. Finally, one TVI noted the structure of caseloads as well as specially designed instruction determined on individual education plans. "In many ways we need to invert our services, to really hit that young, and get in there with the family because it's time consuming." When I verified with the participant, he was referring to services specifically for secondary students from TVIs that could be reduced as the student gains skills and moves toward independence.

TVI participants echoed research noting children who are blind demonstrate developmental lags. "I guess what I see now is they are pushing kids to read earlier and earlier. And developmentally, I don't think they're quite they're quite there. And our kids who are blind are developmentally a little bit delayed anyway." A theme that emerged was the need for students using braille to be immersed in braille to have increased opportunities for incidental learning similar to their sighted peers. "I think in the best possible world there would be a preschool with braille in it and that would be 
around them all of the time and direct instruction from the TVI. Definitely a lot more than we are doing now if we had no constraints in time." All five TVIs interviewed agreed more time in early childhood special education services would improve outcomes for children who are blind and better prepare them for kindergarten.

\section{Interview with Primary Caregivers}

Three primary caregivers were interviewed; their children are currently reading braille as their primary learning medium. Four themes emerged from the data: (a) feeling of grief and isolation, (b) exposure to the braille code in preschool, (c) entering kindergarten significantly behind and, (d) the importance of communication between the family and educators (both TVIs and general education teachers). In addition, it is important to note that for two of the three children the determination of learning media was both print and braille in preschool. In hindsight, primary caregivers see this as a cause of further delay and confusion with the braille code.

Without exception, primary caregivers talked about grief and isolation they felt having a child who was blind. "In the early years, as a parent, I really didn't know what was going on, I didn't know any blind kids or any friends who had disabled children and these were my first two kids, you are kind of thrown into the situation." As a semistructured interview, the participants in part guided the conversation. Two of the participants talked about frustration with medical providers and understanding the extent of their child's disability. "I remember when he was two and I took him for his two-year evaluation and they said he had neurological deficits and I shouldn't get my hopes up. I was completely devastated." Another primary caregiver relayed, "I thought she had a 
lazy eye and we went to the doctor. That's when the doctor came back and said she's blind, she'll never be able to see. Which was a huge shock, and there were no resources from the doctor." She went on to explain she had not familiarity with individuals with vision impairments, did not understand there were different degrees of vision loss and received no information regarding educational services. All of the primary caregivers interviewed talked about grief and isolation with their children experiencing a low incidence disability; all three also talked about gratitude for regional program services from teachers of the visually impaired. "I can't tell you the amount of help and resourcesI've received from you guys, unless you can get to you first you are just stuck in this ‘what do I do now?' phase.”

Primary caregivers confirmed the predominant focus of instruction in preschool for their children was exposure to braille. This included an introduction to the braille code but no expectation their children enter kindergarten reading. "There was no expectation for [child's name] to learn braille" another parent reported, "They were working on her name in writing, but not using braille". A third parent stated that "In preschool it was mostly textures and meeting new kids was all we really focused on". One caregiver relayed "I remember asking and they said, 'Oh he won't learn braille until he goes to kindergarten, that's where they teach the braille'."

Two primary caregivers discussed their children being tactually defensive and needing exposure and direct instruction to tactile skills and access to the code. "She didn't like to touch anything; she was very hesitant about touching things." Additionally, all three primary caregivers talked about their children learning the letters in their name, 
the names of the letter such as the alphabet song. "Looking back on it now, I wish I would have, I should have said, you know, [child's name] should be doing what every other kid is doing." One primary caregiver summarized "I think preschool was focusing on personal growth and then when we got to kindergarten we were trying to do the academic part and it was a huge struggle. I don't think she was as prepared for kindergarten as she should have been."

Each primary caregiver discussed how their child entered kindergarten already behind their sighted peers. One caregiver talked about how she asked for a bridge year because she didn't feel her child was ready to go to kindergarten. The consequence of another year of preschool would have been a year without services as the child would have been too old for Early Childhood Special Education and not enrolled in a school district. Primary caregivers discussed the struggle of their children learning the alphabet, taking the whole year to learn the alphabet code. "When they get older there's not the time to learn the alphabet and contractions and stuff so if you don't start from the very beginning it halts progress a lot, trying to learn in the middle of learning everything else." One caregiver discussed the consequence of when direct instruction in braille occurred in the hallway, removing her child from the general education classroom. Her child was removed due in part to the general education teacher believing the braille writer was too loud and disruptive in the classroom. To her, this was indicative of the advocacy that was required by her as a parent for her child to attend a general education classroom. It should be noted that all three primary caregivers' children were attending their neighborhood school in general education classes. 
Primary caregivers discussed their experiences with communication with TVIs as well as communication with the general education staff and school districts. Participants discussed the importance of regular communication with the TVI regarding their child's progress as well as the provision of resources to support instruction at home. "To me this [communication] is one of the most important things that I struggle with on a daily basis." Primary caregivers expressed it was critical for them to understand progress and what was happening at school on a regular basis. They also talked about the difficulties in explaining what their children needed as braille users in a general education classroom. Along with the TVI, primary caregivers expressed a sense of frustration in communicating the unique needs of children who were blind. "We went through a phase where we were having trouble with the school itself on having a braille teacher in there and making it so everything was adapted so she could participate. I think we spent the first half of the year trying to get the teacher to have the proper materials so she could be included." Another primary caregiver relayed "I do think [child's name] spent a fair amount of time in the hallway learning the braille instead of being in the classroom with the other kids and part of that was the classroom teacher didn't like the noise of the brailler, thought it was disruptive to other students, distracting." As a result, primary caregivers expressed frustration with teachers who were reluctant to learn and include adaptations and accommodations in the classroom.

\section{Interpretation of Findings}

This study was completed in two phases, one extensive document review, second, semi structured interviews. Both phases provided results and themes to answer the 
primary research question: How is the construct of school readiness defined for children who are blind and use braille as their primary medium? Additional research questions included: (a)What is the current focus of instruction for children who are blind in preschool to prepare them for kindergarten? (b) What is the role of the TVI and primary caregiver in preparing students for kindergarten? (c) What do TVIs and primary caregivers identify as the barriers to school readiness for children who are blind? (d) What do TVIs and primary caregivers see as essential to preparing children who are blind for kindergarten? The following is the interpretation of the findings based on both phases of this study.

\section{How is the Construct of School Readiness Defined for Children who are Blind and} use Braille as their Primary Medium? Analysis of documents and interviews with key stakeholders suggest school readiness for children who are blind was primarily focused on braille readiness, specifically instruction in tactually accessing the braille code and orientation and mobility. There was a secondary focus on mathematics specifically in counting and cardinality. In addition, there was a third area of focus on gross motor skills including orientation and mobility. When data was reviewed from phase one, $34 \%$ of goals and objectives were in the primary domains of Literacy and Mathematics.

Following this, 29\% was in the primary domain of Perceptual, Motor, and Physical Development. In addition, when TVIs were interviewed, they expressed primary focus on the areas of braille readiness and orientation and mobility.

What is the Current Focus of Instruction for Children who are Blind in Preschool to Prepare them for Kindergarten? 
Primary caregivers and TVIs were in agreement there is a need for increased time in instruction to prepare students who are blind for kindergarten. There was alignment between how TVIs and primary caregivers define kindergarten readiness and the focus of instruction for students who are blind and use braille as a primary medium. Both expressed the need to prepare students to participate academically as well as development independent travel skills. Both shared concerns that children who are blind are developmentally behind their sighted peers. It should be noted, however, that two of the three primary caregivers interviewed suggested they needed to "push" for braille instruction in the preschool years and felt their children started kindergarten significantly behind their sighted peers. The data suggests that TVIs focus on tactile discrimination skills and the braille code, whereas, primary caregivers focus on alphabet knowledge and traditional skills of reading.

\section{What is the Role of the TVI and Primary Caregiver in Preparing Students for}

\section{Kindergarten?}

The role of the TVI and primary caregiver is unique for children who are blind. In an analysis of documents, education service is delivered primarily by a TVI or orientation and mobility specialist. While students received services from special education providers and related services including occupational or physical therapy or, speech/language therapy, the role of the TVI is the most significant educational component for specially designed instruction. It should be noted consultation, coaching, and training of general education staff was a significant component of the role and time of the TVI. TVIs expressed the need for significant time to coach and train general 
education personnel in techniques and strategies to support access to the general education curriculum. In addition, identifying and implementing accommodations, modifications and adaptation of the educational environment was primary the responsibility of the TVI.

The role of the primary caregiver varied as it does for sighted children. Parents interviewed did have a common concern of being unfamiliar with the braille code and not truly knowing how to read to their child. There was also a common theme of feeling lost and disempowered in supporting their child in the school environment. A key theme emerged in the need for consistent and comprehensive communication between the TVI, general education personnel and the primary caregiver. Similar to other primary caregivers of children with disabilities, there was an identified need for advocacy beyond what is needed for sighted children. Primary caregivers expressed the need to be in communication with all facets of their child's education experience

\section{What do TVIs and Primary Caregivers Identify as Barriers to School Readiness for Children who are Blind? What do TVIs and Primary Caregivers see as Essential to Preparing Children who are Blind for Kindergarten?}

Based on an analysis of the data, further examination of the direct instruction time for children who are blind is needed. There was a clear consensus from TVIs and primary caregivers that the time provided is simply not enough given the unique needs of children who are blind. This includes time for direct instruction, consultation, coaching for general education staff and continued support for families and primary caregivers. 
However, when it came to strategies to increase student time, TVIs expressed frustration with existing caseloads and staff shortages.

My hypothesis was education services for children who are blind and use braille, both the type and intensity needed, may not align with the current construct of school readiness for sighted children. Based on an examination of critical documents and interviews with key participants, this hypothesis is supported based on the student data sets examined from some parts of Oregon. A review of documents clearly showed domains and sub domains of the Head Start Early Learner Outcome Framework (USDHHS, 2015) not addressed in preschool for children who are blind and use braille. In addition, a common theme for both teachers of the visually impaired and primary caregivers was the need to increase time due the unique and extensive needs of children who are blind. Sighted children have the benefit of incidental learning while children who are blind need explicit instruction in the primary domains of the Head Start Early Learner Outcome Framework as well as the Expanded Core Curriculum (Hatlen, 1996). There is simply a lot to learn when you cannot access the world with vision.

When interpreting the analysis of IFSP and IEP goals and objectives as well as service time, it is important to note that these documents are the result of an educational team. In this study it was not possible to interview other members of the team to ascertain their instructional focus for the child. It is also important to note the importance of the Learning Media Assessment for children who are blind and tactile learners. The decision of the learning media is the process of the IFSP/IEP team, however, often led by the TVI. Some times there is reluctance on the part of team members to select braille as a 
primary learning media. There are a variety of circumstances that may lead to this delay of determination such as the unique characteristics of the eye condition or presence of additional disabilities. This may be the result of the misconception that braille is a slow and cumbersome reading method; other times, this may be a result of fear and the grieving process. As stated above, a delay in determining the learning media for a child delays the instructional focus and process in kindergarten readiness skills.

Dedication and passion to children who are blind and visually impaired was evident throughout this study on the part of teachers of the visually impaired and primary care givers. An additional common theme was the uniqueness of children who are blind and use braille. Children who fit this criterion are indeed a small population which can result in children, families and teachers feeling isolated and without adequate resources, colleagues, peer models, and family support for primary caregivers.

\section{Limitations of Study}

There were many important findings in this study, however, we must note the limitations of this study. The first limitation to note is all information was based on Oregon services. The intention of this study was not generalization but rather an exploration of services for visually impaired students based in Oregon. Therefore, the results should not be generalized beyond Oregon.

Another limitation was the small sample size. Krathwohl (2009) notes that larger sample sizes are preferable because it is easier to generalize results. However, as stated in Chapter 3, the intention of this study was not to generalize but to inform regional programs of possible areas to focus or change services to children who are blind. A 
small sample size was used for both the document review as well as interviews for participants. A request for documents was sent to the eight regional providers, however, ten documents sets were returned from only four of the eight regional programs (50\%). This may suggest that other regional programs did not have children who fit the criteria. Or there was a reluctance to share the work of the regional program or confidential student information.

A limitation of the study was the lack of diversity of TVI participants from all regions in Oregon. I interviewed five teachers of the visually impaired from three regions, one of which is the regional program where I am employed. Despite efforts to identify primary care participants, only three agreed to be interviewed. A limitation of this study was that all of the primary caregiver interviews were from one regional program. The lack of representation of TVIs and primary caregivers across Oregon could bias the results and lead to the results being specific to one regional program versus services in the state. A final limitation is that this study did not address the Ecological and Dynamic Model of Transition (Rimm-Kaufman \& Pianta, 2000) in its entirety. This research wasfocused on teachers of the visually impaired and family (primary caregivers), but did not examine neighborhood, peers and the nature of the relationships over time. These limitations might be addressed in the future by using a case study method of research or a longitudinal study following children who are blind over a period of three years as they attend preschool and transition to kindergarten.

In conclusion, data analyses revealed instructional focus for children who are blind and use braille as their primary medium addresses all of the primary domains of the 
Head Start Early Learner Outcome Framework (USDHHS, 2015) but is inconsistent in addressing the sub domains. This is consistent with the notion that there is a need for increased time in preschool to addresses additional areas of kindergarten readiness. In addition, eight out nine areas of the expanded core curriculum were addressed in preschool and kindergarten years; there was alignment between the expanded core curriculum and the Head Start Early Learning Outcomes Framework (USDHHS, 2015). TVI and primary caregiver voices provided evidence of awareness of the need for increased time and focus on the whole child in addition to braille readiness. TVIs also expressed the need overall for additional professional development in the area of kindergarten readiness and changing expectations and standards.

In phase one of this study, I used data analyses to identify the goals and objectives that are the focus of instruction for children who are blind in preschool and kindergarten. I used this data to analyze services children who are blind receive in early childhood special education and school age services. In phase two I used data analysis to understand how TVIs and primary caregivers of children who are blind construct the notion of kindergarten readiness. Phase one of this study supported the hypothesis that the educational focus for children who are blind and read braille is limited and not comprehensive of the full Head Start Early Learner Outcome Framework (USDHHS, 2015) sub domains. It may be further research is needed to determine if the Head Start Early Learner Outcomes Framework (USDHHS, 2015) is an accurate construct for readiness for children who are blind and are tactile learners. Additional research may be needed to examine how kindergarten readiness relates to the placement of the child in 
general or specialized classrooms, as well as the involvement of the general educator. Interviews with key participants confirmed a primary reason for this finding may be a lack of time and flexibility in serving students. The data also indicates in the field of vision impairment, for both TVIs and primary care givers, the construct of kindergarten readiness mirrors research in the field and focuses primarily on braille readiness, specifically the focus on learning tactile skills and the braille code configuration. 


\section{Chapter 5: Conclusion}

This study set out to determine the answer to the primary research question: How is the construct of school readiness defined for children who are blind, are tactile learners and use braille as their primary learning medium. This research was propelled by a compelling problem, children who are blind may not be ready to participate and succeed in the general education kindergarten classroom. If children who are blind have the necessary school readiness skills, including academic, social, and behavioral skills when entering kindergarten, then they will be better prepared to participate fully in the academic classroom.

A thorough review of the literature noted kindergarten readiness for children who are blind has predominately focused on the braille readiness not specifically addressing additional academic, social, and behavioral skills. While kindergarten readiness has been investigated for children who are blind and use braille, my review of the literature indicated there were no studies on alignment with Head Start Early Learner Outcome Framework (USDHHS, 2015). Additionally, this study was completed through the lens of the Rimm-Kaufman and Pianta's (2000) Ecological and Dynamic Model of Transition, where family and teachers are critical in both preschool and kindergarten. This research focused on child characteristics of school readiness in the context of preschool and the context of the kindergarten classroom from the perspective of family and teachers of the visually impaired. Along with the primary research question, the following research questions were included in my research. 
1. What is the current focus of instruction for children who are blind in preschool to prepare them for kindergarten?

2. What is the role of the TVI and primary caregiver in preparing students for kindergarten?

3. What do TVIs and primary caregivers identify as the barriers to school readiness for children who are blind?

4. What do TVIs and primary caregivers see as essential to preparing children who are blind for kindergarten?

As noted in Chapter 2, the lack of research specific for children who are blind and readiness for kindergarten speaks to the significance and importance of this research. After a thorough review of the literature and using the Ecological and Dynamic Model of Transition (Rimm-Kaufman \& Pianta, 2000), school readiness for the purposes of this study was compared to the developmental domains included in the Head Start Early Learning Outcomes Framework (USDHHS, 2015) identified by Oregon as early learning standards for all children ages three to five. Readiness skills were further defined by the concepts assessed in the Oregon Kindergarten Readiness Assessment given to all incoming kindergarteners prior to beginning school. School readiness for children who are blind was further examined to include the Expanded Core Curriculum (Hatlen, 1996) as defined as a set of additional skills and knowledge which children who are blind need in addition to core curriculum. School readiness was not a set of required skills for attendance in kindergarten and not a means to prevent or delay children with disabilities from attending general education, rather, readiness were set of skills identified that 
enabled a child's successful transition into public school and creates a foundation for their later school success.

\section{Synthesis of Findings}

The following section synthesizes findings of this research including an examination of the field of education for children who are blind as well as identification of larger themes.

\section{Phase One}

This study has shown that when goals and objectives were analyzed for alignment with the Head Start Early Learner Outcomes Framework (USDHHS, 2015) and the Expanded Core Curriculum (Hatlen, 1996), there were goals and objectives representing all preschool domains: Approaches to Learning, Social and Emotional Development, Language and Communication, Literacy, Mathematics Development, Scientific Reasoning, and Perceptual, Motor, and Physical Development. There was less consistent alignment with Head Start sub-domains. The lack of focus on sub-domains speaks to the need for more comprehensive instructional focus using the theoretical framework of the Ecological and Dynamic Model of Transition (Rimm-Kaufman \& Pianta, 2000). For example, a lack of goals and objectives in Cognitive Self-Regulation (Executive Functioning Skills) may be detrimental to the successful inclusion in a typical kindergarten, particularly in that kindergarten teachers report the need for social skills and social behaviors a higher priority to readiness than academic concerns (Lin et al., 2003). A focus on the whole child would result in instructional practices that recognize the importance of such areas as Initiative and Curiosity, Creativity, Cognitive Self Regulation, Emotional Functioning and Sense of Identity and Belonging. An additional 
focus on comprehensive academics would lead to more instruction focused on phonetic awareness, comprehension of text structure, mathematics operations and algebraic thinking and measurement. While there was alignment with the ECC the narrow focus of goals suggests that TVIs would benefit from additional curriculum and instructional guides that provide breadth and variety of instructional goals and objectives.

A second major finding of phase one was the discrepancy in instructional time provided in preschool versus kindergarten. This discovery was also noted in phase two of this study when interviewing key participants in the education of children who are blind. While TVIs report the need for additional instructional time in early childhood, they report barriers such as caseloads demands, paperwork, meetings, and the need for extensive environmental accommodations and material preparation. Caseload analysis to include recommended time for tactile learners in preschool as well as and structure of specially designed instruction and access to quality preschool environments should be the focus of future examination and research.

\section{Phase Two}

Semi-structured interviews with TVIs and primary caregivers, led to support of the findings from phase one of this study. TVIs were in agreement that children who are blind and use braille are developmentally behind their sighted peers and universally agreed more time in instruction should be provided. While TVIs discussed the need to educate the whole child, the predominant focus continued to be braille readiness. There was also universal agreement on the importance of primary caregiver involvement. Again, noting challenges of time constraints in providing direct instruction to the families 
as well as coaching and training.

Primary caregivers universally reported the need for additional support, coaching, land guidance in the use of braille and supporting their child's educational progress. Support, coaching, and guidance could be in the form of community resources, connections with other families with children who are blind or materials and resources to support instructional goals in the home environment. They also universally reported the importance and need for consistent communication from educational providers including general education teachers as well as TVIs.

Overall, the study suggests that TVIs definition of the construct of kindergarten readiness does not reflect current research based on children who are sighted. The current emphasis on heightened academic rigor (Rimm-Kaufman, 2004) and heightened expectations of social and behavioral competence (Lin et al., 2003) were not expressed by TVIs or primary caregivers. This study has shown there is a need for increased professional development for TVIs in particular, regarding the increased expectations for kindergarten as well as the development of a more rigorous preschool curriculum, instruction and accountability for educational outcomes.

\section{Situated in Larger Context}

Kindergarten is a critical milestone for all students including those with identified disabilities, specifically blindness. For all children, there is an increased focus on school readiness as children enter kindergarten; school readiness broadly defined to include literacy, mathematical and cognitive skills, social, emotional, behavioral and physical development (Workman et al., 2014; Duncan et al., 2007; Farran, 2011; Kagan \& Rigby, 
2003; Konold \& Pianta, 2005). Given the unique challenges for students who are blind and their families in K-12 public school, school readiness in the transition to and preparation for kindergarten is even more critical to their future academic success as well as to the successful relationship between the family and public school (Daley et al., 2011).

Recent reauthorization Elementary and Secondary Education Act (ESEA) to Every Student Succeeds Act (ESSA) has included early childhood education in a more robust way. The new bill explicitly states providing early education programs are an allowable use of Title I funds prior to kindergarten and encourage planning for transition form pre-k programs to elementary schools. ESSA also allows for Title II funds (funds to prepare, train and recruit highly qualified educators) to be used for early educators. This would allow districts and programs to provide joint professional development for early childhood special educators and kindergarten teachers to further align curriculum, learning activities and expectations for children entering kindergarten. ESSA also includes a "Preschool Development Grant" program which would allow 18 states to develop or expand access to high-quality pre-k programs for four year olds from low income families. The program requires quality indicators including full day prekindergarten programs and lead teacher compensated comparably to K-12 teachers. The purpose of these grants is specifically to improve collaboration and coordination among existing early education programs and school-based services.

Oregon specifically has directed additional investment in preschool programs in the state through the passage of House Bill 3380 that directed the Early Learning 
Division to invest $\$ 16$ million in the creation of quality preschool services across the state. The program is called Preschool Promise and is administered through the early learning hubs. The expectations for these preschool programs are to provide high quality programs which maintain culturally responsive teaching methods, family engagement strategies, assessments, curricula, and professional development linked to one another and the state's comprehensive early learning standards.

While there is no question there is an increased emphasis on early childhood education standards including high quality instruction aligned with early elementary expectations (Rimm-Kaufman, 2004), it is not clear that TVIs are receiving or participating in professional development aligned with these changes. It is critical for this population of teachers to receive high quality professional development in current research and best practice in early childhood to improve their teaching, to understand increased expectations for preschool and early elementary children and to be able to coach and support parents and families as they partner to prepare their children to enter public school.

\section{Implications}

This study has several implications regarding policy and practice in the field of education for blind and visually impaired students. The small sample size of this study makes it unwise to make generalizations, however, I believe there remain implications for services to children who are blind and use braille as their primary medium

Based on the findings and outcomes of this study, professional development for TVIs should include current research and trends in early childhood education. In 
addition, professional development specifically in the Head Start Early Learner Outcome Framework (USDHHS, 2015) as well as the Kindergarten Readiness Assessment and best practice in kindergarten transition should be ongoing for TVIs. Based on interviews with TVIs, they have not been included as a specific group in this type of professional development; this is significant for regional programs and a consideration for future planning for professional development.

There is also a recommendation for additional professional development in the administration, interpretation, and presentation of the Learning Media Assessment. Given that two out of three students in phase two (primary caregiver interview) experienced indecision around their primary learning medium, it suggests there was indecision and misunderstanding of the process. Learning media assessment was not part of this study, however, the experiences of primary caregivers and TVIs when children are not taught braille from the beginning speaks to the need for further research and guidance in this area of assessment. One TVI suggested:

We don't do our Functional Vision Assessment, Learning Media Assessment early enough to get a good idea of what students really should be involved in tactile readiness and what kids need to be a good braille reader. I think there is no harm coming from teaching braille to a student who may become a print reader but there's a lot of harm that comes form those kids not having any understanding of braille, taught as a sighed student when they are not going to be a print reader, that's where the big problems happen. Because they can see an object, they presume they can read but those are very different things. (TVI interview) 
Oregon does not require a Learning Media Assessment until age three when the child enters Early Childhood Special Education (ECSE). Researching the timing of these critical assessments may lead to different outcomes regarding learning media assessments determinations made earlier in a child's education. Presumably, earlier decisions regarding braille would allow families and caregivers time to adjust and create an environment to encourage the alternative format. During the period of this study, the American Printing House for the Blind (APH) has been revising the primary curriculum for students learning braille, Building on Patterns. The original intent was to revise the kindergarten level, however, after looking at multiple state standard for pre-kindergarten, it was determined there was a need for a prekindergarten level curriculum. In the development of the Building on Patterns kindergarten level, the authors surveyed TVIs to ask: What is taught in the kindergarten curriculum that should be taught in prekindergarten early literacy program? The top three responses were phonemic awareness and phonics, the alphabet, or an introduction to the alphabet contractions and at least the first 12 lessons of the kindergarten curriculum should be taught earlier (Blaylock et al., 2015). Additional skills mentioned by TVIs were tracking and reading with both hands, rhyming, introduction to the braille cell, basic punctuation, vocabulary, concept development, listening comprehension, tactile identification and spatial awareness (Blaylock et al., 2015). The result of the examination of early kindergarten entry standards determined children who are blind are entering kindergarten with sighted peers who recognize letters and numbers as well as read and write their names (Blaylock et al., 2015). Components of the pre-kindergarten curriculum will including reading and 
writing braille letters and numbers, reading and writing simple continuous text, interactive read-a-louds, comprehension and vocabulary, phonological awareness, concept development and tactile skills (Blaylock et al., 2015).

Ecological and Dynamic Model of Transition. Additional research is needed to determine how TVIs and primary caregivers define the construct of kindergarten readiness for children who are blind and use braille as their primary medium through the lens of the whole child. There is no question kindergarten readiness is a construct fully integrated into our educational frameworks. Kindergarten readiness examined through the framework of the Ecological and Dynamic Model of Transition (Rimm-Kaufman \& Pianta, 2000) would provide the field of vision impairment with research and evidence based practices to increase educational outcomes for children who are blind. The strength of the Rimm-Kaufman and Pianta (2000) model is the comprehensive examination of combined influences environmental contexts including peers, teachers, neighborhood and family. My research focused on the direct effects of the teacher of the visually impaired and family and outcomes based on the Head Start Early Learner Outcomes (USDHHS, 2015) and Expanded Core Curriculum (Hatlen, 1996) frameworks, however, an additional and central distinction of the Ecological and Dynamic Model of Transition is the emphasis on the development of relationships over time (RimmKaufman \& Pianta, 2000). Given the unique role of the teacher of the visually impaired, future examination of the impact of this relationship could have further implications for school readiness for children who are blind.

Additional considerations for future research should include larger scale studies of 
children who are blind and use braille as their primary medium and the focus of their instruction. This would include a larger scale study to determine instructional time and focus for children as well as the nature of intervention and level of family engagement. While there is research and a professional consensus in the field on appropriate levels of instruction services for braille (Koenig \& Holbook, 2000), again, the focus is on braille literacy and not the whole child. In addition, interviewees report programs are not providing the necessary personnel to support the recommended levels of service time in early childhood services. Research to determine specific skill sets within the Expanded Core Curriculum (Hatlen, 1996) as well as alignment with Head Start Early Learning Outcome Framework (USDHHS, 2015) will guide educational practice and increase educational outcomes and success for children who are blind.

There is no question the transition from preschool to kindergarten and a child's readiness for that environment has long lasting educational implications. The topic of kindergarten readiness has limited research in the field of vision impairment. Providing teachers of the visually impaired and primary caregivers with the tools, knowledge and strategies to support children who are blind as they transition to kindergarten will increase educational outcomes and opportunities. 


\section{References}

Alper, M. (2012). Promoting emerging new media literacies among young children with blindness and visual impairments. Digital Culture \& Education, 4(3), 243- 256. http://www.digitalcultureandeducation.com/uncategorized/dce1077_allen_2012_h tml-2/

American Printing House for the Blind (APH), “Annual report 2014: Distribution of eligible students based on the federal quota census of January 7, 2013 (Fiscal year 2014).” Retrieved from http://www.aph.org/federal-quota/distribution-2014/

Andersen, E. S., Dunlea, A., \& Kekelis, L. S. (1984). Blind children's language: Resolving some differences. Journal of Child Language, 11(3), 645-664.

Apkarian-Stielau, P., \& Loomis, J. M. (1975). A comparison of tactile and blurred visual form perception. Perception \& Psychophysics, 18(5), 362-368. doi: $10.3758 / \mathrm{BF} 03211213$

Aunola, K., Leskinen, E., Lerkkanen, M. K., \& Nurmi, J. E. (2004). Developmental dynamics of math performance from preschool to grade 2. Journal of Educational Psychology, 96(4), 699-713. doi: 10.1037/0022-0663.96.4.699

Bailey, L. B. (2014). A review of the research: Common core state standards for improving rural children's school readiness. Early Childhood Education Journal, 42(6), 389-396. doi: 10.1007/s10643-013-0621-6

Baroody, A. E., \& Diamond, K. E. (2012). Links among home literacy environment, literacy interest, and emergent literacy skills in preschoolers at risk for reading 
difficulties. Topics in Early Childhood Special Education, 32(2), 78-87. doi: $10.1177 / 0271121410392803$

Barron, R. W., \& Baron, J. (1977). How children get meaning from printed words. Child Development, 48(2), 587-594. doi: 10.2307/1128657

Bassett, R., Beagan, B. L., Ristovski-Slijepcevic, S., \& Chapman, G. E. (2008). Tough teens the methodological challenges of interviewing teenagers as research participants. Journal of Adolescent Research, 23(2), 119-131. doi: $10.1177 / 0743558407310733$

Bates, E. (1979). Intentions, conventions and symbols. In E. Bates, L. Benigni, I., Bretherton, L., Camaioni, \& V., Volterra (Eds.), The emergence of symbols: Cognition and communication in infants (pp. 33-68). New York, NY: Academic Press.

Bedny, M., \& Saxe, R. (2012). Insights into the origins of knowledge from the cognitive neuroscience of blindness. Cognitive Neuropsychology, 29(1-2), 56-84. doi: $10.1080 / 02643294.2012 .713342$

Bigelow, A. (1987). Early words of blind children. Journal of Child Language, 14(01), 47-56. doi: 10.1017/S0305000900012721

Bigelow, A. (1990). Relationship between the development of language and thought in young blind children. Journal of Visual Impairment and Blindness, 84, 414-419.

Blair, C. (2002). School readiness: Integrating cognition and emotion in a neurobiological conceptualization of children's functioning at school entry. American 
SCHOOL READINESS: CHILDREN WHO ARE BLIND

Psychologist, 57(2), 111-127. http://dx.doi.org.proxy.lib.pdx.edu/10.1037/0003066X.57.2.111

Blaylock, L, Croft, J., Dilworth, K., Ferrell, K., Holbrook, C., Senft-Graves, C., . . . Wingell, R. (November, 2015). Prekindergarten building on patterns: Lesson learned. Presented at Getting in Touch with Literacy, Albuquerque, NM.

Blyth, E., \& Milner, J. (1994). Exclusion from school and victim-blaming. Oxford Review of Education, 20(3), 293-306.

Bradley, L., \& Bryant, P. (1991). Phonological skills before and after learning to read. In S. Brady \& D. Shankweiler (Eds.), Phonological processes in literacy: A tribute to Isabelle Y. Liberman (pp. 37-45). New York, NY: Routledge.

Brennan, S. A., Luze, G. J., \& Peterson, C. (2009). Perceptions of professional support for the emergent literacy of young children with visual impairments. Journal of Visual Impairment \& Blindness, 103(10), 694-704.

Bricker, D. D., Squires, J., \& Mounts, L. (1999). Ages \& stages questionnaires: A parentcompleted, child-monitoring system. Baltimore, MD: Paul H. Brookes.

Brown, C., \& Bour, B. (1986). Movement analysis and curriculum for visually impaired preschoolers: A resource manual for the development and evaluation of special programs for exceptional students. Tallahassee, FL: Bureau of Education of Exceptional Students, Florida Department of Education.

Brown, R., Hobson, R. P., Lee, A., \& Stevenson, J. (1997). Are there “Autistic $\square$ like" features in congenitally blind children? Journal of Child Psychology and Psychiatry, 38(6), 693-703. doi: DOI: 10.1111/j.1469-7610.1997.tb01696.x 
Brown, D., Simmons, V., \& Methvin, J. (1978). The Oregon Project for Visually Impaired and Blind Preschool Children: Skills Inventory. Medford, OR: Jackson County Education Service District

Burns, M. S., Donovan, M. S., \& Bowman, B. T. (Eds.). (2000). Eager to Learn: Educating Our Preschoolers. Washington, DC: National Academies Press.

Burgess, S. R., Hecht, S. A., \& Lonigan, C. J. (2002). Relations of the home literacy environment (HLE) to the development of reading-related abilities: A one-year longitudinal study. Reading Research Quarterly, 37(4), 408-426.

Carlton, M. P., \& Winsler, A. (1999). School readiness: The need for a paradigm shift. School Psychology Review, 28, 338-352.

Celeste, M. (2006). Play behaviors and social interactions of a child who is blind: In theory and practice. Journal of Visual Impairment \& Blindness, 100(2), 75-90.

Chall, J. (1983). Stages of reading development. New York, NY: McGraw-Hill.

Charmaz, K. (2014). Constructing grounded theory ( $2^{\text {nd }}$ ed.). Thousand Oaks, CA: Sage.

Chiang, Y. P., Bassi, L. J., \& Javitt, J. C. (1992). Federal budgetary costs of blindness. The Milbank Quarterly, 70(2), 319-340. doi: 10.2307/3350062

Chien, N., Howes, C., Burchinal, M., Pianta, R., Ritchie, S., Bryant, D.,...Barbarin, O. (2010). Children's classroom engagement and school readiness gains in prekindergarten. Child Development, 81(5), 1534-1549. doi: 10.1111/j.14678624.2010.01490.x 
Cho, J. Y., \& Lee, E. (2014). Reducing confusion about grounded theory and qualitative content analysis: Similarities and differences. The Qualitative Report, 19(64), 120.

Clarke, A. T., \& Kurtz-Costes, B. (1997). Television viewing, educational quality of the home environment, and school readiness. The Journal of Educational Research, 90(5), 279-285. doi: 10.1080/00220671.1997.10544584

Conroy, P. W. (2007). Paraprofessionals and students with visual impairments: Potential pitfalls and solutions. $R E$ : view, 39(2), 43-55.

Corbin, J., \& Strauss, A. (1994). Grounded theory methodology: An overview. In N. K. Denzin \& Y. S. Lincoln (Eds.), Handbook of qualitative research (pp. 273-285). Thousand Oaks, CA: Sage.

Craig, C. J. (1996). Family support of the emergent literacy of children with visual impairments. Journal of Visual Impairment and Blindness, 90, 194-200.

Creswell, J. W. (2012). Qualitative inquiry and research design: Choosing among five approaches. Thousand Oaks, CA: Sage.

Cutsforth, T. D. (1951). The blind in school and society: A psychological study. New York, NY: American Foundation for the Blind.

Daley, T., Munk, T., \& Carlson, E. (2011). A national study of kindergarten transition practices for children with disabilities. Early Childhood Research Quarterly, 26, 409-419. doi: 10.1016/j.ecresq.2010.11.001

D'Allura, T. (2002). Enhancing the social interaction skills of preschoolers with visual impairments. Journal of Visual Impairment \& Blindness, 96(8), 576-584. 
Denham, S. A. (2006). Social-emotional competence as support for school readiness: What is it and how do we assess it? Early Education and Development, 17(1), 5789. doi: 10.1207/s15566935eed1701_4

Denham, S. A., \& Brown, C. (2010). Plays nice with others: Social-emotional learning and academic success. Early Education and Development, 21(5), 652-680. doi: $10.1080 / 10409289.2010 .497450$

Denzin, N. K., \& Lincoln, Y. S. (2011). Introduction: The discipline and practice of qualitative research. The Sage handbook of qualitative research (4th ed., pp. 119). Thousand Oaks, CA: Sage.

Dockett, S., \& Perry, B. (2002). Who's ready for what? Young children starting school. Contemporary Issues in Early Childhood, 3(1), 67-89. doi: 10.2304/ciec.2002.3.1.9

Dodd, B., \& Conn, L. (2000). The effect of braille orthography on blind children's phonological awareness. Journal of Research in Reading, 23(1), 1-11. doi: $10.1111 / 1467-9817.00098$

Duncan, G. J., Dowsett, C. J., Claessens, A., Magnuson, K., Huston, A. C., Klebanov,..., Japel, C. (2007). School readiness and later achievement. Developmental Psychology, 43(6), 1428. doi: http://dx.doi.org.proxy.lib.pdx.edu/10.1037/00121649.43.6.1428.supp.

Dunlea, A. (1989). Vision and the emergence of meaning: Blind and sighted children's early language. Cambridge, MA: University Press. 
Dunn, J. (1988). The beginnings of social understanding. Cambridge, MA: Harvard University Press.

Early, D. M., Pianta, R. C., Taylor, L. C., \& Cox, M. J. (2001). Transition practices: Findings from a national survey of kindergarten teachers. Early Childhood Education Journal, 28(3), 199-206. doi: 10.1023/A:1026503520593

Edwards, C. P., \& Whiting, B. B. (1988). Children of different worlds. Cambridge, MA: Harvard University Press.

Emerson, R. W., Holbrook, M. C., \& D’Andrea, F. M. (2009). Acquisition of literacy skills by young children who are blind: Results from the ABC Braille Study. Journal of Visual Impairment \& Blindness, 103(10), 610-624.

Entwisle, D. R., \& Alexander, K. L. (1996). Family type and children's growth in reading and math over the primary grades. Journal of Marriage and the Family, 58(2), 341-355. doi: $10.2307 / 353500$

Erickson, K. A., \& Hatton, D. (2007). Expanding understanding of emergent literacy: Empirical support for a new framework. Journal of Visual Impairment \& Blindness, 101(5), 261-277.

Erickson, W., Lee, C., \& Von Schrader, S. (2013). Disability Statistics from the 2011 American Community Survey (ACS). Ithaca, NY: Cornell University Employment and Disability Institute (EDI).

Erwin, E. J. (1993). Social participation of young children with visual impairments in specialized and integrated environments. Journal of Visual Impairment \& Blindness, 87(5), 138-142. 
Erwin, E. J. (1994). Social competence in young children with visual impairments. Infants \& Young Children, 6(3), 26-33.

Farran, D. C. (2011). Rethinking school readiness. Exceptionality Education International, 21(2), 5-15.

Ferrell, K. A. (1986). Working with infancy and early childhood. In G. T. Scholl (Ed.), Foundations of education for blind and visually handicapped children and youth: Theory and practice, (pp. 265-274). New York, NY: American Foundation for the Blind.

Ferrell, K. A., Trief, E., Deitz, S. J., \& Bonner, M. A. (1990). Visually Impaired Infants Research Consortium (VIIRC): First-year results. Journal of Visual Impairment \& Blindness, 84(8), 404-410.

Fitch, F. (2003). Inclusion, exclusion, and ideology: Special education students' changing sense of self. The Urban Review, 35(3), 233-252. doi: 10.1023/A:1025733719935

Fitzpatrick, C., McKinnon, R. D., Blair, C. B., \& Willoughby, M. T. (2014). Do preschool executive function skills explain the school readiness gap between advantaged and disadvantaged children? Learning and Instruction, 30, 25-31. doi:10.1016/j.learninstruc.2013.11.003

Fitzpatrick, C., \& Pagani, L. S. (2012). Toddler working memory skills predict kindergarten school readiness. Intelligence, 40(2), 205-212. doi:10.1016/j.intell.2011.11.007

Flavell, J. H. (1988). The development of children's knowledge about the mind: From cognitive connections to mental representations. In J. Astington, P. Harris, \& D. 
Olson (Eds.), Developing theories of mind (pp. 244-267). New York, NY: Cambridge University Press.

Foulke, E., Amster, C. H., Nolan, C. Y., \& Bixler, R. H. (1962). The comprehension of rapid speech by the blind. Exceptional Children, 29(3), 131-141.

Fowler, S. A., Schwartz, I., \& Atwater, J. (1991). Perspectives on the transition from preschool to kindergarten for children with disabilities and their families. Exceptional Children, 58(2), 136-145.

Fraiberg, S. (1977). Insights from the blind: Comparative studies of blind and sighted infants. New York, NY: Basic Books.

Fraiberg, S., \& Adelson, E. (1977). Self-representation in language and play: observations of blind children. In S. Fraiberg, Insights from the blind: Comparative studies of blind and sighted infants. (pp. 248-270). New York, NY: Basic Books.

Fraiberg, S., Smith, M., \& Adelson, E. (1969). An educational program for blind infants. The Journal of Special Education, 3(2), 121-139. doi: $10.1177 / 002246696900300201$

Gardner, D. P. (1983). A nation at risk. Washington, DC: The National Commission on Excellence in Education, US Department of Education.

Gathercole, S. E., \& Baddeley, A. D. (1990). Phonological memory deficits in language disordered children: Is there a causal connection? Journal of Memory and Language, 29(3), 336-360. doi:10.1016/0749-596X(90)90004-J 
Giangreco, M. F. (2010). One-to-one paraprofessionals for students with disabilities in inclusive classrooms: Is conventional wisdom wrong? Intellectual and Developmental Disabilities, 48(1), 1-13. doi: http://dx.doi.org.proxy.lib.pdx.edu/10.1352/1934-9556-48.1.1

Gill, S., Winters, D., \& Friedman, D. S. (2006). Educators' views of pre-kindergarten and kindergarten readiness and transition practices. Contemporary Issues in Early Childhood, 7(3), 213-227. doi: 10.2304/ciec.2006.7.3.213

Goldstein, L. (2007). Embracing pedagogical multiplicity: Examining two teachers' instructional responses to the changing expectations for kindergarten in U.S. public schools. Journal of Research in Childhood Education, 21, 378-399. doi:10.1080/02568540709594602

Goldstein, P., Warde, B., \& Peluso, P. (2013). Children's readiness gains in publically funded, community-based pre-kindergarten programs for 4 year olds and preschool for 3 year olds. Child \& Youth Care Forum, 42(6), 507-523. doi: $10.1007 / \mathrm{s} 10566-013-9215-0$

Graue, M. E. (1993). Ready for what? Constructing meanings of readiness for kindergarten. Albany, NY: State University of New York Press.

Gredler, G. R. (1992). School readiness: Assessment and educational issues. Brandon, VT: Clinical Publishing Company

Guralnick, M. J., Connor, R. T., Hammond, M., Gottman, J. M., \& Kinnish, K. (1996). Immediate effects of mainstreamed settings on the social interactions and social 
integration of preschool children. AJMR-American Journal on Mental Retardation, 100(4), 359-377.

Haber, L. R., Haber, R. N., \& Furlin, K. R. (1983). Word length and word shape as sources of information in reading. Reading Research Quarterly, 18(2), 165-189. doi: $10.2307 / 747516$

Haines, A. H., Fowler, S. A., Schwartz, I. S., Kottwitz, E., \& Rosenhoetter, S. (1989). A comparison of preschool and kindergarten teacher expectations for school readiness. Early Childhood Research Quarterly, 4, 75-88. doi:10.1016/S08852006(89)90090-2

Hammond, B. (2012, February 05). Sweeping changes to Oregon's early childhood programs would start with small steps. The Oregonian. Retrieved from http://www.oregonlive.com/education/index.ssf/2012/02/sweeping_changes_to_e arly_chil.html

Hanline, M. F., Milton, S., \& Phelps, P. C. (2010). The relationship between preschool block play and reading and math abilities in early elementary school: A longitudinal study of children with and without disabilities. Early Child Development and Care, 180(8), 1005-1017. doi: 10.1080/03004430802671171

Harradine, C. C., \& Clifford, R. M. (1996, April). When are children ready for kindergarten? Views of families, kindergarten teachers, and child care providers. Paper presented at the annual meeting of the American Educational Research Association, New York, NY. 
Hatcher, B., Nuner, J., \& Paulsel, J. (2012). Kindergarten readiness and preschools: Teachers' and parents' beliefs within and across programs. Early Childhood Research \& Practice, 14(2), 1-17.

Hatlen, P. (1996). The core curriculum for blind and visually impaired students, including those with additional disabilities. RE:view, 28(1), 25-32.

Hatton, D. D., Bailey, D. B., Burchinaland, M. R., \& Ferrell, K. A. (1997). Developmental growth curves of preschool children with vision impairments. Child Development, 68(5), 788-806. doi: 10.1111/j.1467-8624.1997.tb01962.x

Hehir, T. (2002). Eliminating ableism in education. Harvard Educational Review, 72(1), $1-33$.

Higgins, L.C. (1973). Classification in congenitally blind children. New York, NY: American Foundation for the Blind.

Hill, N. E. (2001). Parenting and academic socialization as they relate to school readiness: The roles of ethnicity and family income. Journal of Educational Psychology, 93(4), 686-697.

Hoben, M., \& Lindstrom, V. (1980). Evidence of isolation in the mainstream. Journal of Visual Impairment and Blindness, 74(8), 289-92.

Holbrook, M. C., \& Farrenkopf, C. (2009). Guest editors' page. Journal of Visual Impairment \& Blindness, 103(10), 579-581.

Huffman, L. C., Mehlinger, S. L., Kerivan, A. S., Cavanaugh, D. A., Lippitt, J., \& Moyo, O. (2001). Off to a good start: Research on the risk factors for early school problems and selected federal policies affecting children's social and 
emotional development and their readiness for school. Washington, DC: Department of Health and Human Services.

Hughes, C., \& Leekam, S. (2004). What are the links between theory of mind and social relations? Review, reflections and new directions for studies of typical and atypical development. Social development, 13(4), 590-619. doi: 10.1111/j.14679507.2004.00285.x

Hyson, M., \& Biggar, H. (2005). NAEYC's standards for early childhood professional preparation. In M. Zaslow \& I. Martinez-Beck (Eds.), Critical issues in early childhood professional development (pp. 283-308). Baltimore, MD: Paul H. Brookes

Jan, J. E., Robinson, G. C., Scott, E., \& Kinnis, C. (1975). Hypotonia in the blind child. Developmental Medicine \& Child Neurology, 17(1), 35-40. doi: 10.1111/j.1469-8749.1975.tb04954.x

Johnson, J. (2015, February 09). Early learning is key to Kitzhaber's education strategy. The Statesman Journal. Retrieved from http://www.statesmanjournal.com/story/news/2015/02/08/proven-early-educationstill-catching-salem/23013021/

Johnson, L. J., Gallagher, R. J., Cook, M., \& Wong, P. (1995). Critical skills for kindergarten perceptions from kindergarten teachers. Journal of Early Intervention, 19(4), 315-327. doi: 10.1177/105381519501900406

Justice, L. M., Petscher, Y., Schatschneider, C., \& Mashburn, A. (2011). Peer effects in preschool classrooms: Is children's language growth associated with their 
classmates' skills? Child Development, 82(6), 1768-1777. DOI: 10.1111/j.14678624.2011.01665.x

Kagan, S. L. (1990). Readiness 2000: Rethinking rhetoric and responsibility. The Phi Delta Kappan, 72(4), 272-279.

Kagan, S. L., Moore, E., \& Bredekamp, S. (1995). Reconsidering children's early development and learning: Toward shared beliefs and vocabulary. Washington, DC: National Education Goals Panel.

Kagan, S. L., \& Rigby, E. (2003). Policy matters: Setting and measuring benchmarks for state policies: Improving the readiness of children for school:

Recommendations for state policy-a discussion paper for the policy matters project. Washington, DC: Center for the Study of Social Policy.

Katims, D. S., \& Pierce, P. L. (1995). Literacy-rich environments and the transition of young children with special needs. Topics in Early Childhood Special Education, 15(2), 219-234. doi: 10.1177/027112149501500205

Kekelis, L. \& Anderson, E. (1984). Family communication styles and language development. Journal of Visual Impairment \& Blindness, 90(3), 54-65.

Kern, M. L., \& Friedman, H. S. (2009). Early educational milestones as predictors of lifelong academic achievement, midlife adjustment, and longevity. Journal of Applied Developmental Psychology, 30(4), 419-430.

doi:10.1016/j.appdev.2008.12.025

Klein, A., Starkey, P., Clements, D., Sarama, J., \& Iyer, R. (2008). Effects of a prekindergarten mathematics intervention: A randomized experiment. Journal of 
SCHOOL READINESS: CHILDREN WHO ARE BLIND

Research on Educational Effectiveness, 1(3), 155-178. doi:

$10.1080 / 19345740802114533$

Koenig, A. J., \& Farrenkopf, C. (1997). Essential experiences to undergird the early development of literacy. Journal of Visual Impairment and Blindness, 91, 14-24.

Koenig, A. J., \& Holbrook, M. C. (1995). Learning media assessment of students with visual impairments: A resource guide for teachers. Austin, TX: Texas School for the Blind and Visually Impaired.

Koenig, A. J., \& Holbrook, C. (2000). Ensuring high-quality instruction for students in braille literacy programs. Journal of Visual Impairment \& Blindness, 94(11), 677694.

Konold, T. R., \& Pianta, R. C. (2005). Empirically-derived, person-oriented patterns of school readiness in typically-developing children: Description and prediction to first-grade achievement. Applied Developmental Science, 9(4), 174-187. doi: 10.1207/s1532480xads0904_1

Krathwohl, D. R. (2009). Methods of educational and social science research: The logic of methods. Lake Grove, IL: Waveland Press.

Kvale, S. (2008). Doing interviews. Thousand Oaks, CA: Sage.

Lamb, G. (1996). Beginning braille: A whole language-based strategy. Journal of Visual Impairment \& Blindness, 90(3), 184-89.

Landau, B. (1991). Spatial representation of objects in the young blind child. Cognition, 38(2), 145-178. doi:10.1016/0010-0277(91)90050-E 
Landau, B., Gleitman, L. R., \& Landau, B. (2009). Language and experience: Evidence from the blind child. Cambridge, MA: Harvard University Press

Leyser, Y., \& Heinze, T. (2001). Perspectives of children who are visually impaired: Implications for the field. Review, 33(1), 37-48.

Lin, H. L., Lawrence, F. R., \& Gorrell, J. (2003). Kindergarten teachers' views of children's readiness for school. Early Childhood Research Quarterly, 18(2), 225237. doi:10.1016/S0885-2006(03)00028-0

Linder, S. M., Ramey, M., \& Zambak, J. (2013). Predictors of school readiness in literacy and mathematics: A selective review of the literature. Early Childhood Research and Practice, 15(1) Retrieved from http://ecrp.uiuc.edu/v15n1/linder.html.

Litty, C., \& Hatch, A. (2006). Hurry up and wait: Rethinking special education identification in kindergarten. Early Childhood Education Journal, 33, 203-208. doi:10.1007/s10643-005-0048-9

Lloyd, J. E., Irwin, L. G., \& Hertzman, C. (2009). Kindergarten school readiness and fourth grade literacy and numeracy outcomes of children with special needs: A population based study. Educational Psychology, 29(5), 583-602. doi:

$10.1080 / 01443410903165391$

Lowenfeld, B. (1948). Effects of blindness on the cognitive functions of children. The Nervous Child, 7(1), 45-54.

Lowenfeld, B. (1964). Our blind children: Growing and learning with them. Springfield, IL: Thomas. 
Lowenfeld, B. (1981). Berthold Lowenfeld on blindness and blind people: Selected papers. New York, NY: American Foundation for the Blind.

MacComiskey, A.V. (1996). The Braille readiness skills grid: A guide to building a foundation for literacy. Journal of Visual Impairment \& Blindness, 90(3), 19093.

MacCuspie, P.A. (1996). Promoting acceptance of children with disabilities: From tolerance to inclusion. Halifax, Nova Scotia, Canada: Atlantic Provinces Special Education Authority.

Magnuson, K., Meyers, M. K., Ruhm, C. J., \& Waldfogel, J. (2005). Inequality in children's school readiness and public funding. Focus, 24(1), 12-18.

Magnuson, K. A., Ruhm, C., \& Waldfogel, J. (2007). Does prekindergarten improve school preparation and performance? Economics of Education Review, 26(1), 3351. doi:10.1016/j.econedurev.2005.09.008

Mashburn, A. J., \& Pianta, R. C. (2006). Social relationships and school readiness. Early Education \& Development, 17(1), 151-176. doi: 10.1207/s15566935eed1701_7

Maxwell, J. A. (2012). Qualitative research design: An interactive approach. Thousand Oaks, CA: Sage.

May, C. R., \& Campbell, R. M. (1981). Readiness for learning: assumptions and realities. Theory into Practice, 20(2), 130-134. doi: 10.1080/00405848109542941

McAlpine, L. M., \& Moore, C. L. (1995). The development of social understanding in children with visual impairments. Journal of Visual Impairment and Blindness, 89(4), 349-358. 
McClelland, M. M., Acock, A. C., \& Morrison, F. J. (2006). The impact of kindergarten learning-related skills on academic trajectories at the end of elementary school. Early Childhood Research Quarterly, 21(4), 471-490. doi:10.1016/j.ecresq.2006.09.003

McConachie, H. R., \& Moore, V. (1994). Early expressive language of severely visually impaired children. Developmental Medicine \& Child Neurology, 36(3), 230-240. doi: 10.1111/j.1469-8749.1994.tb11836.x

McConnell, S. R., \& Odom, S. L. (1999). A multi-measure performance-based assessment of social competence in young children with disabilities. Topics in Early Childhood Special Education, 19(2), 67-74. doi: $10.1177 / 027112149901900201$

McGonigel, M. J. (1991). Guidelines and recommended practices for the individualized family service plan. Bethesda, MD: Association for the Care of Children's Health. Meisels, S. J. (1999). Assessing readiness. In R. C. Pianta \& M. Cox (Eds.), The transition to kindergarten: Research, policy, training, and practice (pp. 39-66). Baltimore, MD: Paul Brooks.

Mertens, D. M. (2010). Research and evaluation in education and psychology: Integrating diversity with quantitative, qualitative, and mixed methods. Thousand Oaks, CA: Sage.

Mills, A. (1988). Visual handicap. In D. Bishop \& K. Mogford (Eds.), Language development in exceptional circumstances (pp. 150-164). Edinburgh, Scotland: Churchill Livingston. 
Minter, M.E., Hobson, R.P., \& Pring, L. (1991). Recognition of vocally expressed emotion by congenitally blind children. Journal of Visual Impairment \& Blindness, 85, 411-415.

Moretti, F., van Vliet, L., Bensing, J., Deledda, G., Mazzi, M., Rimondini, M., Zimmermann, C., \& Fletcher, I. (2011). A standardized approach to qualitative content analysis of focus group discussions from different countries. Patient Education and Counseling, 82(3), 420-428. doi:10.1016/j.pec.2011.01.005

Mulford, R. (1988). First words of the blind child. In M. D. Smith \& J. L. Locke (Eds.), The emergent lexicon: The child's development of a linguistic vocabulary (pp. 293-338). San Diego, CA: Academic Press.

Murphy, J. L., Hatton, D., \& Erickson, K. A. (2008). Exploring the early literacy practices of teachers of infants, toddlers, and preschoolers with visual impairments. Journal of Visual Impairment and Blindness, 102(3), 133.

National Education Goals Panel (1995). The national education goals report: Building a nation of learners. Washington, DC: U.S. Government Printing Office.

Nelson, K. (1996). Memory development from 4 to 7 years. In A.J. Sameroff \& M. M. Haith (Eds.), The five to seven year shift: The age of reason and responsibility (pp. 141-160). Chicago, IL: The University of Chicago Press.

Neuman, S. B., \& Roskos, K. (2005). The state of state pre-kindergarten standards. Early Childhood Research Quarterly, 20(2), 125-145. doi:10.1016/j.ecresq.2005.04.010 Norris, M., Spaulding, P., \& Brodie, F. (1957). Blindness in children. Chicago, IL: University of Chicago Press. 
O’Brien, M. (1991) Promoting successful transitions into school: A review of current intervention practices. Laurence, KS: Kansas University Early Childhood Research Institute.

Odom, S. L., Buysse, V., \& Soukakou, E. (2011). Inclusion for young children with disabilities A quarter century of research perspectives. Journal of Early Intervention, 33(4), 344-356. doi: 10.1177/1053815111430094

Odom, S. L., \& McLean, M. E. (1996). Early Intervention/Early Childhood Special Education: Recommended Practices. Austin, TX: PRO-ED.

Oleck, J. (2010). Harry Potter. Braille edition released simultaneously with print edition. School Library Journal. Retrieved from http://www. schoollibraryjournal. com/article/CA6462495html

Oregon House Bill 4165 Joint Workgroup. (2012). Recommendations to better coordinate and improve early intervention and early childhood special education services. Retrieved from www.oregon.gov/gov/docs/OEIB/1aaSICCELCHB4165.pdf

Parrish, T., \& Harr, J. (2007). Study of the fiscal and operational efficiency of Oregon's regional and early intervention/early childhood special education programs. Palo Alto, CA: American Institutes for Research.

Parsons, S. (1986). Function of play in low vision children: I. A review of the research and literature. Journal of Visual Impairment \& Blindness, 80(3), 627-630.

Perez-Pereira, M., \& Conti-Ramsden, G. (2013). Language development and social interaction in blind children. Hove, UK: Psychology Press. 
Perkins, K., Columna, L., Lieberman, L., \& Bailey, J. (2013). Parents’ perceptions of physical activity for their children with visual impairments. Journal of Visual Impairment \& Blindness, 107(2), 131-142.

Pestalozzi, J. H., \& Cooke, E. (Eds.). (1898). How Gertrude teaches her children: An attempt to help mothers to teach their own children and an account of the method. Syracuse, NY: CW Bardeen.

Peterson, N. L. (1987). Early intervention for handicapped and at-risk children: An introduction to early childhood-special education. Denver, CO: Love Publishing Company.

Peth-Pierce, R. (2000). A good beginning: Sending America's children to school with the social and emotional competence they need to succeed. Bethesda, MD: National Institute of Mental Health.

Piaget, J. (1952). The origins of intelligence in children. New York, NY: International Universities Press.

Piaget, J. (1955). The language and thought of the child. New York, NY: Meridian Press.

Pianta, R. C., \& Walsh, D. J., (1996). High-risk children in schools: Constructing sustaining relationships. New York, NY: Routledge.

Pierce, C. D., \& Bruns, D. A. (2013). Aligning components of recognition and response and response to intervention to improve transition to primary school. Early Childhood Education Journal, 41(5), 347-354. doi: 10.1007/s10643-012-0572-3 
Preisler, G. M. (1995). The development of communication in blind and in deaf infantssimilarities and differences. Child: Care, Health and Development, 21(2), 79-110. doi: 10.1111/j.1365-2214.1995.tb00412.x

Pring, L. (1984). A comparison of the word recognition processes of blind and sighted children. Child Development, 55(5), 1865-1877. doi: 10.2307/1129933

Pring, L. (1994). Touch and go: Learning to read Braille. Reading Research Quarterly, 29(1), 67-74. doi: $10.2307 / 747738$

Pring, L. (2005). Autism and blindness: Building on the sum of their parts. London, UK: Whurr Publishers.

Ramani, G. B., \& Siegler, R. S. (2008). Promoting broad and stable improvements in low Dincome children's numerical knowledge through playing number board games. Child Development, 79(2), 375-394. doi: 10.1111/j.14678624.2007.01131.x

Ramey, C. T., \& Ramey, S. L. (2004). Early learning and school readiness: Can early intervention make a difference? Merrill-Palmer Quarterly, 50(4), 471-491.

Raver, C. C., \& Knitzer, J. (2002). Ready to enter: What research tells policymakers about strategies to promote social and emotional school readiness among threeand four-year-old children. New York, NY: National Center for Children in Poverty.

Repetto, J. B., \& Correa, V. I. (1996). Expanding views on transition. Exceptional Children, 62(6), 551-563. doi: 10.1177/001440299606200606 
Rettig, M. (1994). The play of young children with visual impairments: Characteristics and interventions. Journal of Visual Impairment \& Blindness, 88(5), 410-420.

Rex, E. J., Koenig, A. J., Wormsley, D. P., \& Baker, R. L. (1995). Foundations of braille literacy. New York, NY: American Foundation for the Blind.

Reynell, J. (1978). Developmental patterns of visually handicapped children. Child Care, Health and Development, 4(5), 291-303. doi: 10.1111/j.13652214.1978.tb00088.x

Richards, L., \& Morse, J.M. (2007). Readme first for a user's guide to qualitative methods ( $2^{\text {nd }}$ ed.). Thousand Oaks, CA: Sage.

Rimm-Kaufman, S. (2004). School transition and school readiness: An outcome of early childhood development. In R. Tremblay, R. Barr, R. Peters, (Eds.), Encyclopedia on Early Childhood Development (pp. 1-7). Montreal, Quebec: Centre of Excellence for Early Childhood Development.

Rimm-Kaufman, S. E., Early, D. M., Cox, M. J., Saluja, G., Pianta, R. C., Bradley, R. H., \& Payne, C. (2002). Early behavioral attributes and teachers' sensitivity as predictors of competent behavior in the kindergarten classroom. Journal of Applied Developmental Psychology, 23(4), 451-470. doi:10.1016/S01933973(02)00128-4

Rimm-Kaufman, S. E., \& Pianta, R. C. (2000). An ecological perspective on the transition to kindergarten: A theoretical framework to guide empirical research. Journal of Applied Developmental Psychology, 21(5), 491-511. doi:10.1016/S0193-3973(00)00051-4 
Rimm-Kaufman, S. E., Pianta, R. C., \& Cox, M. J. (2000). Teachers' judgments of problems in the transition to kindergarten. Early Childhood Research Quarterly, 15(2), 147-166. doi:10.1016/S0885-2006(00)00049-1

Roberts, R. N., Akers, A. L., \& Behl, D. D. (1996). Family-level service coordination within home visiting programs. Topics in Early Childhood Special Education, 16(3), 279-301. doi: 10.1177/027112149601600303

Rogers, S. J., \& Puchalski, C. B. (1984). Development of symbolic play in visually impaired young children. Topics in Early Childhood Special Education, 3(4), 5763. doi: $10.1177 / 027112148400300410$

Rogers, S. J., \& Puchalski, C. B. (1988). Development of object permanence in visually impaired infants. Journal of Visual Impairment \& Blindness, 87, 137-142.

Rous, B., Hemmeter, M. L., \& Schuster, J. (1994) Sequenced transition to education in the public schools: A systems approach to transition planning. Topics in Early Childhood Special Education, 14(3), 374-393.

doi: $10.1177 / 027112149401400307$

Sabol, T. J., \& Pianta, R. C. (2012a). Patterns of school readiness forecast achievement and socioemotional development at the end of elementary school. Child Development, 83(1), 282-299. doi: 10.1111/j.1467-8624.2011.01678.x

Sabol, T. J., \& Pianta, R. C. (2012b). Recent trends in research on teacher-child relationships. Attachment \& Human Development, 14(3), 213-231. doi: $10.1080 / 14616734.2012 .672262$ 
Sacks, S., Kekelis, L., \& Gaylord-Ross, R. (Eds.). (1992). The development of social skills by blind and visually impaired students: Exploratory studies and strategies. New York, NY: American Foundation for the Blind.

Saldaña, J. (2013). The coding manual for qualitative researchers ( $2^{\text {nd }}$ ed.). Thousand Oaks, CA: Sage.

Sandelowski, M. (2000). Focus on research methods: Whatever happened to qualitative description? Research in Nursing and Health, 23(4), 334-340. doi: 10.1002/1098240X(200008)23:4<334::AID-NUR9>3.0.CO;2-G

Sapp, W., \& Hatlen, P. (2010). The expanded core curriculum: Where we have been, where we are going, and how we can get there. Journal of Visual Impairment \& Blindness, 104(6), 338-348.

Schneekloth, L. H. (1989). Play environments for visually impaired children. Journal of Visual Impairment \& Blindness, 83(4), 196-201.

Schulting, A. B., Malone, P., \& Dodge, K. A. (2005). The effect of school-based kindergarten transition policies and practices on child academic outcomes. Developmental Psychology, 41(6), 860-871. doi: 10.1037/0012-1649.41.6.860

Scott-Little, C., Kagan, S., \& Frelow, V. (2006). Conceptualization of readiness and the content of early learning standards: The intersection of policy and research? Early Childhood Research Quarterly, 21(2), 153-173.

doi:10.1016/j.ecresq.2006.04.003 
Scott-Little, C., Lesko, J., Martella, J., \& Milburn, P. (2007). Early learning standards: Results from a national survey to document trends in state-level policies and practices. Early Childhood Research and Practice, 9(1), 1-23.

Scott-Little, C., \& Maxwell, K. (2000). School readiness in North Carolina: Strategies for defining, measuring, and promoting success for all children. Report of the Ready for School Goal Team. Greensboro, NC: Serve.

Sénéchal, M., LeFevre, J. A., Smith-Chant, B. L., \& Colton, K. V. (2001). On refining theoretical models of emergent literacy the role of empirical evidence. Journal of School Psychology, 39(5), 439-460. doi:10.1016/S0022-4405(01)00081-4

Seymour, P. H., \& Elder, L. (1986). Beginning reading without phonology. Cognitive Neuropsychology, 3(1), 1-36. doi: 10.1080/02643298608252668

Shaul, S., \& Schwartz, M. (2014). The role of the executive functions in school readiness among preschool-age children. Reading and Writing, 27(4), 749-768. doi: $10.1007 / \mathrm{s} 11145-013-9470-3$

Shore, R. (1998). Ready schools: A report of the goal 1 ready schools resource group. Washington, DC: The National Education Goals Panel.

Skiba, R. J., Simmons, A. B., Ritter, S., Gibb, A. C., Rausch, M. K., Cuadrado, J., \& Chung, C. G. (2008). Achieving equity in special education: History, status, and current challenges. Exceptional Children, 74(3), 264-288.

Smith, M. L., \& Shepard, L. A. (1988). Kindergarten readiness and retention: A qualitative study of teachers' beliefs and practices. American Educational Research Journal, 25, 307-333. doi: 10.3102/00028312025003307 
Snowling, M., \& Frith, U. (1981). The role of sound, shape and orthographic cues in early reading. British Journal of Psychology, 72(1), 83-87. doi: 10.1111/j.20448295.1981.tb02164.x

Sonksen, P.M., Levitt, S.L. \& Kitzinger, M. (1984) Identification of constraints acting on motor development in young visually disabled children and principles of remediation. Child: Care, Health and Development, 10, 273-86. doi: 10.1111/j.1365-2214.1984.tb00186.x

Special education provided by state through local, county or regional program, Oregon Legislative Counsel Committee, Chapter 343 Special Education and Other Specialized Services ORS 343.236 (1985).

Squires, J., Bricker, D., \& Twombly, E. (2002). The ASQ: SE user's guide: For the Ages \& Stages Questionnaires: Social-emotional. Baltimore, MD: Paul H Brookes.

Stauder, J., Molenaar, P., \& van der Molen, M. (1993). Scalp topography of eventrelated brain potentials and cognitive transition during childhood. Child Development, 64, 749-788. doi: 10.1111/j.1467-8624.1993.tb02942.x

Steinman, B. A., LeJeune, B. J., \& Kimbrough, B. T. (2006). Developmental stages of reading processes in children who are blind and sighted. Journal of Visual Impairment \& Blindness, 100(1), 36-46.

Stone, J. (1988). Can't I finish the story? Teaching braille to infants. British Journal of Visual Impairment, 6(2), 51-53. doi: 10.1177/026461968800600203 
Stormont, M., Beckner, R., Mitchell, B., \& Richter, M. (2005). Supporting successful transit on to kindergarten: General challenges and specific implications for students with problem behaviors. Psychology in the Schools, 42(8), 765-778. doi: $10.1002 /$ pits.20111

Stratton, J. M. (1996). Emergent literacy: A new perspective. Journal of Visual Impairment and Blindness, 90, 177-183.

Stratton, J. M., \& Wright, S. (1991). On the way to literacy: Early experiences for young visually impaired children. RE: view, 23(2), 55-62.

Stuart, M., Lieberman, L., \& Hand, K., (2006). Beliefs about physical activity among children who are visually impaired and their parents. Journal of Visual Impairment \& Blindness (JVIB), 100(4), 223-224.

Swenson, A. M. (1988). Using an integrated literacy curriculum with beginning braille readers. Journal of Visual Impairment and Blindness, 82(8), 336-338.

Swenson, A. M. (1999). Beginning with braille firsthand experiences with a balanced approach. New York, NY: AFB Press.

Tait, P.E. (1972). Behavior of young blind children in a controlled play situation. Perceptual and Motor Skills, 34, 963-969.

Thatcher, R.W. (1994). Cyclic cortical reorganization: Origins of human cognitive development. In G. Dawson \& K. W. Fischer (Eds.), Human behavior and the developing brain (pp. 233-266). New York, NY: Guilford Press.

Toussaint, K. A., \& Tiger, J. H. (2010). Teaching early braille literacy skills within a stimulus equivalence paradigm to children with degenerative visual 
impairments. Journal of Applied Behavior Analysis, 43(2), 181-194. doi:

10.1901/jaba.2010.43-181

Troster, H., \& Brambring, M. (1992). Early social $\square$ emotional development in blind infants. Child: Care, Health and Development, 18(4), 207-227. doi:

10.1111/j.1365-2214.1992.tb00355.x

Urwin, C. (1984a). Communication in infancy and the emergence of language in blind children. In R. L. Schiefelbusch \& J. Pickar (Eds.), The acquisition of communicative competence (pp. 479-524). Baltimore, MD: University Park Press.

Urwin, C. (1984b). Language for absent things: Learning from visually handicapped children. Topics in Language Disorders, 4(4), 24-37.

U.S. Department of Education. (2004). Building the legacy: IDEA 2004. Retrieved from http://idea.ed.gov

U.S. Department of Health and Human Services (USDHHS), Administration for Children \& Families, Office of Head Start. (2015). The Head Start Early Learning Outcomes Framework. (Publication No. HHSP233201000415G). Retrieved from http://eclkc.ohs.acf.hhs.gov/hslc/hs/sr/approach/elof

U.S. Department of Education, National Center for Education Statistics. (2015). Digest of Education Statistics, 2013 (NCES 2015-011).

Vogt, W. P., Gardner, D. C., \& Haeffele, L. M. (2012). When to use what research design. New York, NY: Guilford Press. 
Warren, D. (1984). Blindness and early childhood development. New York, NY: American Foundation for the Blind.

Warren, D. H., \& Hatton, D. D. (2003). Cognitive development in children with visual impairments. In I. Rapin \& S. Segalowitz (Eds.), Handbook of neuropsychology, 8 (Part II): Child neuropsychology (2nd ed., pp. 439-459). New York, NY: Elsevier.

Weiland, C., \& Yoshikawa, H. (2013). Impacts of a prekindergarten program on children's mathematics, language, literacy, executive function, and emotional skills. Child Development, 84(6), 2112-2130. doi: 10.1111/cdev.12099

Wesley, P. W., \& Buysse, V. (2003). Making meaning of school readiness in schools and communities. Early Childhood Research Quarterly, 18, 351-375. doi:10.1016/S0885-2006(03)00044-9

West, J., Jausken, E. G., \& Collins, M. (1993). Readiness for kindergarten: Parent and teacher beliefs. Washington, DC: U.S. Department of Education, Office of Research and Improvement (NCES 93257).

Wilde, A. (2009). God, money and politics: English attitudes to blindness and touch from the enlightenment to integration (review). Journal of Literary \& Cultural Disability Studies, 3(2), 212-214. doi: http://dx.doi.org/10.1353/jlc.0.0014

Wolfgang, C. H., Stannard, L. L., \& Jones, I. (2001). Block play performance among preschoolers as a predictor of later school achievement in mathematics. Journal of Research in Childhood Education, 15(2), 173-180. doi:

$10.1080 / 02568540109594958$ 
Workman, E., Griffith, M., \& Atchison, B. (2014). State pre-k funding-2013-14 fiscal year. Denver, CO: Education Commission of the States. Retrieved from http://www.ecs.org/clearinghouse/ 01/10/28/11034.pdf

Wormsley, D., \& D'Andrea, F. (Eds.). (1997). Instructional strategies for braille literacy. New York, NY: American Foundation for the Blind.

Xue, Y., \& Meisels, S. J. (2004). Early literacy instruction and learning in kindergarten: Evidence from the early childhood longitudinal study—-kindergarten class of 1998-1999. American Educational Research Journal, 41(1), 191-229. doi: $10.3102 / 00028312041001$

Yell, M. L., Rogers, D., \& Rogers, E. L. (1998). The legal history of special education what a long, strange trip it's been!. Remedial and Special Education, 19(4), 219228. 


\section{Appendix A}

\section{Request for Documents}

I am completing research as a doctoral student at Portland State University (PSU). The purpose of this research is to describe and explain how Teachers of the Visually Impaired (TVI) and primary caregivers construct the idea of school readiness for children who are blind and use braille as their primary learning medium. My intention is to identify current understanding and constructs and themes of school readiness that are reflected in Individual Family Service Plans (IFSP) and Individual Education Plans (IEP) for children who are blind and use braille as their primary medium. Themes identified by a document review will be verified through semi-structured interviews with TVIs and parents of children who are blind. Children who are blind are unique and have unique educational needs, these findings will provide a foundation to examine the type and intensity of services students receiving in early childhood special education (ECSE) and as they transition to kindergarten. It will potentially provide insight into recommendations from teachers in the field regarding recommendations and best practices for children who are blind entering kindergarten.

Criteria for inclusion in the study are students currently in or entering first grade or later and identified as using braille as their primary medium while in early childhood special education services. I am asking for you to send me copies of IFSP and IEP documents from ages 3-5 as well as the entering and exit IEP during the kindergarten year. Prior to sending me the document, please redact all identifying information relating to the student or professionals involved.

I am including a self-addressed stamped envelope for your convenience. If you have any questions, please contact me at Lisa.mcconachie@gmail.com or 503-475-9537.

Sincerely,

Lisa McConachie 


\section{Appendix B}

\section{Teacher of the Visually Impaired Consent Form}

You are invited to participate in a study conducted by Lisa McConachie, doctoral student from Portland State University (PSU). The purpose of this research is to describe and explain how Teachers of the Visually Impaired (TVI) and primary caregivers construct the idea of school readiness for children who are blind and use braille as their primary learning medium. My intention is to identify current understanding and constructs of school readiness from your perspective as a teacher of the visually impaired.

Understanding current notions of school readiness by interviewing key participants will allow a comparison of school readiness between children who are blind and those children who are sighted. Children who are blind are unique and have unique educational need, these findings will provide a foundation to examine the type and intensity of services students are currently receiving in early childhood special education (ECSE). It will potentially provide insight into recommendations from teachers in the field regarding recommendations and best practices for children who are blind entering kindergarten.

Your participation in the research will allow me to identify themes of the current understanding of school readiness from the perspective of the TVI. Your responses will also help highlight the current trends and identify possible recommendations for instructional changes for children who are blind and use braille as their primary learning medium.

If you agree to participate, you will be asked to participate in an interview with me, either in person or by phone. The interview will be approximately 30-60 minutes and will be audio-taped. Themes and conclusions resulting from the interview will be shared with you to verify accuracy.

You were selected as a participant for this study based on your position as a teacher of the visually impaired in Oregon. Participation is completely voluntary and all information will be confidential. No personal identifying information will be included; participants are from all regions in Oregon further reducing the risk to you as a professional. Information and data collected will be kept in a locked file cabinet and stored in the home office of Lisa McConachie for a period of three years. You do not have to take part in this study; it will not affect your relationship with your regional program. You may withdraw your participation at any time.

If you have concerns or problems about participating in this study or your rights as a research subject, please contact the Human Subjects Research Review Committee, Office of Research and Strategic Partnerships, Market Center Building, 1600 SW $4^{\text {th }}$, Portland State University, (503) 725-3423. If you have questions about the research itself, please contact Lisa McConachie, lisa.mcconachie@gmail.com. The researcher will provide you with a copy of this form for your records.

If you agree to participate, please return this form signed and marked "I agree" to Lisa McConachie at lisa.mcconachie@gmail.com or 2480 SW Timberline Drive, Portland, OR 97225. I will contact you within one week to schedule an interview at your convenience.

Your mark in the checkbox "I agree" below indicated that you have read and understand the above information and agree to take part in this study.

I agree Signature

Thank you for our consideration of participation.

Sincerely, Lisa McConachie 


\section{Appendix C}

\section{Primary Caregiver Consent Form}

You are invited to participate in a study conducted by Lisa McConachie, doctoral student from Portland State University (PSU). The purpose of this research is to describe and explain how Teachers of the Visually Impaired (TVI) and primary caregivers construct the idea of school readiness for children who are blind and use braille as their primary learning medium. My intention is to identify current understanding and constructs of school readiness from your perspective as a primary caregiver of a child who is blind and uses braille as their primary learning medium. Understanding current notions of school readiness by interviewing key participants will allow a comparison of school readiness between children who are blind and those children who are sighted. Children who are blind are unique and have unique educational needs, these findings will provide a foundation to examine the type and intensity of services students are currently receiving in early childhood special education (ECSE). It will potentially provide insight into recommendations from teachers in the field regarding recommendations and best practices for children who are blind entering kindergarten.

Your participation in the research will allow me to identify themes of the current understanding of school readiness from the perspective of primary caregivers of children who are blind. Your responses will also help highlight the current trends and identify possible recommendations for instructional changes for children who are blind and use braille as their primary learning medium.

If you agree to participate, you will be asked to participate in an interview with me, either in person or by phone. The interview will be approximately 30-60 minutes and will be audio-taped. Themes and conclusions resulting from the interview will be shared with you to verify accuracy.

Your regional program TVI sending you this notice selected you for this opportunity as a participant. Participation is completely voluntary and all information will be confidential. No personal or student identifying information will be included, participants are from all regions in Oregon further reducing the risk to you as a primary caregiver. Information and data collected will be kept in a locked file cabinet and stored in the home office of Lisa McConachie for a period of three years. You do not have to take part in this study; it will not affect your relationship with your regional program. You may withdraw your participation at any time.

If you have concerns or problems about participating in this study or your rights as a research subject, please contact the Human Subjects Research Review Committee, Office of Research and Strategic Partnerships, Market Center Building, 1600 SW $4^{\text {th }}$, Portland State University, (503) 725-3423. If you have questions about the research itself, please contact Lisa McConachie, lisa.mcconachie@gmail.com. The researcher will provide you with a copy of this form for your records.

If you agree to participate, please return this form signed and marked "I agree" to Lisa McConachie at lisa.mcconachie@gmail.com or 2480 SW Timberline Drive, Portland, OR 97225. I will contact you within one week to schedule an interview at your convenience.

Your mark in the checkbox "I agree" below indicated that you have read and understand the above information and agree to take part in this study. I agree Signature

Thank you for our consideration of participation.

Sincerely, Lisa McConachie 


\section{Appendix D}

\section{Sample Questions for Teacher of the Visually Impaired}

1. What is your understanding of the term "kindergarten readiness"?

2. What skills do you see as supporting a successful transition for a child who is blind to a typical kindergarten classroom?

3. What is the current focus of instruction for children who are blind in preschool to prepare them for kindergarten?

4. What are the barriers for a child who is blind attending a typical kindergarten?

5. How are children who are blind adequately prepared to participate in kindergarten considering the whole child?

6. How do you involve families in preparing their child for kindergarten?

7. If there were no caseload constraints, what would you see as the ideal service level in preschool for a child who reads braille? Daily? How much time? 


\section{Appendix E}

\section{Sample Questions for Primary Caregiver}

1. What was the focus of instruction for your child in preschool to prepare them for kindergarten? Did you receive instructional support in your home?

2. What were the successes and barriers for a child attending a typical kindergarten?

3. Please describe how your child was prepared to participate in kindergarten considering the whole child including peers, general education teachers, teachers of the visually impaired, you as care providers?

4. How comfortable were you engaging in pre-academic activities with your child considering the use of braille? Please talk about the reading environment in your home; did you have a nightly reading time, play family games?

5. Describe the services and education your child received during preschool and transitioning into kindergarten. What was your relationship with the teacher of the visually impaired? What was your relationship with the preschool/kindergarten teacher? 


\section{Appendix F}

\section{Student Data Analysis}

\begin{tabular}{|c|c|c|c|c|c|}
\hline Preschool 1 & Preschool 2 & Incoming K & Outgoing K & $\begin{array}{l}\text { HS } \\
\text { Domain/Sub } \\
\text { Domain }\end{array}$ & ECC \\
\hline $\begin{array}{l}\text { Use braille } \\
\text { writer to make } \\
\text { dots on a page, } \\
\text { insert paper, } \\
\text { identify parts } \\
\text { of a page. }\end{array}$ & & $\begin{array}{l}\text { Write in } \\
\text { braille the } \\
\text { letters of her } \\
\text { name. }\end{array}$ & $\begin{array}{l}\text { Read \& write } \\
\text { braille alphabet. }\end{array}$ & $\begin{array}{l}\text { Literacy: Print } \\
\text { \& alphabet } \\
\text { knowledge } \\
\text { Literacy: } \\
\text { Writing }\end{array}$ & $\begin{array}{l}\text { Compensatory/ } \\
\text { access skills }\end{array}$ \\
\hline \multirow[t]{3}{*}{$\begin{array}{l}\text { Touch \& feel, } \\
\text { match textures, } \\
\text { track lines of } \\
\text { braille, } \\
\text { recognize her } \\
\text { name in braille, } \\
\text { tactually search } \\
\text { for items. }\end{array}$} & $\begin{array}{l}\text { Track a } \\
\text { variety of } \\
\text { lines of } \\
\text { braille. } \\
\text { Recognize the } \\
\text { letters in her } \\
\text { name } \\
\text { individually \& } \\
\text { her whole } \\
\text { name in } \\
\text { braille. }\end{array}$ & $\begin{array}{l}\text { Track a } \\
\text { variety of } \\
\text { lines of } \\
\text { braille. } \\
\text { Recognize the } \\
\text { letters in her } \\
\text { name } \\
\text { individually \& } \\
\text { her whole } \\
\text { name in } \\
\text { braille. }\end{array}$ & \multirow[t]{3}{*}{$\begin{array}{l}\text { Read \& write } \\
50 \text { sight words } \\
\text { from grade } 1 \\
\text { curriculum in } \\
\text { braille format. }\end{array}$} & $\begin{array}{l}\text { Literacy: Print } \\
\text { \& alphabet } \\
\text { knowledge } \\
\text { Literacy: } \\
\text { Writing }\end{array}$ & $\begin{array}{l}\text { Compensatory/ } \\
\text { access skills }\end{array}$ \\
\hline & $\begin{array}{l}\text { Trace letters } \\
\text { in her name } \\
\text { (print) }\end{array}$ & & & $\begin{array}{l}\text { Literacy: Print } \\
\text { \& alphabet } \\
\text { knowledge }\end{array}$ & $\begin{array}{l}\text { Compensatory/ } \\
\text { access skills }\end{array}$ \\
\hline & & & & $\begin{array}{l}\text { Literacy: } \\
\text { Writing }\end{array}$ & \\
\hline
\end{tabular}




\begin{tabular}{|c|c|c|c|c|c|}
\hline Preschool 1 & Preschool 2 & Incoming $\mathrm{K}$ & Outgoing K & $\begin{array}{l}\text { HS } \\
\text { Domain/Sub } \\
\text { Domain }\end{array}$ & ECC \\
\hline & & & $\begin{array}{l}\text { Large print: } \\
\text { increase } \\
\text { reading \& } \\
\text { writing } \\
\text { readiness in } \\
\text { area of } \\
\text { phonemics \& } \\
\text { be able to } \\
\text { recognize/write } \\
\text { all upper \& } \\
\text { lower case } \\
\text { letter with } \\
\text { corresponding } \\
\text { sounds \& read } \\
1^{\text {st }} \text { grade level } \\
\text { sight words. }\end{array}$ & $\begin{array}{l}\text { Literacy: } \\
\text { Phonological } \\
\text { awareness } \\
\text { Literacy: } \\
\text { Writing }\end{array}$ & $\begin{array}{l}\text { Compensatory/ } \\
\text { access skills }\end{array}$ \\
\hline \multirow[t]{2}{*}{$\begin{array}{l}\text { Point to \& } \\
\text { count up to } 15 \\
\text { objects. }\end{array}$} & $\begin{array}{l}\text { Count tactile } \\
\text { items up to } \\
12 .\end{array}$ & $\begin{array}{l}\text { Count tactile } \\
\text { items up to } 12\end{array}$ & & $\begin{array}{l}\text { Mathematics } \\
\text { Development: } \\
\text { Counting \& } \\
\text { Cardinality }\end{array}$ & $\begin{array}{l}\text { Compensatory/ } \\
\text { access skills }\end{array}$ \\
\hline & & & $\begin{array}{l}\text { Read \& write } \\
\text { one and two } \\
\text { digit numbers } \\
\text { in nemeth } \\
\text { braille. }\end{array}$ & $\begin{array}{l}\text { Mathematics } \\
\text { Development: } \\
\text { Counting \& } \\
\text { Cardinality }\end{array}$ & $\begin{array}{l}\text { Compensatory/ } \\
\text { access skills }\end{array}$ \\
\hline $\begin{array}{l}\text { Follow teacher } \\
\text { directions, } \\
\text { participate in } \\
\text { small \& large } \\
\text { group } \\
\text { activities; } \\
\text { follow class } \\
\text { routine } \\
\text { independently. }\end{array}$ & & & & $\begin{array}{l}\text { Approaches to } \\
\text { Learning: } \\
\text { Emotional \& } \\
\text { Behavioral } \\
\text { Self- } \\
\text { Regulation }\end{array}$ & $\begin{array}{l}\text { Compensatory/ } \\
\text { access skills }\end{array}$ \\
\hline
\end{tabular}




\begin{tabular}{|c|c|c|c|c|c|}
\hline Preschool 1 & Preschool 2 & Incoming $\mathrm{K}$ & Outgoing K & $\begin{array}{l}\text { HS } \\
\text { Domain/Sub } \\
\text { Domain }\end{array}$ & ECC \\
\hline \multirow[t]{3}{*}{$\begin{array}{l}\text { Interact more } \\
\text { frequently } \\
\text { w/other } \\
\text { children } \\
\text { including } \\
\text { maintaining } \\
\text { social } \\
\text { interactions } \\
\text { w/at least } 3 \\
\text { exchanges. }\end{array}$} & $\begin{array}{l}\text { Interact more } \\
\text { frequently } \\
\text { w/other } \\
\text { children } \\
\text { including } \\
\text { maintaining } \\
\text { social } \\
\text { interactions } \\
\text { w/at least } 3 \\
\text { exchanges. }\end{array}$ & $\begin{array}{l}\text { Interact with } \\
\text { peers } \\
\text { including } \\
\text { initiating \& } \\
\text { maintaining } \\
\text { social } \\
\text { interactions } \\
\text { w/at least } 3 \\
\text { exchanges. }\end{array}$ & & $\begin{array}{l}\text { Social \& } \\
\text { Emotional } \\
\text { Development: } \\
\text { Relationship } \\
\text { w/ other } \\
\text { children }\end{array}$ & $\begin{array}{l}\text { Social } \\
\text { Interaction } \\
\text { Skills }\end{array}$ \\
\hline & $\begin{array}{l}\text { Label } 6 \\
\text { emotions from } \\
\text { pictures; tell } \\
\text { adults how } \\
\text { she is feeling } \\
\text { and use self- } \\
\text { calming } \\
\text { techniques }\end{array}$ & $\begin{array}{l}\text { Label } 6 \\
\text { emotions from } \\
\text { pictures; tell } \\
\text { adults how } \\
\text { she is feeling } \\
\text { and use self- } \\
\text { calming } \\
\text { techniques }\end{array}$ & & $\begin{array}{l}\text { Social \& } \\
\text { Emotional } \\
\text { Development: } \\
\text { Emotional } \\
\text { Functioning }\end{array}$ & $\begin{array}{l}\text { Social } \\
\text { Interaction } \\
\text { Skills }\end{array}$ \\
\hline & $\begin{array}{l}\text { Use toilet } \\
\text { independently. }\end{array}$ & $\begin{array}{l}\text { Increase her } \\
\text { independence } \\
\text { in bathroom } \\
\text { routine. }\end{array}$ & $\begin{array}{l}\text { Learn to find } \\
\text { washroom, } \\
\text { locate toilet } \\
\text { paper, pull } \\
\text { from roll, flush } \\
\text { toilet \& orient } \\
\text { back to } \\
\text { classroom. } \\
\text { Increase visual } \\
\text { efficiency skills } \\
\text { using } \\
\text { magnifiers. }\end{array}$ & $\begin{array}{l}\text { Perceptual, } \\
\text { Motor, \& } \\
\text { Physical } \\
\text { Development: } \\
\text { Health, Safety, } \\
\text { \& Nutrition }\end{array}$ & $\begin{array}{l}\text { Independent } \\
\text { Living Skills }\end{array}$ \\
\hline
\end{tabular}




\begin{tabular}{|c|c|c|c|c|c|}
\hline Preschool 1 & Preschool 2 & Incoming K & Outgoing K & $\begin{array}{l}\text { HS } \\
\text { Domain/Sub } \\
\text { Domain }\end{array}$ & ECC \\
\hline $\begin{array}{l}\text { Develop fine } \\
\text { motor skills by } \\
\text { stringing } \\
\text { beads, snipping } \\
\text { w/scissors \& } \\
\text { scribble on } \\
\text { page. } \\
\text { Use a cane, } \\
\text { hold it in } \\
\text { correct position } \\
\text { in familiar \& } \\
\text { unfamiliar } \\
\text { environments. }\end{array}$ & $\begin{array}{l}\text { Hold, store, } \\
\text { and use cane } \\
\text { appropriately. } \\
\text { Detect drop } \\
\text { offs. }\end{array}$ & $\begin{array}{l}\text { Store cane in } \\
\text { consistent } \\
\text { place \& use } \\
\text { constant } \\
\text { contact } \\
\text { technique. } \\
\text { Locate } 5 \text { or } \\
\text { more } \\
\text { destinations in } \\
\text { school; } \\
\text { identify } 12 \\
\text { directional \& } \\
\text { positional } \\
\text { concepts. }\end{array}$ & $\begin{array}{l}\text { Increased } \\
\text { independence } \\
\text { in locating } \\
\text { specific } \\
\text { locations; use } \\
12 \text { directional } \\
\& \text { positional } \\
\text { concepts. }\end{array}$ & $\begin{array}{l}\text { Perceptual, } \\
\text { Motor, \& } \\
\text { Physical } \\
\text { Development: } \\
\text { Gross Motor }\end{array}$ & $\begin{array}{l}\text { Compensatory/ } \\
\text { access skills }\end{array}$ \\
\hline
\end{tabular}

